\title{
‡USGS
}

Prepared in cooperation with the Bureau of Economic Geology, The University of Texas at Austin

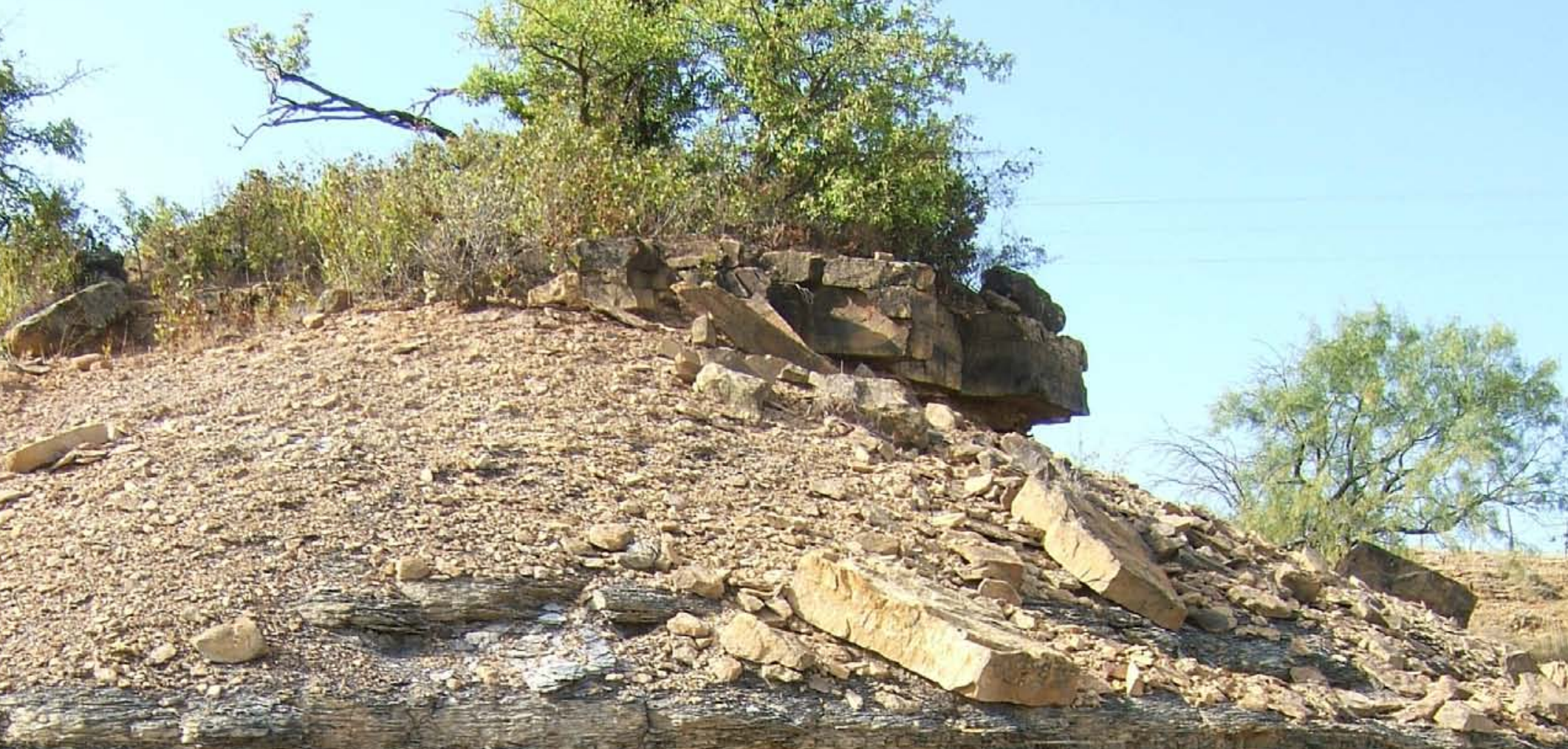

\section{Coal rank and stratigraphy of Pennsylvanian coal and coaly shale samples, Young Gounty, north-central Texas}

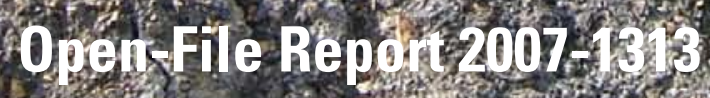

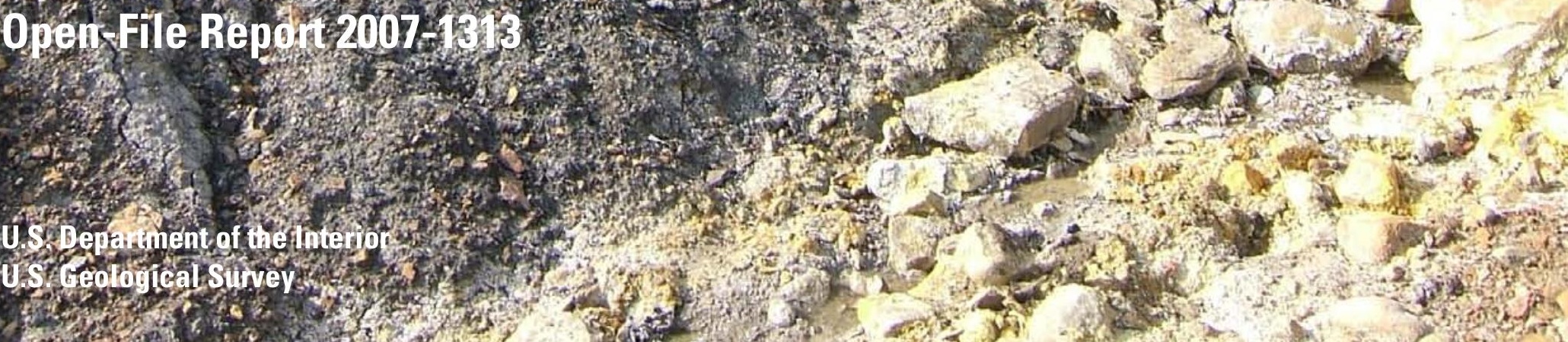




\title{
U.S. Department of the Interior
}

\author{
Dirk A. Kempthorne, Secretary
}

\section{U.S. Geological Survey}

Mark D. Myers, Director

\section{U.S. Geological Survey, Reston, Virginia 2006}

For product and ordering information:

World Wide Web: http://www.usgs.gov/pubprod

Telephone: 1-888-ASK-USGS

For more information on the USGS - the Federal source for science about the Earth, its natural and living resources, natural hazards, and the environment:

World Wide Web: http://www.usgs.gov

Telephone: 1-888-ASK-USGS

Suggested citation:

Guevara, E. H., Breton, C., and Hackley, P.C., 2007, Coal rank and stratigraphy of Pennsylvanian coal and coaly shale samples, Young County, north-central Texas: U.S. Geological Survey Open-File Report 20071313, 28 p., 1 plate, http://pubs.usgs.gov/of/2007/1313.

Any use of trade, product, or firm names is for descriptive purposes only and does not imply endorsement by the U.S. Government.

Although this report is in the public domain, permission must be secured from the individual copyright owners to reproduce any copyrighted material contained within this report. 


\section{Contents}

Abstract

Introduction

Objectives

Data

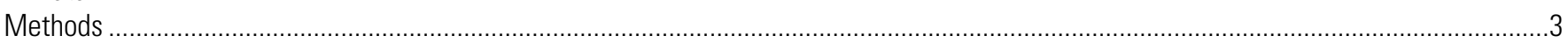

Results

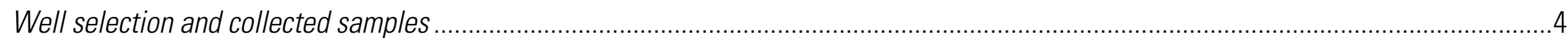

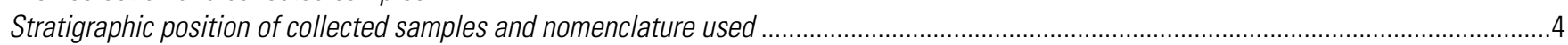

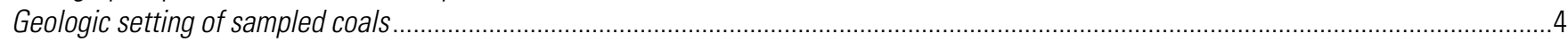

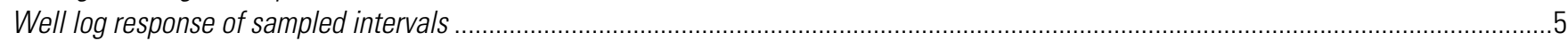

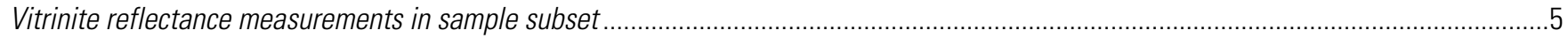

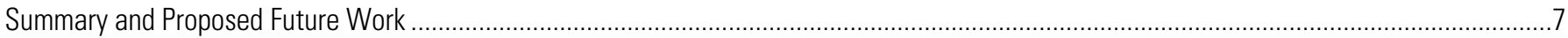

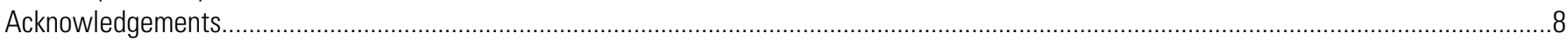

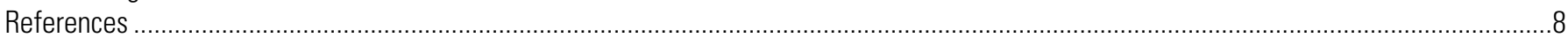

Table 1 .

Table 2

Table 3

Appendix: Coal and coaly shale in drill cuttings descriptions, Young County, north-central Texas.................................................................. 


\title{
Coal rank and stratigraphy of Pennsylvanian coal and coaly shale samples, Young County, north-central Texas
}

\author{
By Edgar H. Guevara', Caroline Breton', and Paul C. Hackley² \\ 'Bureau of Economic Geology, The University of Texas at Austin, Austin, TX 78713 \\ U.S. Geological Survey, MS 956 National Center, Reston, VA 20192
}

\begin{abstract}
Vitrinite reflectance $\left(\mathrm{R}_{0}\right)$ measurements were made to determine the rank of selected subsurface coal and coaly shale samples from Young County, north-central Texas, for the National Coal Resources Database System (NCRDS) State Cooperative Program conducted by the Bureau of Economic Geology at The University of Texas at Austin. This research is the continuation of a pilot study that began in adjacent Archer County, and forms part of a larger investigation of the coalbed methane resource potential of Pennsylvanian coals in north-central Texas.

A total of 57 samples of coal and coaly shale fragments were hand-picked from drill cuttings from depths of about 2,000 ft in five wells, and $R_{0}$ determinations were made on an initial 10-sample subset. Electric-log correlation of the sampled wells indicates that the collected samples represent coal and coaly shale layers in the Strawn (Pennsylvanian), Canyon (Pennsylvanian), and Cisco (Pennsylvanian-Permian) Groups.

Coal rank in the initial sample subset ranges from lignite $\left(\mathrm{R}_{0}=0.39\right)$, in a sample from the Cisco Group at a depth of 310 to $320 \mathrm{ft}$, to high volatile bituminous A coal $\left(\mathrm{R}_{0}=0.91\right)$ in a sample from the lower part of the Canyon Group at a depth of 2,030 to 2,040 ft.
\end{abstract}

\section{Introduction}

This report summarizes studies of the Texas Coal Sample Collection for Rank Determination project carried out by the Bureau of Economic Geology (BEG) at The University of Texas at Austin from April 2005 through February 2006. This work is part of the National
Coal Resources Database System (NCRDS) State Cooperative Program of the U.S. Geological Survey (USGS). Coal and coaly shale fragments were collected from drill cuttings from five wells in Young County (fig. 1 ), and vitrinite reflectance measurements $\left(R_{0}\right)$ were made on a subset of the samples. The stratigraphy containing the sampled intervals is summarized in figure 2. These studies are a continuation of a pilot program of subsurface coal sampling by the BEG that began in 2004 in adjacent Archer County (Guevara and Sakurai, 2005; fig. 1), and form part of a larger assessment of the coalbed methane resource potential of Pennsylvanian coals in north-central Texas.

The sampled wells are located north (downdip) of outcrops of Pennsylvanian bituminous coal (fig. 1) whose stratigraphic position, characteristics, and depositional setting were summarized in Mapel (1967), Brown and others (1972, 1987, 1990), Galloway and Brown (1972), Brown (1973), Erxleben (1973, 1974), Cleaves (1973, 1975), and Evans (1974). Data on the regional extent and properties of these coals were compiled by Kim (2003) in a geographic information system (GIS) database for the NCRDS program.

\section{Objectives}

The objectives of this investigation were to assess the rank of coals in the shallow subsurface of Young County (at depths of up to about 2,000 ft), and to estimate the stratigraphic position of the samples collected for the assessment.

Data

Five wells (Table 1) were selected from candidate wells identified in Brown and others (1987) on the basis of the availability of drill cuttings and electric logs on 


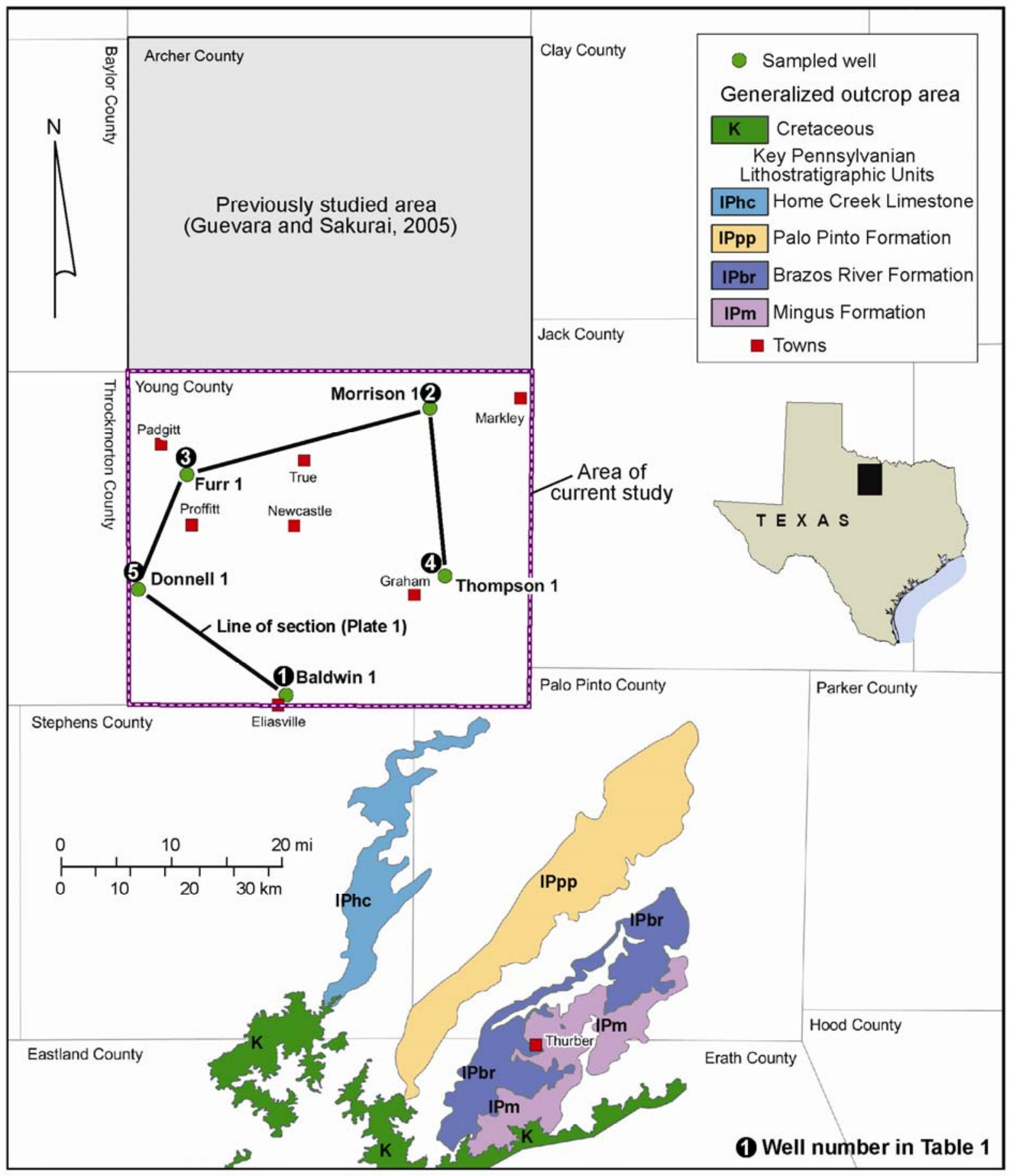

Figure 1. Index map of the study area showing locations of sampled wells and generalized outcrop area of key Pennsylvanian lithostratigraphic units. Young County lies north (downdip) of the outcrop area of north-central Texas bituminous coals. Outcrop area from Brown and others (1972). 


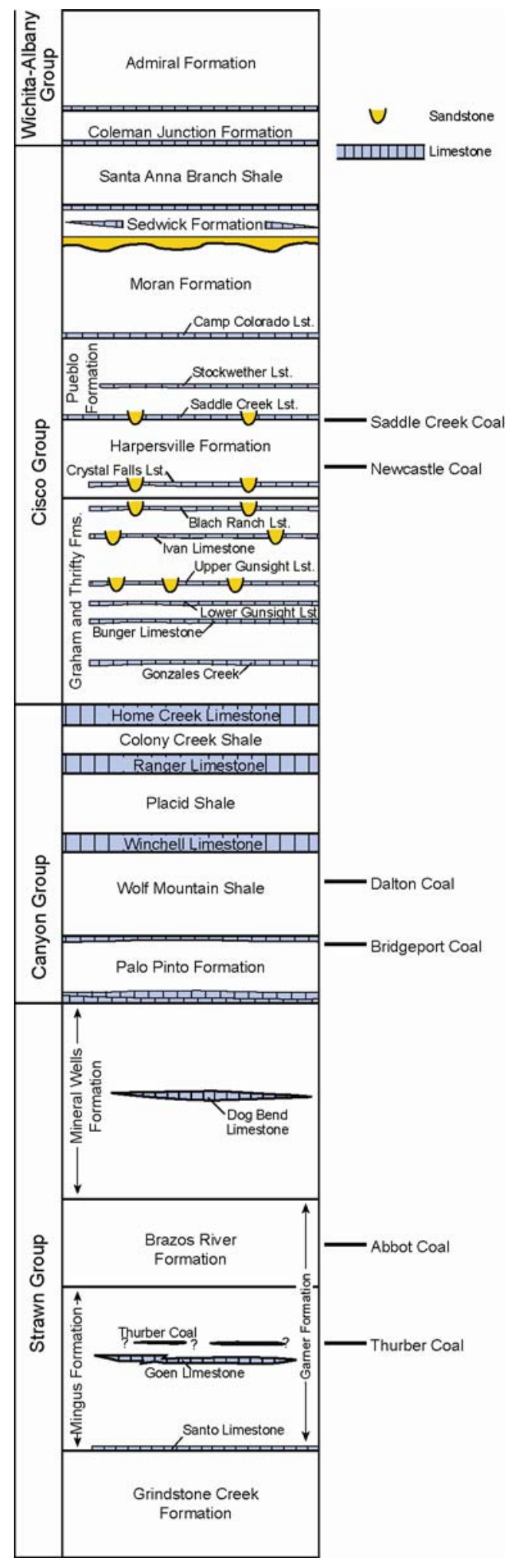

Figure 2. Generalized stratigraphic column of the Pennsylvanian in northcentral Texas illustrating the nomenclature used in this report and the approximate stratigraphic position of coal beds in Mapel (1967). Stratigraphic nomenclature and subdivisions are from Brown and others (1972). open-file at BEG. Logs used in the study depict spontaneous potential (SP) and resistivity curves at a vertical scale of 1 inch $=100 \mathrm{ft}$ and have handwritten stratigraphic annotations. These archival well logs were used in the studies of Wermund and Jenkins (1970) (L. F. Brown, Jr., personal communication), and in Brown and others (1987, 1990). Additionally, typewritten, narrative descriptions of drill cuttings from Young County, on open-file at BEG, were reviewed for reported coal occurrences.

\section{Methods}

The location of each sampled well was plotted on an ArcView* map of Young County, Texas. Neuralog software was used for log-curve digitization, and for displays of each well log. A well log cross section (Plate 1) was produced using Paradigm Geophysical's GEOLOG software.

Four main activities were carried out in the study: (1) well identification, screening, and selection for sampling; (2) collection of coal and coaly shale fragments from washed drill cuttings kept on open-file at BEG; (3) estimation of the stratigraphic position of collected samples; and (4) selection and analysis of an initial 10-sample subset to ascertain the range of $R_{0}$ values at depths of up to about 2,000 ft. $R_{0}$ measurements on the sample subset were provided by Humble Geochemical Services, Humble, Texas.

Table 1. Names and locations of sampled wells, Young County, north-central Texas. Drill cuttings and corresponding electric logs are on open-file at The University of Texas at Austin, Bureau of Economic Geology.

\begin{tabular}{|c|c|c|}
\hline $\begin{array}{l}\text { Well name } \\
\text { (Operator, Lease, Well No.) }\end{array}$ & $\begin{array}{l}\text { Derrick Floor } \\
\text { Elevation (ft) }\end{array}$ & Location \\
\hline $\begin{array}{l}\text { 1. Deep Rock Oil Corp., } \\
\text { C.R. Baldwin.Jr. No. } 1 *\end{array}$ & 1,129 & $\begin{array}{l}\text { TE\&L Co. Survey, } 1.400 \mathrm{ft} \\
\text { FSL of farm and on line of } \\
\text { section 1020-1019: } 1 \text { mi from } \\
\text { Eliasville. }\end{array}$ \\
\hline $\begin{array}{l}\text { 2. Kerlyn Oil Company. } \\
\text { Gillie-Morrison No.1 }\end{array}$ & 1.162 & $\begin{array}{l}\text { G\&BN Survey. sec. } 1.528 \mathrm{ft} \\
N \text { and } 825 \mathrm{ft} \text { Eof the SW } \\
\text { comer of the Davidson } \\
\text { survey Abstract } 84 \text {, which is the } \\
\text { SE } 1 / 4 \text { of sec. } 1 \text {. }\end{array}$ \\
\hline $\begin{array}{l}\text { 3. Lipscomb and De Lange. } \\
\text { Adam Furr No. } 1\end{array}$ & 1.161 & $\begin{array}{l}\text { TE\&L Co. survey, } 621 \mathrm{ft} \\
\text { FEL and } 333 \mathrm{ft} \text { FSL of } \\
\text { section } 338: 3 \mathrm{mi} \mathrm{SE} \text { of } \\
\text { Padgitt. }\end{array}$ \\
\hline $\begin{array}{l}\text { 4. Panhandle Refining } \mathrm{Co} . \\
\text { Eula Thompson No.1 }\end{array}$ & 1.115 & $\begin{array}{l}\text { TE\&L Co. survey, No. } 2692 \text {. } \\
764 \mathrm{ft} \text { FNEL and } 1,401 \mathrm{ft} \\
\text { FSEL of survey. }\end{array}$ \\
\hline $\begin{array}{l}\text { 5. Standard of Texas and } \\
\text { H. Buchanan. SR. Donnell No. } 1\end{array}$ & 1,246 & $\begin{array}{l}\text { TE\&L Co. survey, section } \\
2377,1,400 \mathrm{ft} \mathrm{FNL} \text { and } 467 \mathrm{ft} \\
\text { FWL }\end{array}$ \\
\hline
\end{tabular}

'Well 81 in Brown and others (1987), Plate XIX, cross section 4-4' 
Estimation of the stratigraphic position of samples was accomplished by correlating the electric log of one of the sampled wells (Baldwin No. 1; Plate 1) with the logs from the other sampled wells; the Baldwin 1 well was also used in Brown and others (1987; plate XIX, cross section 4-4', well 81). Additionally, general well information and depths of drill cuttings with narrative descriptions on open-file at BEG indicating occurrence of coal, black shale, or dark shale were compiled for 597 wells (see Appendix; coal occurrences highlighted).

\section{Results}

\section{Well selection and collected samples}

Fifty-seven samples of coal and coaly shale fragments (Table 2) were hand-picked from drill cuttings from mostly $<2,000 \mathrm{ft}$ depths in five wells for which drill cuttings and electric logs were on open-file at BEG. The coal and coaly shale fragments are minor components in the sampled drill cuttings and some of the collected samples were extremely small.

\section{Stratigraphic position of collected samples and nomenclature used}

Electric-log correlation of the sampled wells allowed estimation of the stratigraphic position of the collected samples. Lithostratigraphic subdivisions in Brown and others (1987) were used in the correlation. Table 3 records the tops of correlated lithostratigraphic units, and Plate 1 displays the stratigraphic position of collected samples with respect to the correlated units.

Well log correlation indicates that the collected samples represent coal and coaly shale layers forming part of (1) the Strawn Group (middle Pennsylvanian), probably at stratigraphic positions equivalent to Thurber and Abbot coalbeds in Mapel (1967); (2) the Canyon Group (Pennsylvanian), probably equivalent to Dalton and Bridgeport coalbeds in Mapel (1967); and (3) the Cisco Group (Pennsylvanian-Permian), probably at stratigraphic positions comparable to Newcastle and Saddle Creek coalbeds in Mapel (1967) (fig. 2).

Following the usage described by Brown (1959), units correlated in this study are lithostratigraphic. Boundaries of the Pennsylvanian Groups shown in Plate 1 are based on Brown and others (1972, 1987, 1990), with the top of the Strawn Group defined by the base of the Palo Pinto Formation (generalized outcrop area shown on fig. 1), the top of the Canyon Group defined by the top of the Home Creek Limestone (generalized outcrop area shown in fig. 1), and the top of the Cisco Group defined by the base of the Coleman Junction Formation (not shown in fig. 1). Therefore, coal occurrences in stratigraphic intervals younger than the
Table 2. Depth of coal and coaly shale samples collected in Young County, north-central Texas, and results of vitrinite reflectance analysis $\left(R_{0}\right)$ on an initial 10 -sample subset. $\mathrm{R}_{0}$ analysis by Humble Geochemical Services. All log depths are in $\mathrm{ft}$.

\begin{tabular}{|c|c|c|c|c|}
\hline $\begin{array}{l}\text { Sample ID } \\
\text { (well and depth in ti) }\end{array}$ & $R_{0}$ & n & s.d. & $\begin{array}{l}\text { Lithostratigraphic } \\
\text { Group }\end{array}$ \\
\hline $\begin{array}{l}\text { Baldwin No. } 1280-290 \\
\text { Baldwin No. } 1560-570 \\
\text { Baldwin No. } 1800-810 \\
\text { Baldwin No. } 1810-820 \\
\text { Baldwin No. } 1980-990 \\
\text { Baldwin No. } 11 \text { 1 } 1460-1470 \\
\text { Baldwin No. } 12750-2760 \\
\text { Baldwin No. } 2760-2770 \\
\text { Baldwin No. } 12770-2780\end{array}$ & 0.63 & 27 & 0.05 & $\begin{array}{l}\text { Cisco } \\
\text { Canyon } \\
\text { Canyon } \\
\text { Canyon } \\
\text { Canyon } \\
\text { Strawn } \\
\text { Strawn } \\
\text { Strawn } \\
\text { Strawn }\end{array}$ \\
\hline $\begin{array}{l}\text { Morrison No. } 1240-250 \\
\text { Marrison No. } 1310-320 \\
\text { Morrison No. } 11 \text { 1350-1360 } \\
\text { Marrison No.1 } 1650-1660 \\
\text { Morrison No.1 } 1730-1740 \\
\text { Morrison No.1 } 2030-2040\end{array}$ & $\begin{array}{l}0.39 \\
0.52\end{array}$ & $\begin{array}{l}30 \\
10\end{array}$ & $\begin{array}{l}0.06 \\
0.06\end{array}$ & $\begin{array}{l}\text { Cisco } \\
\text { Cisco } \\
\text { Canyon } \\
\text { Canyon } \\
\text { Canyon } \\
\text { Canyon }\end{array}$ \\
\hline 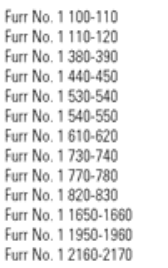 & $\begin{array}{c}\text { no vitrinite } \\
0.55\end{array}$ & 26 & 0.04 & $\begin{array}{l}\text { Cisco } \\
\text { Cisco } \\
\text { Cissco } \\
\text { Cisco } \\
\text { Cisco } \\
\text { Cisco } \\
\text { Cisso } \\
\text { Cisco } \\
\text { Cisco } \\
\text { Cisco } \\
\text { Caryon } \\
\text { Canyon } \\
\text { Canyon }\end{array}$ \\
\hline $\begin{array}{l}\text { Thompson No. } 1780-790 \\
\text { Thompson No. } 1820-830 \\
\text { Thompson No. } 1980-990\end{array}$ & 0.51 & 27 & 0.06 & $\begin{array}{l}\text { Canyon } \\
\text { Canyon } \\
\text { Canyon }\end{array}$ \\
\hline 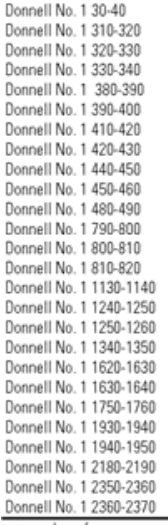 & no vitrinite & 31 & 0.08 & 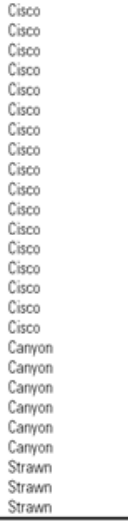 \\
\hline
\end{tabular}

Crystal Falls Limestone are assigned in this study to the Cisco Group (Pennsylvanian-Permian), not to the Wichita Group (Lower Permian), as they were in Mapel (1967). Conflicts in the stratigraphic nomenclature of Pennsylvanian-Lower Permian strata in north-central Texas largely result from issues of lithostratigraphy versus chronostratigraphy (Brown, 1959).

\section{Geologic setting of sampled coals}

North-central Texas coals have been interpreted to be allochthonous detrital accumulations occurring in interdeltaic embayments flanking delta systems (e.g., Galloway and Brown, 1972; Cleaves, 1973; Brown, 1973).

Erxleben $(1973,1974)$ interpreted coals in the Canyon Group as forming part of the Perrin Delta System, and, according to Brown (1973), coals in the Cisco Group represent delta-plain facies of elongate deltas having a vertical facies succession comparable 
Table 3. Pennsylvanian lithostratigraphy and tops of the units correlated on logs of the sampled wells, Young County, north-central Texas. Nomenclature according to Brown (1973), and Brown and others $(1987,1990)$. Log depths are in feet.

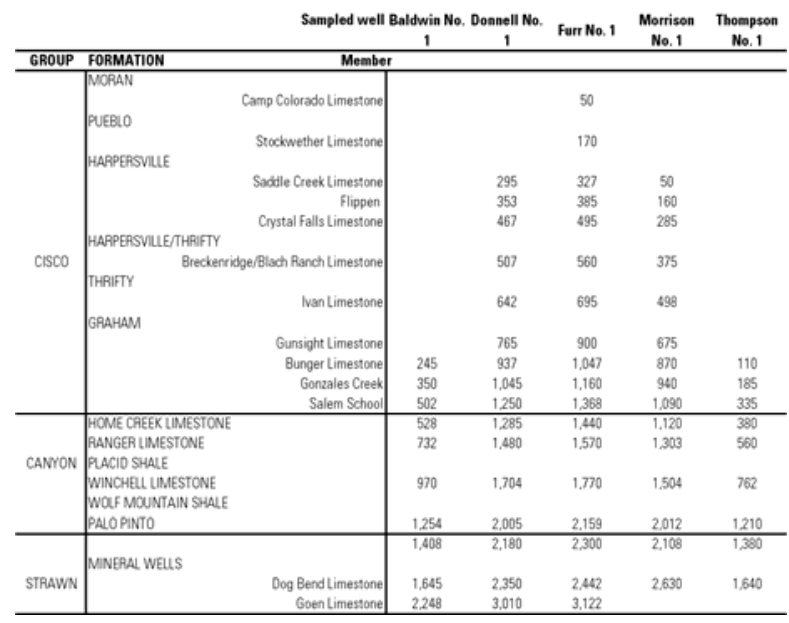

(although not in scale) to that of the modern Mississippi birdfoot delta.

The north-central Texas Pennsylvanian coals have been described as thin (<3 ft thick) (e.g., Mapel, 1967) and discontinuous (Galloway and Brown, 1972); the limited thickness and lateral extent interpreted to reflect the detrital origin and depositional setting. Drill-cutting descriptions from Young County (see Appendix) also suggest generally thin, laterally discontinuous coal occurrences at depths of up to 2,000 ft.

\section{Well log response of sampled intervals}

Electric logs used in the study did not allow unambiguous identification of the coaly intervals that were sampled-largely a consequence of the limited resolution of the vertical scale $(1$ inch $=100 \mathrm{ft})$ of the logs. Some of the sharp, thin, high-resistivity deflections observed in the Pennsylvanian section (Plate 1) may reflect limestone, coal, or closely-spaced coal and limestone beds.

The logs show relatively high resistivity, as expected from coal, at or very close to some of the intervals sampled-for example in the Morrison No. 1 (at depths of 240-250 ft, 310-320 ft, and 1,350-1,360 ft) and Baldwin No. 1 wells (at depths of 280-290 ft, 560-570 $\mathrm{ft}$, and 980-990 ft). Lack of high-resistivity readings on the log for some of the sampled intervals may result from thin coalbeds that are unresolved by the logs used.

Wide spacing of the sampled wells does not allow estimates of coal thickness or lateral continuity; however, the lack of thick high resistivity deflections suggests that the sampled coals probably are thin and comparable to those in outcrop. Use of porosity logs (e.g., density, sonic) and more closely-spaced wells would improve the identification and estimates of thickness and extent of coals in the subsurface of north-central Texas.

\section{Vitrinite reflectance measurements in sample subset}

An initial subset of 10 samples was selected to estimate the overall range of vitrinite reflectance values $\left(R_{0}\right)$ in samples from depths of up to about 2,000 ft. Humble Geochemical Services (HGS), Humble, Texas, performed the $\mathrm{R}_{\mathrm{o}}$ analyses.

$R_{o}$ values were obtained for eight samples; vitrinite was not present in two samples and $R_{0}$ values were not obtained for those samples (Table 2). Histograms of $R_{0}$ measurements obtained for each sample which contained vitrinite are shown in figures 3-10. The highest and lowest $\mathrm{R}_{\mathrm{o}}$ values were obtained in the Morrison No. 1 well, northeastern Young County $\left(\mathrm{R}_{\mathrm{o}}=0.39\right.$ at $310-320 \mathrm{ft}$ and $\mathrm{R}_{0}=0.91$ at $2,030-2,040 \mathrm{ft}$ ). $\mathrm{R}_{0}$ values $>0.60$ were determined for three samples, one from the Strawn Group in the Baldwin No. 1 well, and two from the Cisco Group; one in the Baldwin No. 1 well and the other in the Morrison No. 1 well (figs. 3-10 and Table 2).

HGS reported recycled nonindigenous vitrinite only in the Morrison No. 1 well (sample 1,350-1,360 ft; fig. 7 ). The $R_{0}=0.91$ value in the same well $(2,030-2,040 \mathrm{ft})$, however, may be anomalously high and should be reassessed.

The remaining samples are being prepared at USGS for additional vitrinite reflectance analysis and archiving.

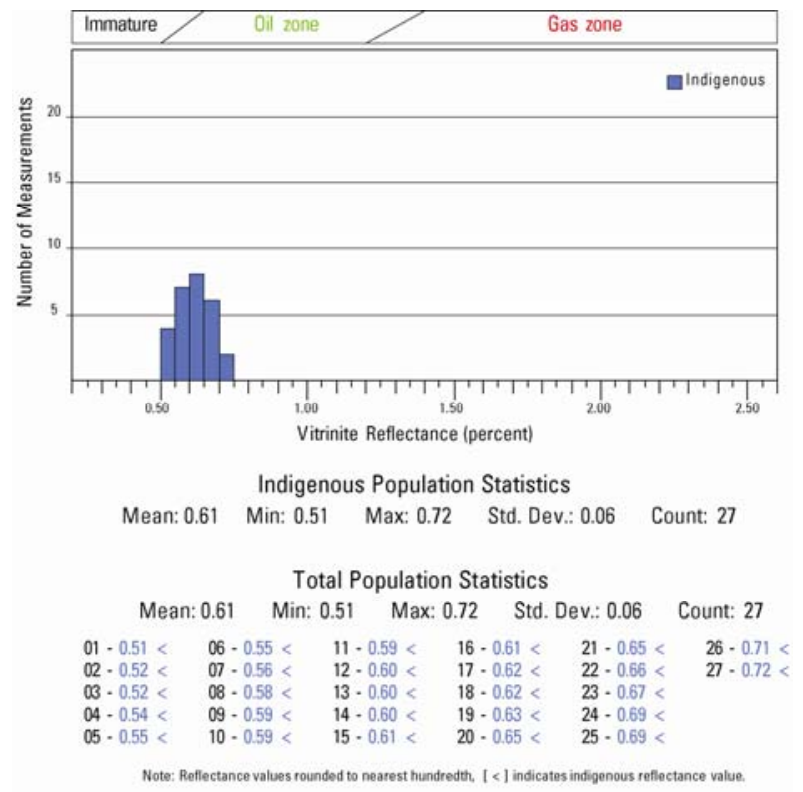

Figure 3. Histogram of $R_{0}$ measurements on drill cuttings from $560-570 \mathrm{ft}$ in the Deep Rock Corp., Baldwin No. 1 well, Young County, north-central Texas. $R_{0}=$ 0.61 based on 27 measurements. Measurements and histogram were provided by Humble Geochemical Services. 


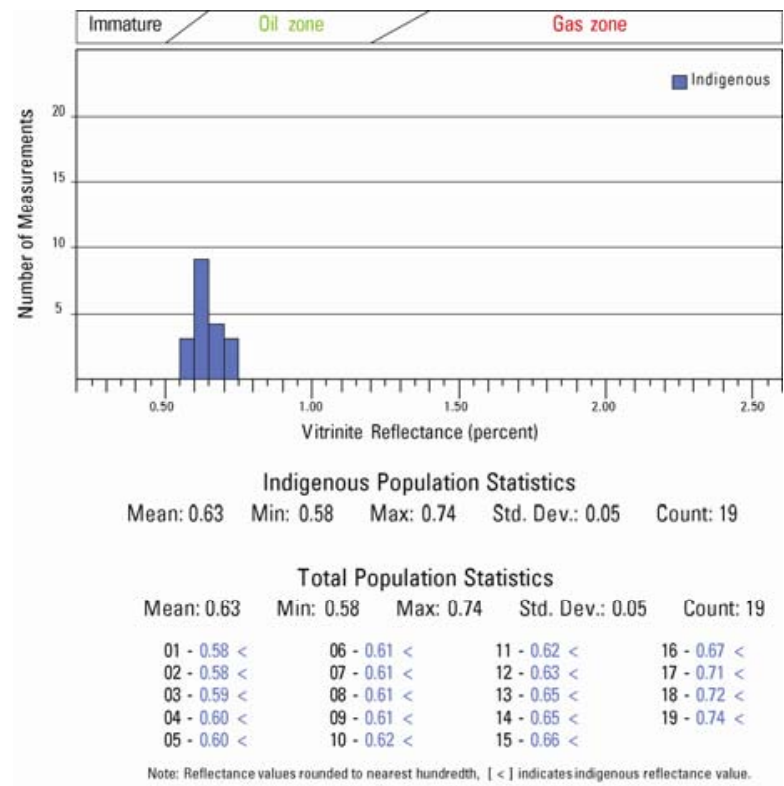

Figure 4. Histogram of $R_{0}$ measurements on drill cuttings from 1,460-1,470 ft in the Deep Rock Corp., Baldwin No. 1 well, Young County, north-central Texas. $\mathrm{R}_{0}=0.63$ based on 19 measurements. Measurements and histogram were provided by Humble Geochemical Services.

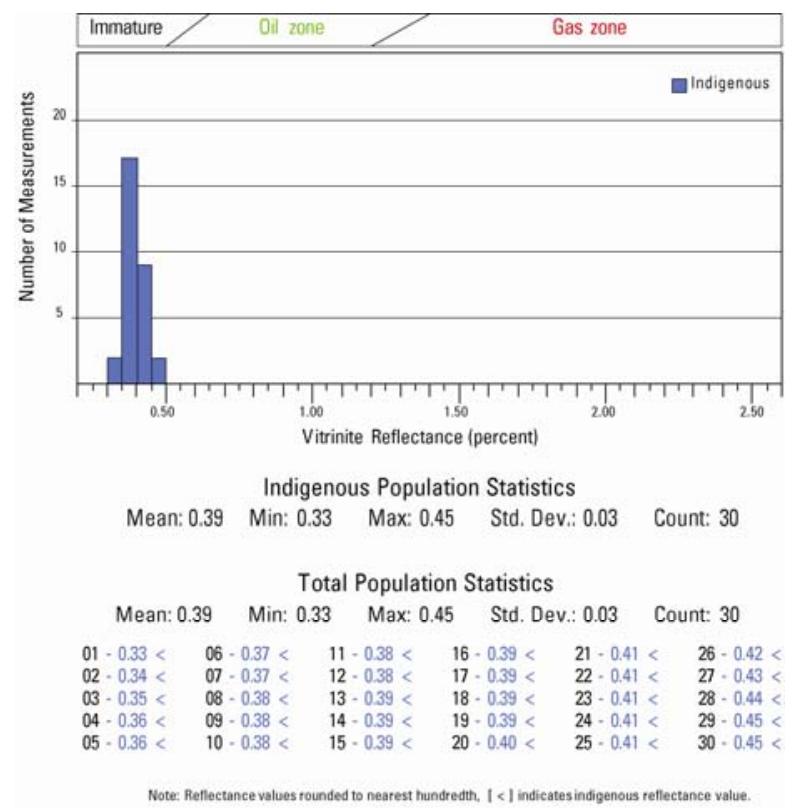

Figure 5. Histogram of $R_{0}$ measurements on drill cuttings from $310-320 \mathrm{ft}$ in the Kerlyn Oil Company, Morrison No. 1 well, Young County, north-central Texas. $R_{0}=0.39$ based on 30 measurements. Measurements and histogram were provided by Humble Geochemical Services.

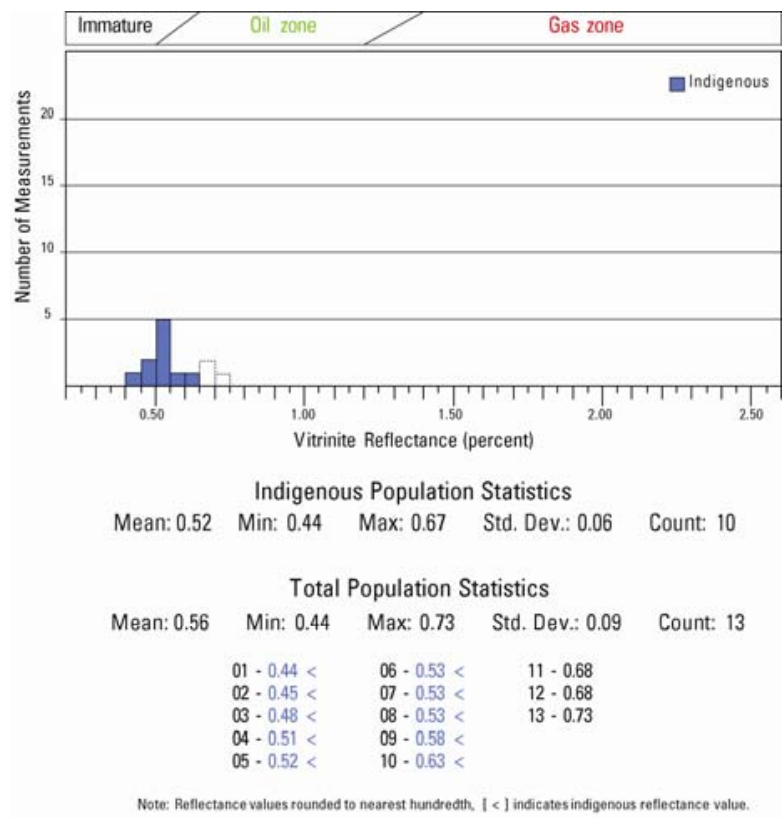

Figure 6. Histogram of $\mathrm{R}_{0}$ measurements on drill cuttings from $1,350-1,360 \mathrm{ft}$ in the Kerlyn Oil Company, Morrison No. 1 well, Young County, north-central Texas. $R_{0}=0.52$ based on 10 measurements. Measurements and histogram were provided by Humble Geochemical Services.

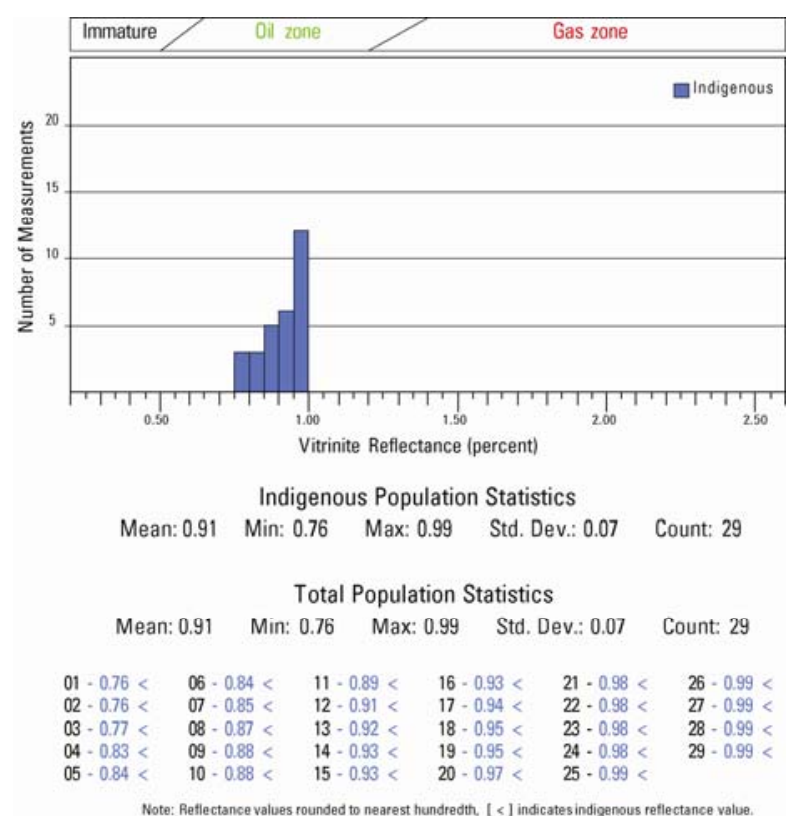

Figure 7. Histogram of $R_{0}$ measurements on drill cuttings from 2,030-2,040 ft in the Kerlyn Oil Company, Morrison 1 well, Young County, north-central Texas. $R_{0}=0.91$ based on 29 measurements. Measurements and histogram were provided by Humble Geochemical Services. 


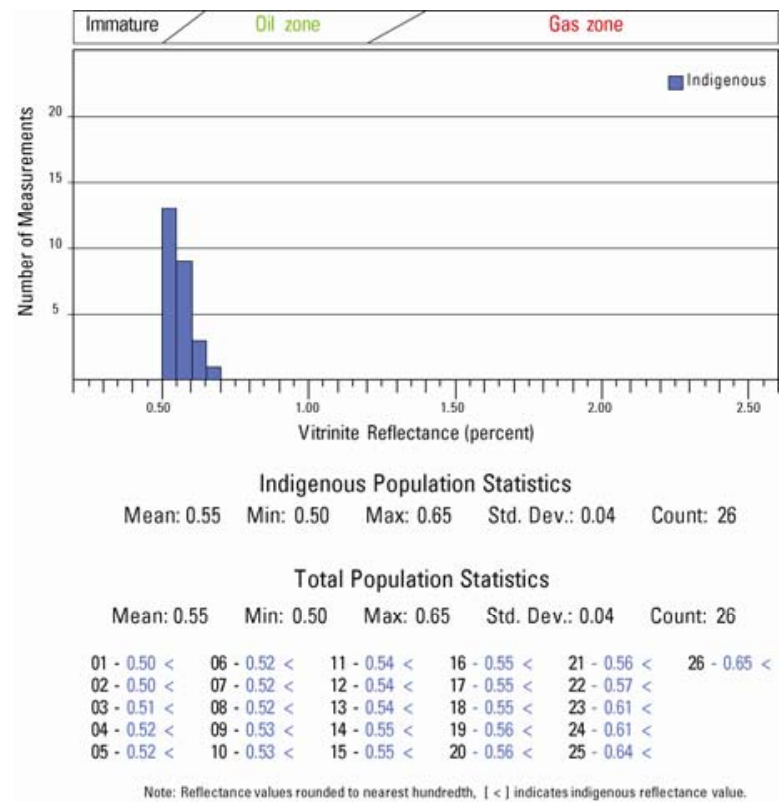

Figure 8. Histogram of $R_{0}$ measurements on drill cuttings from 2,160-2,170 ft in the Lipscomb and De Lange, Furr No. 1 well, Young County, north-central Texas. $\mathrm{R}_{0}=0.55$ based on 26 measurements. Measurements and histogram were provided by Humble Geochemical Services.

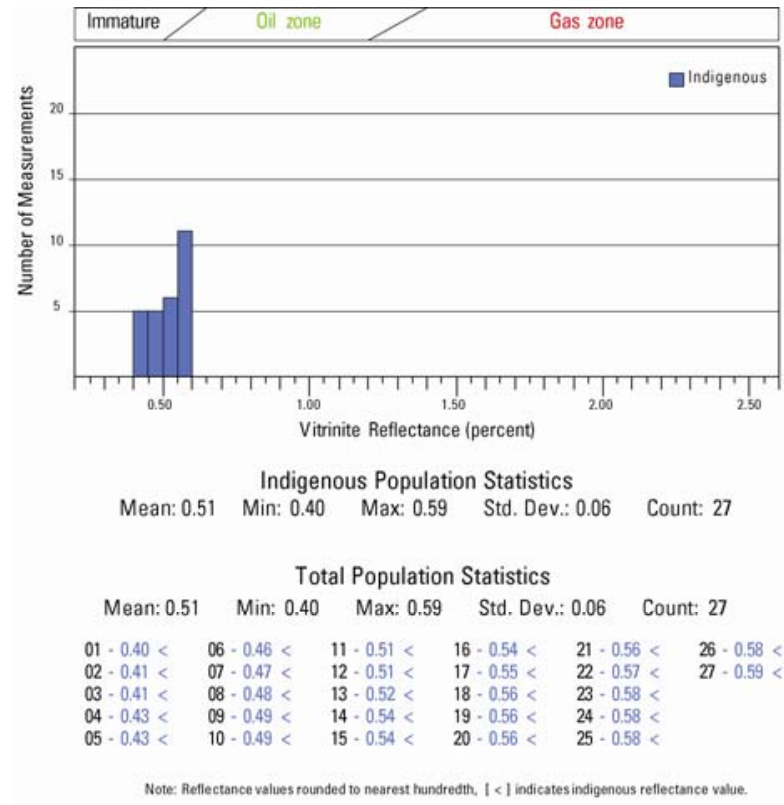

Figure 9. Histogram of $R_{0}$ measurements on drill cuttings from $780-790 \mathrm{ft}$ in the Panhandle Refining Co., Thompson No. 1 well, Young County, north-central Texas. $R_{0}=51$ based on 27 measurements. Measurements and histogram were provided by Humble Geochemical Services.

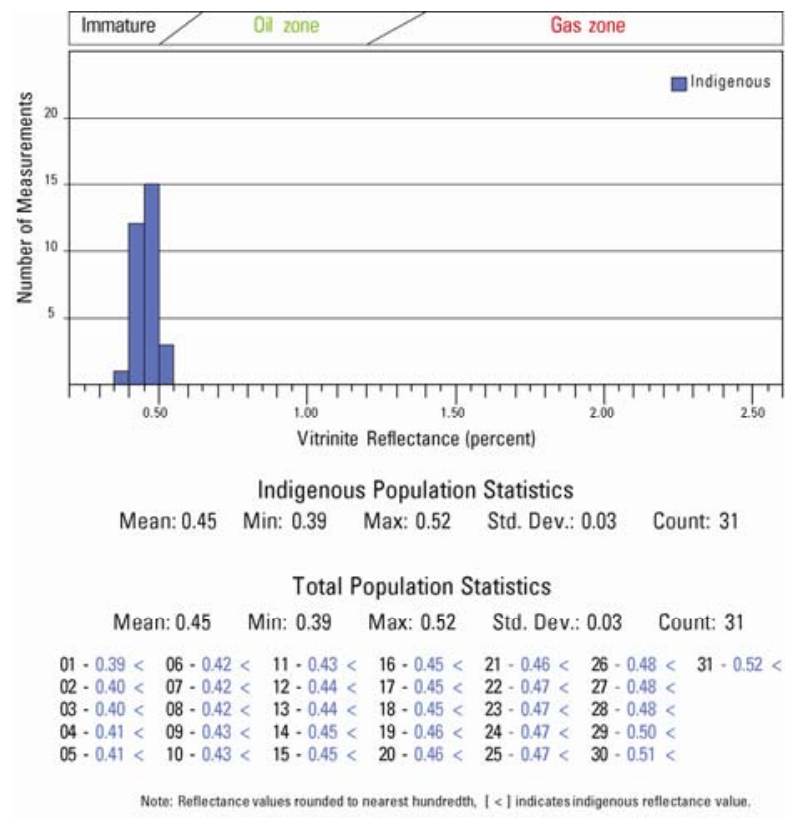

Figure 10. Histogram of $R_{0}$ measurements on drill cuttings from 1,630-1,640 ft in the Standard of Texas and H. Buchanan, Donnell No. 1 well, Young County, north-central Texas. $\mathrm{R}_{0}=0.45$ based on 31 measurements. Measurements and histogram were provided by Humble Geochemical Services.

\section{Coal rank in sample subset}

$\mathrm{R}_{0}$ measurements indicate that coal rank in the sample subset ranges from lignite $\left(\mathrm{R}_{0}=0.39\right.$ in middle Cisco Group, Morrison No. 1 well) to high volatile bituminous A coal $\left(\mathrm{R}_{0}=0.91\right.$ in basal Canyon Group, Morrison No. 1 well (Plate 1).

$R_{0}$ values also indicate subbituminous $B$ coal in the middle part of the Canyon Group in the Donnell No. 1 well $\left(\mathrm{R}_{0}=0.45\right)$, and subbituminous $\mathrm{A}$ coal in the upper part of the Strawn $\left(\mathrm{R}_{0}=0.63\right)$ and Canyon $\left(\mathrm{R}_{0}=0.61\right)$ groups in the Baldwin No. 1 well, the lower part of the Canyon Group in the Furr No. 1 well $\left(\mathrm{R}_{0}=0.55\right)$, and in the middle part of the Canyon Group in the Morrison No. $1\left(\mathrm{R}_{0}=0.52\right)$ and Thompson No. $1\left(\mathrm{R}_{0}=0.51\right)$ wells.

Coal rank in the sample subset should be reassessed using the results of follow-up $\mathrm{R}_{0}$ analysis.

\section{Summary and Proposed Future Work}

Collection and analysis of subsurface coal samples during the April 2005-February 2006 activities of the NCRDS State Cooperative program was extended to Young County, north-central Texas. This work builds upon a pilot study of coal rank assessment that began in adjacent Archer County. The study demonstrates the feasibility of using drill cuttings to recognize coal occurrences in the shallow subsurface of north-central 
Texas (at depths of up to about 2,000 ft), and to assess coal rank by means of $\mathrm{R}_{0}$ measurements on drill cuttings.

A total of 57 samples were collected from five wells. The collected samples represent coal and coaly shale beds in the Strawn (Pennsylvanian), Canyon (Pennsylvanian), and Cisco (Pennsylvanian-Permian) Groups. $R_{0}$ measurements on a sample subset indicate that coal rank ranges from lignite to high volatile subbituminous A coal.

Future work should include the further identification of subsurface coal and estimation of coal thickness using porosity logs (e.g., density and sonic). In addition, the study should be extended to eastern Stephens and northwestern Palo Pinto Counties (fig. 1). Further, field studies at representative coal outcrops in north-central Texas should be used to document vertical and lateral stratigraphic characteristics and trends which could then be used to assess coal occurrences and thickness estimates determined thorough the use of porosity logs.

\section{Acknowledgements}

Susan Tewalt and Peter Warwick of USGS provided support and guidance throughout the study. Special thanks to Dr. Robert W. Hook, Austin, Texas, who suggested extension of the pilot study to Young County, and made useful recommendations for coal sample collection. L. F. Brown, Jr., provided insight into the stratigraphy of north-central Texas, Pennsylvanian coalbearing intervals. Comments by Stephen C. Ruppel, Eric Potter and L. F. Brown, Jr., of the BEG and Sharon Swanson and Robert Milici of USGS improved the manuscript. Rosalba Mendoza of BEG digitized scanned images of the electric logs used and prepared computer displays of the well log cross section and individual digital logs. Daniel Ortuño of BEG facilitated access to the well logs and coordinated log scanning, and James Donnelly of BEG provided access to the drill cuttings. Editing was by Lana Dieterich of BEG, and graphics were by Joel L. Lardon of BEG's Media Information Technologies.

\section{References}

Brown, L. F., Jr., 1959, Problems of stratigraphic nomenclature and classification, Upper Pennsylvanian, North-Central Texas: American Association of Petroleum Geologists Bulletin, v. 43, no. 12, p. 2866-2871.

Brown, L. F., Jr., Goodson, J. L., and Harwood, Peggy, 1972, Abilene sheet, in Barnes, V. E., project director, Geologic atlas of Texas: Bureau of Economic Geology, scale 1:250,000.
Brown, L. F., Jr., 1973, Cisco depositional systems of North-Central Texas, in Brown, L. F., Jr., Cleaves, A. W., II, and Erxleben, A. W., Pennsylvanian depositional systems in North-Central Texas-A guide for interpreting terrigenous clastic facies in a cratonic basin: Bureau of Economic Geology Guidebook No. 14, p. 57-73.

Brown, L. F., Jr., Solís-Iriarte, R. F., and Johns, D. A., 1987, Regional stratigraphic cross sections, Upper Pennsylvanian and Lower Permian strata (Virgilian and Wolfcampian Series), North-Central Texas: Bureau of Economic Geology cross sections, 12 sheets.

Brown, L. F., Jr., Solís-Iriarte, R. F., and Johns, D. A., 1990, Regional depositional systems tracts, paleogeography, and sequence stratigraphy, Upper Pennsylvanian and Lower Permian strata, North- and West-Central Texas: Bureau of Economic Geology Report of Investigations No. 197, 116 p.

Cleaves, A. W., II, 1973, Depositional systems in the upper Strawn Group of North-Central Texas, in Brown, L. F., Jr., Cleaves, A. W., II, and Erxleben, A. W., Pennsylvanian depositional systems in North-Central Texas-A guide for interpreting terrigenous clastic facies in a cratonic basin: Bureau of Economic Geology Guidebook No. 14, p. 31-42.

Cleaves, A. W., II, 1975, Upper Desmoinesian-Lower Missourian depositional systems (Pennsylvanian), North-Central Texas: The University of Texas at Austin, Ph.D. dissertation, 257 p.

Erxleben, A. W., 1973, Depositional systems in the Pennsylvanian Canyon Group of North-Central Texas, in Brown, L. F., Jr., Cleaves, A. W., II, and Erxleben, A. W., Pennsylvanian depositional systems in North-Central Texas-A guide for interpreting terrigenous clastic facies in a cratonic basin: Bureau of Economic Geology Guidebook No. 14, p. 57-73.

Erxleben, A. W., 1974, Depositional systems in the Pennsylvanian Canyon Group of North-Central Texas: The University of Texas at Austin, M.A. thesis, $202 p$.

Evans, T. J., 1974, Bituminous coal in Texas: Bureau of Economic Geology Handbook 4, $65 \mathrm{p}$

Galloway, W. E., and Brown, L. F., Jr., 1972, Depositional systems and shelf-slope relationships in upper Pennsylvanian rocks, NorthCentral Texas: The University of Texas at Austin, Bureau of Economic Geology Report of Investigations No. 75, 62 p.

Guevara, E. H., and Sakurai, S., 2005, Subsurface Pennsylvanian coal and coaly shale samples, Archer County, North-Central Texas: Pilot cuttings sampling for coal-rank determination: FY04 Annual Technical Report submitted to NCRDS State Cooperative Program, U. S. Geological Survey, Award Number 02ERAG0006, 11 p, 9 figs, 4 tables, 2 appendices.

Kim, E. M., 2003, Pennsylvanian bituminous coal, North-Central Texas: potential for coalbed methane resource development: The University of Texas at Austin, Bureau of Economic Geology, FY03 Annual Technical Final Report for NCRDS State Cooperative Program prepared for the U.S. Geological Survey, $17 \mathrm{p}$.

Mapel, 1967, Bituminous coal resources of Texas: U.S. Geological Survey Bulletin 1242-D, 28 p.

Wermund, E. G., and Jenkins, W. A., Jr., 1970, Recognition of deltas by fitting trend surfaces to Upper Pennsylvanian sandstones in North-Central Texas, in Morgan, J. P., ed., Deltaic sedimentation, modern and ancient: Society of Economic Paleontologists and Mineralogists Special Publication 15, p. 256-269. 


\section{Appendix. Coal and coaly shale in drill cuttings descriptions, Young County, north-central Texas}




\begin{tabular}{|c|c|c|c|c|c|c|}
\hline $\begin{array}{l}\text { Occurrence } \\
\text { No. }\end{array}$ & $\begin{array}{l}\text { Well name / } \\
\text { location }\end{array}$ & $\begin{array}{c}\text { Elevation } \\
\quad(\mathrm{ft})\end{array}$ & $\begin{array}{c}\text { Total } \\
\text { depth (ft) }\end{array}$ & $\begin{array}{l}\text { Spud } \\
\text { date }\end{array}$ & $\begin{array}{l}\text { Compl; PB } \\
\text { dates }\end{array}$ & $\begin{array}{l}\text { Coal and black or dark-gray shale } \\
\text { occurrences (depth in ft) }\end{array}$ \\
\hline 1 & $\begin{array}{l}\text { Ajax Oil \& Development Co., C.R. Martin \#1 (dry WC) } \\
\text { G.C.Cottle Abst 65, } 112 \text { ac, } 864 \text { ft FNEL \& } 330 \text { ft FSEL } \\
\text { of Survey, } 4 \text { mi NE fr Ellesville }\end{array}$ & & 2451 & $7 / 21 / 1957$ & $\begin{array}{l}\text { 7/28/1957; } \\
8 / 20 / 1957\end{array}$ & $\begin{array}{l}\text { Sh, blk: } 310 \\
\text { coal: } 1260\end{array}$ \\
\hline 2 & $\begin{array}{l}\text { Akin \& Dimock, Harris Wilson \#1 (dry WC) } \\
\text { TE\&LL \#67 Sur Abst } 343,200 \mathrm{ac}, 900 \mathrm{ft} \text { Eof WL, } 1750 \mathrm{ft} \\
\text { N of SL ( } 330 \mathrm{ft} \text { FSL, } 990 \mathrm{ft} \text { FWL), } 6 \text { mi SW fr Olney }\end{array}$ & & 5112 & 10/1/1953 & $\begin{array}{l}10 / 19 / 1953 ; \\
10 / 120 / 1953\end{array}$ & coal: 2456 \\
\hline 3 & $\begin{array}{l}\text { Akin \& Rayzer et al., O. Deitrich \#1 (oil prod) } \\
\text { Geo. W. Stell Suv., Blk A-256, } 150 \text { ft from N \& E lines }\end{array}$ & $1204 \mathrm{GL}$ & 1102 & 8/21/1930 & 9/1/1930 & coal: 1102 \\
\hline 4 & $\begin{array}{l}\text { Akin \& Rayzer, J. Winkler \#1 (oil prod) } \\
\text { J. Poitevent Suv. Abst. 226, } 150 \mathrm{ft} f \mathrm{r} N \& \text { W lines }\end{array}$ & 1206 & 1089 & $5 / 226 / 1930$ & 6/13/1930 & Sh, blk: 1058 \\
\hline 5 & $\begin{array}{l}\text { Akin \& Rayzer et al., J.A. Winkler \#3 (oil prod) } \\
\text { J. Poitevent Suv., Blk A-226, Sec } 1.150 \mathrm{ft} f \mathrm{fr} \text { \& } \\
450 \mathrm{ft} \text { W lines }\end{array}$ & & 1094 & 10/2/1930 & 11/4/1930 & $\begin{array}{l}\text { Sh, blk: } 655 \\
\text { Sh, blk: } 1070\end{array}$ \\
\hline 6 & $\begin{array}{l}\text { C.W. Alcorn et al., 0.S. Perkins \#1 (oil prod) } \\
\text { TE\&L Suv, Blk 206, } 60 \text { ac Tr., } 150 \text { ft fr } S \& \text { E lines }\end{array}$ & 1207 & 740 & 12/26/1934 & $1 / 2 / 1935$ & $\begin{array}{l}\text { Sh, blk: } 200 \\
\text { Sh, blk: } 220 \\
\text { Sh, blk: } 370 \\
\text { Sh, blk: } 440 \\
\text { Sh, blk: } 729\end{array}$ \\
\hline 7 & $\begin{array}{l}\text { C.W. Alcorn, 0.S. Perkins \#2 (dry WC) } \\
\text { TE\&L Co. Suv, Blk 206, } 150 \mathrm{ft} f \mathrm{f} \text { S \& W lines, } \\
\mathrm{N} 1 / 2 \text { of Suv. }\end{array}$ & 1202 & 1/14/1902 & $2 / 26 / 1935$ & $3 / 6 / 1935$ & $\begin{array}{l}\text { Sh, blk: } 200 \\
\text { Sh, blk: } 440 \\
\text { Sh, blk: } 734\end{array}$ \\
\hline 8 & $\begin{array}{l}\text { Geo. A. Alexander Jr., Jennie Jeffery \#1 (dry hole) } \\
\text { TE\&L Co. Suv., } 150 \text { ft each way fr NW Cor., } \\
\text { N 1/2 of Blk } 45\end{array}$ & & 903 & 2/26/1931 & 3/14/1931 & Sh, blk: 777 \\
\hline 9 & $\begin{array}{l}\text { Geo. A. Alexander Jr., Jennie K. Jeffery \#2A (dry hole) } \\
\text { TE\&L Co. Suv., BIK 41, } 150 \mathrm{ft} f \mathrm{SL} \& \mathrm{WL}\end{array}$ & & 818 & 11/5/1930 & 11/12/1930 & $\begin{array}{c}\text { coal: } 298 \\
\text { Sh, blk: } 124 \\
\text { Sh, blk: } 340 \\
\text { Sh, blk: } 375 \\
\text { Sh, blk: } 708\end{array}$ \\
\hline 10 & $\begin{array}{l}\text { Geo. A. Alexander Jr., Jeffery Estate \#1 (dry hole) } \\
\text { TE\&L Co. Suv., Blk 56, Abst 332, } 150 \mathrm{ft} \text { fr NL \& EL, } \\
\text { SW 1/4 Suv 56, }\end{array}$ & 1199 & 1025 & 9/10/1930 & 9/20/1930 & $\begin{array}{l}\text { Sh, blk: } 577 \\
\text { Sh, blk: } 910\end{array}$ \\
\hline 11 & $\begin{array}{l}\text { Geo. A. Alexander Jr., Ward \#1 (dry hole) } \\
\text { TE\&L Co. Suv., Blk } 279,150 \mathrm{ft} \text { fr } S \text { \& E lines }\end{array}$ & 1179 & 701 & $1 / 10 / 1931$ & 1/16/1931 & $\begin{array}{l}\text { Sh, blk: } 140 \\
\text { Sh, blk: } 688\end{array}$ \\
\hline 12 & $\begin{array}{l}\text { H. B. Alexander et al., H.B. Alexander \#1 (dry hole) } \\
\text { BS\&F Suv., Blk Z, Abst. } 1670,150 \mathrm{ft} f \mathrm{fr} E \mathrm{EL} 150 \mathrm{ft} f \mathrm{fr} S \mathrm{SL}\end{array}$ & & 660 & $8 / 15 / 1930$ & $8 / 27 / 1930$ & $\begin{array}{l}\text { coal: } 123 \\
\text { Sh, blk: } 615\end{array}$ \\
\hline 13 & $\begin{array}{l}\text { H. B. Alexander, Jim Foster \#1 (dry hole) } \\
150 \mathrm{ft} \mathrm{NL} \& 150 \mathrm{ft} E \mathrm{~L}\end{array}$ & & & & & $\begin{array}{l}\text { coal: }: 184 \\
\text { coal: } 262\end{array}$ \\
\hline 14 & $\begin{array}{l}\text { H. B. Alexander, T.L. Oliver \#1 (dry hole) } \\
\text { TE\&L Suv., Blk 1940, approx. in center of } 130 \text { ac. tract }\end{array}$ & & 581 & $6 / 5 / 1932$ & $7 / 5 / 1932$ & $\begin{array}{l}\text { lignite: } 12 \\
\text { Sh, blk: } 520\end{array}$ \\
\hline 15 & $\begin{array}{l}\text { J.H. Alexander, H.F. Haygood \#1 (dry hole) } \\
\text { TE\&L Suv., } 102 \text { ac, } 150 \mathrm{ft} \text { fr NL \& } 550 \mathrm{ft} \mathrm{fr} \text { line }\end{array}$ & & 750 & $3 / 18 / 1936$ & $4 / 7 / 1936$ & $\begin{array}{l}\text { coal: } 390 \\
\text { Sh, blk: } 721\end{array}$ \\
\hline 16 & $\begin{array}{l}\text { J.R. Alexander, Belknap Coal Co. Corp. \#1 (oil prod) } \\
\text { TE\&L Suv. \#23, } 150 \mathrm{ft} \text { fr } \mathrm{N} \& \text { W lines }\end{array}$ & & 660 & 3/14/1936 & $5 / 12 / 1936$ & $\begin{array}{l}\text { Sh, blk: } 259 \\
\text { Sh, blk: } 295 \\
\text { Sh, blk: } 348\end{array}$ \\
\hline 17 & $\begin{array}{l}\text { J.R. Alexander, M.M. Burrus \#1 (dry hole) } \\
\text { TE\&\&L Suv., Sec. } 1365 \text {, approx. } 150 \mathrm{ft} \text { fr } S \text { \& E lines }\end{array}$ & & 802 & 8/23/1933 & 9/3/1933 & Sh, blk: 450 \\
\hline 18 & $\begin{array}{l}\text { J.R. Alexander \& W.E. Nunneley, Prideaux Brothers \#3 } \\
\text { (dry hole), YCS Lds. Suv., Blk } 15,150 \mathrm{ft} f \mathrm{fr} \& 450 \mathrm{ft} \\
\text { fr W lines } \\
\text { R. H. Alexander, Van Hamert \#1 (dry hole) }\end{array}$ & & 551 & $5 / 27 / 1937$ & $3 / 24 / 1937$ & $\begin{array}{l}\text { coal: } 71 \\
\text { Sh, blk: } 502\end{array}$ \\
\hline & $\begin{array}{l}\text { TE\&L Co. Ab. } 599,72 \text { ac., } 1050 \mathrm{ft} f \mathrm{fr} \& \& 900 \mathrm{ft} \text { fr } S \text { lines, } \\
41 / 2 \mathrm{mi} \mathrm{NW} \text { of Newcastle }\end{array}$ & & & & & Sh, blk: 671 \\
\hline 20 & $\begin{array}{l}\text { Samuel S. Alexander, R.O. Andrews \#1 (dry hole) } \\
\text { TE\&LL Co. Suv., Blok 1806, } 150 \text { ft each way fr NE Cor., } \\
\text { NW } 1 / 4 \text { of Blk }\end{array}$ & 1100 & 955 & $7 / 3 / 1936$ & 7/10/1936 & $\begin{array}{l}\text { Sh, blk: } 430 \\
\text { Sh, blk: } 826\end{array}$ \\
\hline 21 & $\begin{array}{l}\text { Samuel S. Alexander, W.L. Boyd \#1 (abd, dry hole) } \\
\text { TE\&L Co. Suv, Sec. } 1455,150 \mathrm{ft} \text { fr E \& } 950 \mathrm{ft} \text { fr N lines }\end{array}$ & & 871 & 7/30/1937 & $8 / 6 / 1937$ & $\begin{array}{l}\text { coal: } 416 \\
\text { Sh, blk: } 354\end{array}$ \\
\hline 22 & $\begin{array}{l}\text { Samuel S. Alexander, W.L. Boyd \#2 (abd, dry hole) } \\
\text { TE\&L Co. Suv, Blk } 1455,80 \text { Ac., } 1147 \mathrm{ft} \text { fr E \& } 150 \mathrm{ft} f r \\
\mathrm{~N} \text { lines, SE } 1 / 4 \text { of Blk } 1455 .\end{array}$ & & 1110 & $8 / 11 / 1937$ & $8 / 19 / 1937$ & $\begin{array}{l}\text { coal: } 410 \\
\text { Sh, bl:k } 328 \\
\text { Sh, blk: } 346 \\
\text { Sh, blk: } 812 \\
\text { Sh, blk: } 940\end{array}$ \\
\hline 23 & $\begin{array}{l}\text { Samuel S. Alexander, Clark \#1 (oil prod) } \\
\text { TE\&L Co. Suv, Sec. 209, } 160 \text { Ac. Tract, } 1050 \text { ft fr NL \& } \\
1950 \text { ft fr WL }\end{array}$ & 1162 & 850 & 3/25/1937 & 4/5/1937 & $\begin{array}{l}\text { Sh, blk: } 450 \\
\text { Sh, dark: } 485 \\
\text { Sh, blk: } 818\end{array}$ \\
\hline 24 & $\begin{array}{l}\text { Samuel S. Alexander, Mrs. L.D. Clark \#2 (oil prod) } \\
\text { TE\&L Co. Suv, Sec. } 209,1050 \mathrm{ft} \text { Sf NL \& } 1587 \mathrm{ft} f \mathrm{fr} \text { line } \\
300 \mathrm{ft} \text { E \& } 115 \mathrm{ft} \text { Sof \#1 well }\end{array}$ & & 848 & 4/9/1937 & 4/22/1937 & $\begin{array}{l}\text { Sh, blk: } 806 \\
\text { Sh, blk: } 841\end{array}$ \\
\hline 25 & $\begin{array}{l}\text { Samuel S. Alexander, L.D. Clark \#3 (oil prod) } \\
\text { TE\&L Co. Suv, Sec. } 209,160 \text { Ac., } 150 \mathrm{ft} \text { fr NL \& } \\
1050 \mathrm{ft} \text { fr E line }\end{array}$ & & 841 & $6 / 10 / 1937$ & $6 / 18 / 1937$ & $\begin{array}{l}\text { Sh, blk: } 215 \\
\text { Sh, blk: } 415 \\
\text { Sh, blk: } 475 \\
\text { Sh, blk: } 804\end{array}$ \\
\hline 26 & $\begin{array}{l}\text { S. S. Alexander, Clark \#4 (dry hole) } \\
\text { TE\&L Co. Suv, Sec. } 209,150 \mathrm{ft} \text { fr NL \& } 300 \mathrm{ft} W \text { of \#3 }\end{array}$ & 1157 & 1112 & 6/19/1937 & 6/28/1937 & $\begin{array}{l}\text { Sh, blk: } 410 \\
\text { Sh, blk: } 468 \\
\text { Sh, blk: } 812 \\
\text { Sh, blk: } 987\end{array}$ \\
\hline 27 & $\begin{array}{l}\text { Samuel S. Alexander, Mrs. L.D. Clark \#5 (oil prod) } \\
\text { TE\&L Co. Suv, Sec. 209, } 160 \text { Ac., } 150 \mathrm{ft} f \mathrm{NL} \text { \& } \\
750 \mathrm{ft} f \mathrm{EL} \text {. }\end{array}$ & 1153 & 839 & 6/23/1937 & 6/30/1938 & $\begin{array}{l}\text { Sh, blk: } 412 \\
\text { Sh, blk: } 477 \\
\text { Sh, blk: } 796\end{array}$ \\
\hline 28 & $\begin{array}{l}\text { Samuel S. Alexander, Mrs. L.D. Clark \#6 (dry hole) } \\
\text { TE\&L Co. Suv, Sec. 209, } 160 \text { Ac Tract, } 300 \mathrm{ft} \text { fr NL \& } \\
1050 \mathrm{ft} f \mathrm{fr} \text { EL, Olney field }\end{array}$ & 1155.4 & 875 & $7 / 2 / 1937$ & $7 / 12 / 1937$ & $\begin{array}{l}\text { Sh, blk: } 464 \\
\text { Sh, blk: } 825\end{array}$ \\
\hline 29 & $\begin{array}{l}\text { Samuel S. Alexander, Mrs. L.D. Clark \#7 (abd, dry hole) } \\
\text { TE\&L Co. Suv, Sec. 209, } 300 \mathrm{ft} \text { NW of \#1 }\end{array}$ & & 858 & 7/14/1937 & 7/19/1937 & $\begin{array}{l}\text { Sh, blk: } 235 \\
\text { Sh, bl: } 330 \\
\text { Sh, blk: } 492 \\
\text { Sh, blk: } 615 \\
\text { Sh, blk: } 817\end{array}$ \\
\hline 30 & $\begin{array}{l}\text { S. S. Alexander, Irene Gathright \#1 (dry hole) } \\
\text { TE\&L Co. Suv, Blk } 1801,150 \mathrm{ft} f \mathrm{fr} \text { WL \& } 783 \mathrm{ft} \text { SL of } \\
\text { SE } 1 / 2 \text { of BIK } 1801\end{array}$ & 1114 & 925 & $7 / 14 / 1936$ & 7/19/1936 & coal: 455 \\
\hline 31 & $\begin{array}{l}\text { S. S. Alexander, W.B. Howard \#3 (oil prod) } \\
\text { TE\&L Co. Suv, Blk } 3401,15 \text { ac., } 150 \mathrm{ft} \text { f the N \& W lines }\end{array}$ & & 904 & $2 / 10 / 1936$ & $2 / 16 / 1936$ & $\begin{array}{l}\text { Sh, blk: } 250 \\
\text { Sh, bl:k: } 445 \\
\text { Sh, blk: } 515 \\
\text { Sh, blk: } 858\end{array}$ \\
\hline 32 & $\begin{array}{l}\text { S. S. Alexander et al., W.B. Howard \#2 (oil prod) } \\
\text { TE\&L Co. Suv, Fr. "C", Sec. } 3401,15 \text { Ac. Lease, } 210 \mathrm{ft} \\
\text { fr SL \& } 210 \mathrm{ft} f \mathrm{fr} \text { WL }\end{array}$ & 1196 & 928 & 11/29/1935 & $12 / 14 / 1935$ & $\begin{array}{l}\text { Sh, blk: } 475 \\
\text { Sh, blk: } 530 \\
\text { Sh, blk: } 540 \\
\text { Sh, blk: } 889\end{array}$ \\
\hline 33 & $\begin{array}{l}\text { S. S. Alexander et al., W.B. Howard \#5 (oil prod) } \\
\text { TE\&L Co. Suv, Blk } 3401,15 \text { Ac., } 150 \mathrm{ft} \text { fr NL \& } 210 \mathrm{ft} \text { fr EL }\end{array}$ & & 912 & 3/30/1936 & 4/9/1936 & $\begin{array}{l}\text { Sh, blk: } 267 \\
\text { Sh, blk: } 442 \\
\text { Sh, blk: } 868\end{array}$ \\
\hline 34 & $\begin{array}{l}\text { Samuel S. Alexander Oil Co., W.R. Goldston \#1B (dry hole) } \\
\text { TE\&LL Co. Suv, Sec. 2057, Blk 9, } 330 \mathrm{ft} \text { fr N \& W lines }\end{array}$ & 1229 & 4550 & 9/4/1936 & 1/25/1937 & $\begin{array}{l}\text { Sh, blik: } 888 \\
\text { Sh, blk: } 1280 \\
\text { Sh, blk: } 1450\end{array}$ \\
\hline
\end{tabular}




\begin{tabular}{|c|c|c|c|c|c|c|}
\hline $\begin{array}{l}\text { Occurrence } \\
\text { No. }\end{array}$ & $\begin{array}{l}\text { Well name / } \\
\text { location }\end{array}$ & $\begin{array}{c}\text { Elevation } \\
\text { (ft) }\end{array}$ & $\begin{array}{c}\begin{array}{c}\text { Total } \\
\text { depth (ft) }\end{array}\end{array}$ & $\begin{array}{l}\text { Spud } \\
\text { date }\end{array}$ & $\begin{array}{l}\text { Compl; PB } \\
\text { dates }\end{array}$ & $\begin{array}{l}\text { Coal and black or dark-gray shale } \\
\text { occurrences (depth in ft) }\end{array}$ \\
\hline 35 & $\begin{array}{l}\text { Samuel S. Alexander Oil Corp., G.B. Hamilton Estate \#5 } \\
\text { (dry hole) TE\&L Co. Suv, Blk } 3401,40 \text { ac., } 750 \mathrm{ft} f \mathrm{fr} \text { EL \& } \\
250 \mathrm{ft} \text { fr NL. }\end{array}$ & & 902 & 6/13/1936 & 6/18/1936 & Sh, blk: 859 \\
\hline 36 & $\begin{array}{l}\text { Samuel S. Alexander Oil Corp., G.B. Hamilton Estate \#6 } \\
\text { (oil prod) TE\&L Co. Suv, Blk } 3401,40 \text { ac., } 450 \mathrm{ft} \mathrm{fr} \mathrm{EL} \mathrm{\&} \\
470 \mathrm{ft} f \mathrm{fr} \text { SL }\end{array}$ & 1182 & 914 & $1 / 13 / 1937$ & 1/19/1937 & $\begin{array}{l}\text { Sh, blk: } 435 \\
\text { Sh, blk: } 440 \\
\text { Sh, blk: } 870\end{array}$ \\
\hline 37 & $\begin{array}{l}\text { Samuel S. Alexander Oil Corp., G.B. Hamilton Estate, } \# 7 \\
\text { (dry hole) TE\&L Co. Suv, Blk } 3401,40 \text { Ac. Lse., } 440 \mathrm{ft} \mathrm{N} \& \\
750 \mathrm{ft} \text { W of SE corner of } 40 \text { Ac. Lse. }\end{array}$ & & 950 & $1 / 30 / 1937$ & $2 / 9 / 1937$ & $\begin{array}{l}\text { Sh, blk: } 445 \\
\text { Sh, blk: } 522 \\
\text { Sh, blk: } 880\end{array}$ \\
\hline 38 & $\begin{array}{l}\text { Samuel S. Alexander Oil Corp., Hamilton Estate \#8 } \\
\text { TE\&LL Suv. } 3401 \text {, Abs. } 1221,40 \text { ac., } 450 \mathrm{ft} f \mathrm{fr} \text { EL, } 150 \mathrm{ft} F \mathrm{SL}\end{array}$ & & & & & Sh, blk: 871 \\
\hline 39 & $\begin{array}{l}\text { Samuel S. Alexander Oil Corp., G.B. Hamilton \#9 } \\
\text { TE\&LL Suv. Blk } 3401,40 \text { Acre Tract, } 450 \mathrm{ft} \text { south \& } 450 \mathrm{ft} \\
\text { west fr NE corner Blk } 3401\end{array}$ & 1172 & $7 / 1 / 1902$ & $5 / 12 / 1937$ & $5 / 21 / 1937$ & $\begin{array}{l}\text { Sh, blk: } 440 \\
\text { Sh, blk: } 505 \\
\text { Sh, blk: } 857\end{array}$ \\
\hline 40 & $\begin{array}{l}\text { S. S. Alexander Oil Corp., G.B. Hamilton \#10 (dry hole) } \\
\text { TE\&L Co. Suv., Blk } 3401,40 \text { Acres, } 750 \mathrm{ft} f \mathrm{E} \text { \& } \\
450 \mathrm{ft} f \mathrm{~N} \text { lines }\end{array}$ & & 1005 & 5/29/1937 & $6 / 6 / 1937$ & $\begin{array}{l}\text { Sh, blk: } 245 \\
\text { Sh, blk: } 436 \\
\text { Sh, blk: } 510 \\
\text { Sh, blk: } 873\end{array}$ \\
\hline 41 & $\begin{array}{l}\text { Samuel S. Alexander Oil Corp., G.B. Hamilton Estate \#6A } \\
\text { (dry hole) TE\&L Suv., Blk } 3401,185 \mathrm{ftff} \text { f EL \& } 700 \mathrm{ft} \text { fr NL }\end{array}$ & & 950 & $1 / 3 / 1938$ & 1/15/1938 & $\begin{array}{l}\text { Sh, blk: } 465 \\
\text { Sh, blk: } 538 \\
\text { Sh, blk: } 871\end{array}$ \\
\hline 42 & $\begin{array}{l}\text { Alexander \& Beavers, G.B. Hamilton Estate \#1 (oil prod) } \\
\text { TE\&LL Suv., BIK 3401, well located in NE corner }\end{array}$ & & 905 & 1/27/1936 & 2/5/1936 & $\begin{array}{l}\text { Sh, blk: } 238 \\
\text { Sh, blk: } 341 \\
\text { Sh, blk: } 460 \\
\text { Sh, blk: } \\
\text { Sh, blk: } 615 \\
\end{array}$ \\
\hline 43 & $\begin{array}{l}\text { Alexander \& Beavers, G.B. Hamilton \#2 (oil prod) } \\
\text { TE\&LL Suv., Blk } 3401,40 \text { ac. Tr., } 150 \mathrm{ft} \text { FEL \& } 750 \mathrm{ft} \text { FNL }\end{array}$ & & 919 & 2/26/1936 & & $\begin{array}{l}\text { Sh, blk: } 245 \\
\text { Sh, blk: } 448 \\
\text { Sh, blk: } 287 \\
\text { Sh, blk: } 877\end{array}$ \\
\hline 44 & $\begin{array}{l}\text { Alexander \& Beavers, G.B. Hamilton Estate \#3 (oil prod) } \\
\text { TE\&L Suv., Blk 3401, } 40 \text { ac. Tr., } 450 \mathrm{ft} \text { FEL \& } 150 \mathrm{ft} \text { FNL }\end{array}$ & & 902 & $3 / 5 / 1936$ & 3/17/1936 & $\begin{array}{l}\text { Sh, blk: } 305 \\
\text { Sh, blk: } 460 \\
\text { Sh, blk: } 857\end{array}$ \\
\hline 45 & $\begin{array}{l}\text { Alexander \& Beavers, G.B. Hamilton Estate \#4 (dry hole) } \\
\text { TE\&L Suv., Blk } 3401,150 \mathrm{ft} \text { FEL \& } 450 \mathrm{ft} \text { FNL }\end{array}$ & & 1000 & 3/15/1936 & 3/24/1936 & $\begin{array}{l}\text { Sh, blk: } 480 \\
\text { Sh, blk: } 519 \\
\text { Sh, blk: } 869\end{array}$ \\
\hline 46 & 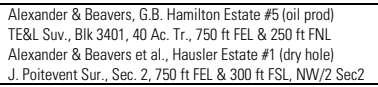 & & $\begin{array}{l}902 \\
1102\end{array}$ & $\begin{array}{l}6 / 13 / 1936 \\
4 / 20 / 1936\end{array}$ & $\begin{array}{l}6 / 18 / 1936 \\
4 / 30 / 1936\end{array}$ & Sh, blk: 1053 \\
\hline 47 & $\begin{array}{l}\text { Alexander-Clarida et al, E. Johnson \#2 (dry hole) } \\
\text { TE\&L Sur., Sec. A-921, Blk } 1495,100 \text { ac., } 450 \mathrm{ft} \mathrm{FNL} \mathrm{\&} \\
900 \mathrm{ft} F E L, 5 \text { mi NE of Jean }\end{array}$ & & 945 & $11 / 6 / 1955$ & $11 / 7 / 1955$ & $\begin{array}{c}\text { coal: } 385 \\
\text { coal: } 405 \\
\text { coal: } 428 \\
\text { coal: } 680 \\
\text { Sh, dk gy: } 853 \\
\text { Sh, blk: } 879\end{array}$ \\
\hline 48 & $\begin{array}{l}\text { Alexander-Clarida et al, E. Johnson \#4 (oil prod) } \\
\text { TE\&L Co. Suv., Sec. 1495, } 100 \text { ac., } 450 \mathrm{ft} \text { FNL \& } \\
1000 \mathrm{ft} \mathrm{FWL,} 5 \mathrm{mi} \text { NE of Jean }\end{array}$ & & 871 & 9/6/1955 & 9/7/1955 & $\begin{array}{c}\text { coal streaks: } 340 \\
\text { coal: } 360 \\
\text { coal: } 372 \\
\text { coal: } 384 \\
\text { Sh, blk: } 458 \\
\text { Sh, dk gy: } 478 \\
\text { coal: } 480 \\
\text { Sh, dk gy: } 825 \\
\text { Sh, blk: } 846\end{array}$ \\
\hline 49 & $\begin{array}{l}\text { Alexander-Clarida et al, E. Johnson \#6 (abd, dry hole) } \\
\text { TE\&L Co. Suv., Blk } 1495, \text { Sec. A-921, } 100 \text { ac., } 750 \mathrm{ft} \\
\text { FN\&EL of } S / 2,5 \text { mi NE Jean }\end{array}$ & & 930 & 9/13/1955 & $\begin{array}{l}\text { 9/15/1955; } \\
10 / 2 / 1955\end{array}$ & $\begin{array}{c}\text { coal: } 370 \\
\text { coal: } 391 \\
\text { coal: } 453 \\
\text { coal: } 466 \\
\text { Sh, blk: } 732 \\
\text { Sh, dk gy: } 838 \\
\text { Sh, blk: } 870\end{array}$ \\
\hline 50 & $\begin{array}{l}\text { W.E. Allaun, J.T. Sims \#1 (dry hole) } \\
\text { Suv. } 610,80 \text { Acres, } 150 \mathrm{ft} \text { FNL \& } 150 \mathrm{ft} \text { FWL of } \\
\text { SE } 1 / 4 \text { of Suv. }\end{array}$ & & 835 & $7 / 30 / 2028$ & $8 / 10 / 2028$ & $\begin{array}{c}\text { coal: } 91 \\
\text { coal, hard: } 100 \\
\text { Sh, blk: } 150 \\
\text { Sh, blk: } 237 \\
\text { Sh, blk: } 648 \\
\text { Sh, blk: } 760 \\
\text { Sh, sdy, blk: } 830\end{array}$ \\
\hline 51 & $\begin{array}{l}\text { F.J. Allbritain, F.M. Gilmore \#3 (oil prod) } \\
\text { Young Co. School Land, Blk 14, Abst. 1284, } 47 \text { Acres, } \\
450 \mathrm{ft} \text { NL \& } 750 \mathrm{ft} \mathrm{WL}\end{array}$ & & 584 & & $12 / 7 / 1933$ & Sh, blk: 546 \\
\hline 52 & $\begin{array}{l}\text { F.J. Allbritain, F.M. Gilmore \#5 (oil prod) } \\
\text { Young Co. School Lds, Blk 14, Abst. 1284, } 46 \text { Acres, } \\
450 \mathrm{ft} \text { FNL \& } 1350 \mathrm{ft} \text { FWL }\end{array}$ & & 585 & $10 / 1 / 1933$ & 10/10/1933 & Sh, blk: 549 \\
\hline 53 & $\begin{array}{l}\text { F.J. Allbritain, F.M. Gilmore \#8 (oil prod) } \\
\text { Young Co. School Lds, Blk } 14 \text {, Abst. 1284, } 150 \mathrm{ft} \text { FNL \& } \\
300 \mathrm{ft} \text { W of } \# 4 \text {, Abst. } 1284\end{array}$ & 1116 & 586 & $10 / 3 / 1936$ & 10/9/1936 & $\begin{array}{l}\text { Sh, blk: } 130 \\
\text { Sh, blk: } 551 \\
\text { Sh, blk: } 555\end{array}$ \\
\hline 54 & $\begin{array}{l}\text { F.J. Allbritain, F.M. Gilmore \#9 (oil prod) } \\
\text { YCSL Suv, Blk 14, Sec. 1284, well in NW corner Tr Abst } 1284\end{array}$ & & & & & Sh, blk: 555 \\
\hline 55 & $\begin{array}{l}\text { F.J. Allbritain, F.M. Gilmore \#10 (oil prod) } \\
\text { YCSL Suv, BIk 14, Sec. 1284, } 150 \mathrm{ft} \mathrm{FN} \mathrm{\&} \mathrm{W} \mathrm{lines}\end{array}$ & & 593 & 5/24/1937 & $5 / 31 / 1927$ & Sh, blk: 554 \\
\hline 56 & $\begin{array}{l}\text { F.J. Allbritain, F.M. Gilmore \#11 (oil prod-Markey field) } \\
\text { YCSL Suv, BIk 14, Sec. } 1284,47 \text { acres, } 390 \mathrm{ft} F N L \text { \& } \\
240 \mathrm{ft} \text { of } \# 9 \& 300 \mathrm{ft} W \text { of } \# 3\end{array}$ & & 586 & 4/18/1939 & 4/26/1939 & $\begin{array}{l}\text { coal: } 118 \\
\text { Sh, blk: } 558\end{array}$ \\
\hline 57 & $\begin{array}{l}\text { F.J. Allbritain, F.M. Gilmore \#14 (oil prod-Markey field) } \\
\text { YCSL Suv, Blk 14, Sec. 1284, } 47 \text { acres, } 450 \mathrm{ft} \text { FNL \& } \\
150 \mathrm{ft} \text { FWL }\end{array}$ & 1115 & 579 & $9 / 1 / 1945$ & 10/15/1945 & $\begin{array}{l}\text { coal: } 114 \\
\text { Sh, blk: } 119 \\
\text { Sh, blk: } 543\end{array}$ \\
\hline 58 & $\begin{array}{l}\text { F.J. Allbritain, Gregg \#4 (abd dry hole) } \\
\text { Young County School Suv. Blk } 17,150 \mathrm{ft} \text { fr } \& \& \text { W lines } \\
\text { of } 100 \text { acre Tr. }\end{array}$ & 1101 & 755 & 11/14/1935 & 11/26/1935 & $\begin{array}{l}\text { lignitie: } 135 \\
\text { Sh, blk: } 695\end{array}$ \\
\hline 59 & $\begin{array}{l}\text { F.J. Allbritain, Mrs. F..Gregg \#11 (dry hole) } \\
\text { YCSL Suv, Blk 17, Sec. } 1284,150 \mathrm{ft} \text { FWL \& } 450 \mathrm{ft} F S L\end{array}$ & 1115 & 579 & $9 / 1 / 1945$ & 10/15/1945 & $\begin{array}{l}\text { coal: } 132 \\
\text { Sh, blk: } 554\end{array}$ \\
\hline 60 & $\begin{array}{l}\text { F.J. Allbritain, W.W. Gregg f } 12 \text { (dry hole) } \\
\text { YCSL Suv, Blk 17, Sec. } 1284,100 \text { acres, } 450 \text { ft FNL \& } \\
500 \mathrm{ft} \mathrm{FWL}\end{array}$ & & 638 & $8 / 9 / 1937$ & 8/13/1937 & $\begin{array}{l}\text { Sh, blk: } 132 \\
\text { Sh, blk: } 548\end{array}$ \\
\hline 61 & $\begin{array}{l}\text { F.J. Allbritain, J.P. Logan \#1 (dry hole) } \\
\text { YCSLL Suv, Blk } 24,80 \text { acres, } 450 \mathrm{ft} F S L \text { \& } 750 \mathrm{ft} \text { FWL }\end{array}$ & & 611 & $8 / 2 / 1937$ & $8 / 6 / 1937$ & $\begin{array}{l}\text { Sh, blk: } 126 \\
\text { Sh, blk: } 560\end{array}$ \\
\hline 62 & $\begin{array}{l}\text { R.E. Allday, Jacob Cross et al. \#1 (dry hole) } \\
\text { TE\&LL Suv, Blk 1993, } 750 \mathrm{ft} \text { FNL \& } 150 \mathrm{ft} \mathrm{FEL}\end{array}$ & & 574 & 9/23/1929 & 9/29/1929 & $\begin{array}{c}\text { coal: } 95 \\
\text { coal: } 155 \\
\text { Sh, blk: } 567\end{array}$ \\
\hline 63 & $\begin{array}{l}\text { R.E. Allday, Est. C.W. Johnson \#1 (dry hole) } \\
\text { E.W. Sargent Suv. Bllk 6, Richardson Subd., } 150 \mathrm{ft} \text { FNL \& } \\
150 \mathrm{ft} \text { FEL }\end{array}$ & & 614 & 10/14/1929 & 10/20/1929 & $\begin{array}{l}\text { coal: } 160 \\
\text { Sh, blk: } 226 \\
\text { Sh, blk: } 557\end{array}$ \\
\hline 64 & $\begin{array}{l}\text { R.E. Allday, Petty \& Miller \#1 (dry hole) } \\
\text { TE\&L Suv, Blk } 3404,150 \text { ft FNL \& } 150 \text { ft FWL }\end{array}$ & & 593 & $11 / 18 / 1929$ & 12/12/1929 & $\begin{array}{l}\text { sil, thil. } 34 \\
\text { cont } 34 \\
\text { Sh, blk: } 238\end{array}$ \\
\hline
\end{tabular}




\begin{tabular}{|c|c|c|c|c|c|c|}
\hline $\begin{array}{l}\text { Occurrence } \\
\text { No. }\end{array}$ & $\begin{array}{l}\text { Well name / } \\
\text { location }\end{array}$ & $\begin{array}{l}\text { Elevation } \\
\text { (ft) }\end{array}$ & $\begin{array}{c}\text { Total } \\
\text { depth (ft) }\end{array}$ & $\begin{array}{l}\text { Spud } \\
\text { date }\end{array}$ & $\begin{array}{l}\text { Compl; PB } \\
\text { dates }\end{array}$ & $\begin{array}{l}\text { Coal and black or dark-gray shale } \\
\text { occurrences (depth in ft) }\end{array}$ \\
\hline 65 & $\begin{array}{l}\text { Allday Oil Corp., C.W. Johnson \#1 (dry hole) } \\
\text { E.W. Sargent Suv. Blk Lot \#1, Richardson Bros. Subd., } \\
150 \mathrm{ft} \text { FEL \& } 150 \mathrm{ft} F \mathrm{FL}\end{array}$ & 1204 & 604 & 2/15/1929 & 2/23/1929 & $\begin{array}{c}\text { coal: } 161 \\
\text { coal: } 228 \\
\text { Sh } 4 k 543\end{array}$ \\
\hline & $150 \mathrm{ft} \mathrm{FEL} \mathrm{\&} 150 \mathrm{ft} \mathrm{FSL}$ & & & & & Sh, blk: 543 \\
\hline 66 & $\begin{array}{l}\text { Allday Oil Corp., C.W. Johnson Est. \#6A (oil prod) } \\
\text { E.W. Sergeant Suv. Blk } 14,40 \text { ac., } 1080 \mathrm{ft} \text { FWL \& } 150 \mathrm{ft} \text { FNL }\end{array}$ & & 598 & 10/11/1928 & 10/18/1928 & $\begin{array}{l}\text { coal: } 175 \\
\text { coal: } 232\end{array}$ \\
\hline 67 & $\begin{array}{l}\text { Allday Oil Corp., C.W. Johnson Est. \#7A (dry hole) } \\
\text { E.W. Sergeant Suv. } 1390 \mathrm{ft} \text { FWL \& } 150 \mathrm{ft} \text { FNL }\end{array}$ & & 640 & 10/18/1928 & 10/24/1928 & $\begin{array}{l}\text { Sh, blk: } 180 \\
\text { Sh, blk: } 245 \\
\text { Sh, blk: } 568\end{array}$ \\
\hline 68 & $\begin{array}{l}\text { Allday Oil Corp., C.W. Johnson Est. . \#8A (oil prod) } \\
\text { E.W. Sergeant Sur. } 1080 \mathrm{ft} F \mathrm{FWL} \& 450 \mathrm{ft} F \mathrm{FL}\end{array}$ & & 594 & 10/19/1928 & 10/27/1928 & $\begin{array}{c}\text { coal: } 174 \\
\text { Sh, blk: } 560\end{array}$ \\
\hline 69 & $\begin{array}{l}\text { Allday Oil Corp., C.W. Johnson Est. } \# 5 B \text { (oil prod) } \\
\text { E.W. Sergeant Suv. Blk } 15,40 \text { ac., } 450 \mathrm{ft} \text { FWL \& } 194 \mathrm{ft} \text { FSL }\end{array}$ & & 509 & 10/24/1928 & 10/30/1928 & $\begin{array}{l}\text { Sh, blk: } 560 \\
\text { coal: } 175\end{array}$ \\
\hline 70 & $\begin{array}{l}\text { Allday Oil Corp., C.W. Johnson Est. \#6B (oil prod) } \\
\text { E.W. Sergeant Suv. Sec. } 15,450 \mathrm{ft} \text { \& } 194 \mathrm{ft} N \mathrm{fr} \text { SW corner }\end{array}$ & & & & & $\begin{array}{l}\text { Sh, blk: } 150 \\
\text { coal: } 220 \\
\text { Sh, blk: } 515\end{array}$ \\
\hline 71 & $\begin{array}{l}\text { Allday Oil Corp., C.W. Johnson Est. \#1C (oil prod) } \\
\text { E.W. Sergeant Sur. Blk } 8,150 \mathrm{ft} E \& 150 \mathrm{ft} \text { S of NW corner }\end{array}$ & & 509 & 10/1/1928 & 10/5/1928 & $\begin{array}{l}\text { Sh, blk: } 100 \\
\text { Sh, blk: } 175\end{array}$ \\
\hline 72 & $\begin{array}{l}\text { Allday Oil Corp., C.W. Johnson Est. \#2C (oil prod) } \\
\text { E.W. Sergeant SuR. Blk 8, } 150 \mathrm{ft} \text { \& } 150 \mathrm{ft} \text { S of NW corner }\end{array}$ & & 545 & 10/9/2028 & 10/14/2028 & $\begin{array}{l}\text { Sh, blk: } 108 \\
\text { Sh, blk: } 181 \\
\text { Sh, blk: } 473 \\
\text { Sh, blk: } 492 \\
\end{array}$ \\
\hline 73 & $\begin{array}{l}\text { Allday Oil Corp., J.P. McKinley. \#9 (dry hole) } \\
\text { TE\&L Suv, } 25 \text { Acres, Blk } 1489,450 \mathrm{ft} \text { FEL \& } 754 \mathrm{ft} \mathrm{FSL} \mathrm{of} \\
\text { S } 25 \text { Acres of E } 65 \text { Acres }\end{array}$ & & 535 & 9/26/1928 & 10/12/1928 & $\begin{array}{l}\text { coal: } 145 \\
\text { Sh, blk: } 512\end{array}$ \\
\hline 74 & $\begin{array}{l}\text { Allday Oil Corp., E.W. Sergent \#3A (oil prod) } \\
\text { E.W. Sergent Sur., } 450 \mathrm{ft} \text { S \& W fr NE Cor. S } 40 \text { Ac. of } \\
\text { W62 1/2 Ac. N } 125 \text { ac E } 450 \text { Ac. }\end{array}$ & & 592 & $4 / 16 / 1930$ & 4/24/1930 & $\begin{array}{l}\text { coal: } 167 \\
\text { Sh, blk: } 547\end{array}$ \\
\hline 75 & $\begin{array}{l}\text { Amerada Petroleum Corp., J.W. Daws \#1 (dry hole) } \\
\text { TE\&L Co. \#750 Surv., } 150 \mathrm{ft} \text { FEL \& } 150 \mathrm{ft} \text { FSL }\end{array}$ & & 965 & 10/10/1928 & $10 / 28 / 1928$ & $\begin{array}{l}\text { Sh, dark: } 230 \\
\text { Sh, blk: } 415 \\
\text { Sh, blk: } 435 \\
\end{array}$ \\
\hline 76 & $\begin{array}{l}\text { Amerada Petroleum Corp., J.H. Cochran \#1 (dry hole) } \\
\text { Pennington Sur, } 660 \mathrm{ft} \text { of } \mathrm{SL} \& 2200 \mathrm{ft} \text { of WL, } 2 \text { acre tract }\end{array}$ & & 536 & $3 / 26 / 1928$ & $4 / 5 / 1928$ & $\begin{array}{l}\text { Sh, dark: } 150 \\
\text { Sh, blk: } 442\end{array}$ \\
\hline 77 & $\begin{array}{l}\text { Amerada Petroleum Corp. L.M. Wilkinson \#1 (dry hole) } \\
\text { TE\&L Co. Survey Sec. } 724 \text { \& } 725,150 \mathrm{ft} \mathrm{FSL} \mathrm{\&} 1867 \mathrm{ft} \text { FEL }\end{array}$ & & 806 & $9 / 1 / 1928$ & 9/19/1928 & $\begin{array}{l}\text { Sh, blk: } 250 \\
\text { Sh, blk: } 335 \\
\text { Sh, dark: } 430 \\
\text { Sh, blk: } 520 \\
\text { Sh, dark: } 728\end{array}$ \\
\hline 78 & $\begin{array}{l}\text { O.T. Anderson, A.A. Bernhart } \# 1 \text { (dry hole) } \\
\text { W. McCarty Suv. Abst. } 1488,59 \text { acres, } 150 \mathrm{ft} F N \& \text { lines }\end{array}$ & & 1113 & & & Sh, blk: 660 \\
\hline 79 & $\begin{array}{l}\text { 0.T. Anderson Oil Co., Belknap Coal Co. \#1 (oil prod) } \\
\text { TE\&L Co. Suv. \#23, } 150 \mathrm{ft} \text { FWL \& } 900 \mathrm{ft} \text { FNL }\end{array}$ & & 654 & 2/17/1935 & $3 / 4 / 1935$ & $\begin{array}{l}\text { Sh, blk: } 163 \\
\text { Sh, blk: } 268 \\
\text { Sh, blk: } 317\end{array}$ \\
\hline 80 & $\begin{array}{l}\text { 0.T. Anderson 0il Co., Belknap Coal Co. \#3 (dry hole) } \\
\text { TE\&L Co. Suv. \#23, } 45.3 \text { Acre Tr., } 450 \mathrm{ft} \mathrm{FWL} \mathrm{\&} 150 \mathrm{ft} \text { FSL }\end{array}$ & & & & & $\begin{array}{l}\text { Sh, blk: } 267 \\
\text { Sh, blk: } 350 \\
\text { Sh, blk: } 441\end{array}$ \\
\hline 81 & $\begin{array}{l}\text { 0.T. Anderson Oil Co., Belknap Coal Co. \#4 (oil prod) } \\
\text { TE\&L Co. Suv. \#23, } 478 \mathrm{ft} \text { FNL \& } 150 \mathrm{ft} F W L .332 \mathrm{ft} N \text { of \#1 }\end{array}$ & & 663 & $4 / 4 / 1935$ & $4 / 11 / 1935$ & $\begin{array}{l}\text { Sh, blk: } 122 \\
\text { Sh, blk: } 270 \\
\text { Sh, blk: } 280 \\
\text { Sh, blk: } 643\end{array}$ \\
\hline 82 & $\begin{array}{l}\text { 0.T. Anderson Oil Co., Belknap Coal Co. \#5 (dry hole) } \\
\text { TE\&L Co. Suv. \#23,45.3 Ac., } 238 \mathrm{ft} \mathrm{FNL} \mathrm{\&} 150 \mathrm{ft} F W L \\
328 \mathrm{ft} \text { of } \# 4\end{array}$ & & 725 & 4/5/1935 & 4/9/1935 & $\begin{array}{l}\text { Sh, blk: } 263 \\
\text { coal: } 345 \\
\text { Sh, blk: } 660\end{array}$ \\
\hline 83 & $\begin{array}{l}\text { 0.T. Anderson \& E. W. Hunt, E.W. Hunt \#1 (dry hole) } \\
\text { TE\&L Co. Suv. Sec. } 240,160 \text { Acres, } 510 \mathrm{ft} \text { FWL \& } 150 \mathrm{ft} \text { FNL } \\
\text { N } 1 / 2 \text { of Suv. }\end{array}$ & & 1083 & 11/6/1936 & $11 / 15 / 1936$ & coal, hard: 601 \\
\hline 84 & $\begin{array}{l}\text { Rhea Anderson, Mrs. A.A. Bernhardt \#1 (dry hole) } \\
\text { TE\&L Suv., Suv. 238, } 120 \text { Ac. Tract, } 150 \mathrm{ft} \text { fr S \& E lines. }\end{array}$ & & 1028 & 9/15/1939 & 9/30/1939 & Sh, blk: 994 \\
\hline 85 & $\begin{array}{l}\text { Rhea Anderson, V.W. Kunkel \#1 (dry hole) } \\
\text { TE\&L Co. Suv., Blk } 1174,150 \text { ft each way fr SW corner of } \\
\text { Kunker Land \& was an offset to GT\&W Right of Way. }\end{array}$ & & 1202 & 4/26/1933 & $5 / 14 / 1933$ & $\begin{array}{l}\text { Sh, blk: } 570 \\
\text { Sh, blk: } 788 \\
\text { Sh, dark: } 900 \\
\text { Sh, blk: } 1170 \\
\text { Sh, blk: } 1175\end{array}$ \\
\hline 86 & $\begin{array}{l}\text { Rhea Anderson, J.P. Mullinax \#1 (dry hole) } \\
\text { TE\&L Co. Suv., Blk } 168 \text {, Ab. } 415,150 \mathrm{ftt} 470 \mathrm{ft} \mathrm{FNL,} \\
150 \mathrm{ft} F \mathrm{FL} \text { of } \mathrm{S} 400 \mathrm{Ac} \text {. of N } 80 \mathrm{Ac} \text {. Of W } 120 \mathrm{Ac} \text {. }\end{array}$ & & 1001 & 1/22/1940 & $1 / 30 / 1940$ & $\begin{array}{l}\text { Sh, blk: } 600 \\
\text { Sh, blk, sdy: } 920\end{array}$ \\
\hline 87 & $\begin{array}{l}\text { Anderson Oil Account, B.W. King \#3 (abd dry hole) } \\
\text { TE\&L Survey Sec. } 730 \text { Abst. } 735,320 \text { ac., } 1380 \mathrm{ft} \text { FEL \& } \\
1280 \mathrm{ft} \text { FSL of Surv Sec } 730,7.5 \mathrm{mi} \text { SW fr New Castle }\end{array}$ & & 4700 & $7 / 26 / 1957$ & $\begin{array}{l}8 / 10 / 1957 \\
\text { abd } 8 / 14 / 57\end{array}$ & coal: 1231 \\
\hline 88 & $\begin{array}{l}\text { Anderson Oil, Ltd. \& Assocites, Dorian-King "A" } \$ \text { " (oil prod) } \\
\text { TE\&\&L Abst. } 292 \text {, Sec } 16,320 \text { ac., } 450 \mathrm{ft} \text { FSL \& } 1000 \mathrm{ft} \text { FWL }\end{array}$ & & 3955 & $3 / 22 / 1956$ & 3/31/1956 & coal: 1050 \\
\hline 89 & $\begin{array}{l}\text { F.M. Andrews, Hearne \#1 (dry hole) } \\
\text { TE\&L Co. Suv., Sec. } 355,220 \text { Ac. Tr., } 1399 \text { ft FN \& E lines }\end{array}$ & & 725 & 3/18/1935 & 4/3/1935 & $\begin{array}{c}\text { coal: } 44 \\
\text { coal: } 141 \\
\text { coal: } 169\end{array}$ \\
\hline 90 & $\begin{array}{l}\text { Anzac Oil Corp. et al., M.K. Graham \#1 (oil prod, Martin field) } \\
\text { A.E. Gesset Suv. Abs. \#1802, } 1400 \mathrm{ft} \text { fr N \& W lines }\end{array}$ & $1160 \mathrm{GL}$ & $4 / 5 / 1912$ & 3/13/1941 & 8/16/1941 & $\begin{array}{l}\text { Sh, blk: } 240 \\
\text { Sh, blk: } 1480\end{array}$ \\
\hline 91 & $\begin{array}{l}\text { Anzac Oil Corp. et al., M.K. Graham Estate \#1D (dry hole) } \\
\text { TE\&L Co. Suv., Sec. 3410, } 6200 \mathrm{ft} \text { FWL \& } 3407 \mathrm{ft} \text { FSL } \\
\text { of Frac. "C" }\end{array}$ & $1169 \mathrm{DF}$ & 2712 & $4 / 11 / 1946$ & $5 / 27 / 1946$ & Sh, blk: 1520 \\
\hline 92 & 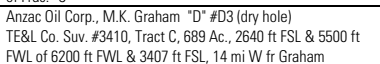 & 1201 & 4710 & $8 / 30 / 1947$ & $12 / 25 / 1947$ & Sh, blk: 1550 \\
\hline 93 & $\begin{array}{l}\text { Anzac Oil Corp. et al., M.K. Graham Est. \& E.W. Harrison \#2 } \\
\text { (oil prod); A.E. Gossett Suv., } 220 \text { Ac., } 467 \mathrm{ft} \text { FWL \& } 330 \mathrm{ft} \\
\text { FSL of Lse. }\end{array}$ & $1141 \mathrm{DF}$ & 4045 & $10 / 10 / 1944$ & $5 / 15 / 1945$ & Sh, blk: 1200 \\
\hline 94 & $\begin{array}{l}\text { Anzac Oil Corp., Graham \& Harrison \#5 (dry hole) } \\
\text { Gossett Suv. Abst. 1802, } 708.3 \text { Ac, } 330 \text { ft FSL \& } 550 \mathrm{ft} \mathrm{FEL} \\
3 \mathrm{mi} \text { W fr South Bend }\end{array}$ & $\begin{array}{l}1084 \mathrm{GL} \\
1085 \mathrm{DF}\end{array}$ & 2235 & $5 / 25 / 1951$ & 6/29/1951 & $\begin{array}{l}\text { Sh, dark: } 1155 \\
\text { Sh, dark: } 1370\end{array}$ \\
\hline 95 & $\begin{array}{l}\text { Anzzac Oil Corp. A.P. Martin \#4 (dry hole) } \\
\text { Geo. Cothran Suv., Sec. A-1401, } 330 \mathrm{ft} \mathrm{FSL} \mathrm{\&} 467 \mathrm{ft} F \mathrm{LE}, \\
4 \mathrm{mi} \text { W fr South Bend }\end{array}$ & 1173 DF & 2365 & 12/10/1946 & $2 / 4 / 1947$ & Sh, blk: 255 \\
\hline 96 & $\begin{array}{l}\text { Anzac Oil Corp. et al., A.P. Martin \#7 (oil prod) } \\
\text { Cothran Sur., Abs. } 1401,148.3 \text { ac., } 905 \mathrm{ft} F W L \text { \& } 1060 \mathrm{ft} \mathrm{FNL}\end{array}$ & $\begin{array}{l}1170 \mathrm{GL} \\
172 \mathrm{DF}\end{array}$ & 2216 & 1/11/1952 & 2/19/1952 & $\begin{array}{l}\text { Sh, dark: } 255 \\
\text { Sh, blk: } 1280\end{array}$ \\
\hline 97 & $\begin{array}{l}\text { Anzac Oil Corp. et al., A.P. Martin \#8 (oil prod) } \\
\text { Geo. Cothran Suv.. Abst } 1401,148.3 \text { ac. } 400 \mathrm{ft} F N L \& \\
1220 \mathrm{ft} F \mathrm{FW} ; 660 \mathrm{ft} \mathrm{N} \& 315 \mathrm{ft} E \text { of } \# 7,4.5 \mathrm{mi} \text { W of } S \text { Bend }\end{array}$ & $\begin{array}{l}1172 \mathrm{GL} \\
1174 \mathrm{DF}\end{array}$ & 2372 & $4 / 26 / 1952$ & $5 / 25 / 1952$ & $\begin{array}{l}\text { Sh, dark: } 257 \\
\text { Sh, dark: } 2165 \\
\text { Sh, dark: } 1505\end{array}$ \\
\hline 98 & 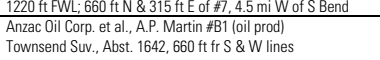 & $\begin{array}{l}1187 \mathrm{GL} \\
1192 \mathrm{RT}\end{array}$ & 2403 & $12 / 15 / 1942$ & $12 / 19 / 1942$ & $\begin{array}{l}\text { Sh, adar.: } 1505 \\
\text { Sh, blk: } 1768\end{array}$ \\
\hline
\end{tabular}




\begin{tabular}{|c|c|c|c|c|c|c|}
\hline $\begin{array}{l}\text { Occurrence } \\
\text { No. }\end{array}$ & $\begin{array}{l}\text { Well name / } \\
\text { location }\end{array}$ & $\begin{array}{c}\text { Elevation } \\
\text { (ft) }\end{array}$ & $\begin{array}{c}\text { Total } \\
\text { depth (ft) }\end{array}$ & $\begin{array}{l}\text { Spud } \\
\text { date }\end{array}$ & $\begin{array}{c}\text { Compl; PB } \\
\text { dates }\end{array}$ & $\begin{array}{l}\text { Coal and black or dark-gray shale } \\
\text { occurrences (depth in ft) }\end{array}$ \\
\hline 99 & $\begin{array}{l}\text { Townsend Suv., Abst. 1642, } 80 \text { Ac. Tr., } 8008 \mathrm{ft} \text { fr N \& } \\
330 \mathrm{fft} \text { fr WL; } 4 \text { mi W fr South Bend }\end{array}$ & $1208 \mathrm{DF}$ & 2206 & 4/10/1951 & 5/11/1951 & $\begin{array}{l}\text { Sh, dark: } 327 \\
\text { Sh, dark: } 393 \\
\text { Sh, dark: } 565 \\
\text { Sh, blk: } 1345 \\
\text { Sh, dark: } 1570\end{array}$ \\
\hline 100 & $\begin{array}{l}\text { Arcadia Refining Co., Jeffery \#1 (dry hole) } \\
\text { TE\&L Co. Suv., Sec } 47,150 \mathrm{ft} F E L \text { \& } 150 \mathrm{FSL} N 40 \mathrm{Ac} \\
\text { of E } 80 \mathrm{Ac} \text { of } \mathrm{S} 160 \mathrm{Ac} \text { of Suv } \# 47\end{array}$ & & 898 & $11 / 1 / 1930$ & 12/1/1930 & $\begin{array}{l}\text { Sh, blk: } 230 \\
\text { Sh, blk: } 375 \\
\text { Sh, blk: } 436 \\
\text { Sh, blk: } 445 \\
\text { Sh, blk: } 453 \\
\text { Sh, blk: } 761 \\
\text { Sh, blk: } 826\end{array}$ \\
\hline 101 & $\begin{array}{l}\text { Archer Drilling and Producing Company, Evans \#1 (dry hole) } \\
\text { 1. Holman Sur } 55 \text { Acres } 150 \mathrm{ft} \mathrm{FLL} \mathrm{\&} 450 \mathrm{ft} \mathrm{FWL} \mathrm{of} \mathrm{Lse,} \\
W 55 \text { ac out of } 160 \text { ac of Sur; } 5 \text { mi NW fr Olney }\end{array}$ & & 1339 & $1 / 27 / 1954$ & 1/28/1954 & $\begin{array}{c}\text { coal streaks: } 903.5 \\
\text { coal: } 912 \\
\text { Sh, blk: } 1287 \\
\text { Sh, blk: } 1312.5 \\
\end{array}$ \\
\hline 102 & $\begin{array}{l}\text { Archer Drilling and Prod. Comp., Mary Wilson \#2 (oil prod) } \\
\text { I. Hollman Sur., } 60 \text { ac., } 300 \mathrm{ft} \text { FSL \& } 1050 \mathrm{ft} \text { FEL }\end{array}$ & & 1239 & 11//7/1955 & 11/8/1955 & Sh, blk: 1237 \\
\hline 103 & $\begin{array}{l}\text { Floyd Ard, Jr., Belknap Coal Co \#1 (dry hole) } \\
\text { TE\&LL Suv. Bik } 21,150 \mathrm{ft} \text { FNL \& } 150 \mathrm{ft} \mathrm{FWL,} \mathrm{W} 20 \text { Ac. } \\
\text { N } 40 \text { Ac. SW } 1 / 4 \text { Blk } 21\end{array}$ & & 800 & $12 / 28 / 1930$ & 1/20/1931 & $\begin{array}{l}\text { coal: } 100 \\
\text { Sh, blk: } 160 \\
\text { Sh, blk: } 253\end{array}$ \\
\hline 104 & $\begin{array}{l}\text { Floyd Ard, Jr., Belknap Coal Co \#1 (dry hole) } \\
\text { TE\&L Co. Suv., Blk 21, } 150 \text { ft each way fr NE Cor. } \\
\text { SE } 20 \text { Ac. of SW 1/4 Blk } 21\end{array}$ & & 745 & $12 / 20 / 1932$ & $12 / 31 / 1932$ & $\begin{array}{l}\text { Sh, blk: } 247 \\
\text { Sh, blk: } 287 \\
\text { Sh, blk: } 295 \\
\text { Sh, blk: } 305\end{array}$ \\
\hline 105 & $\begin{array}{l}\text { Floyd Ard, Jr., Belknap Coal Co \#2 (oil prod) } \\
\text { TE\&L Suv. Blk } 22\end{array}$ & & 722 & 5/15/1931 & $6 / 1 / 1931$ & Sh, dark: 704 \\
\hline 106 & $\begin{array}{l}\text { Floyd A Ard, J., Jennie Jeffrey \#D1 (dry hole) } \\
\text { TE\&L Suv. Blk } 48,1083 \mathrm{ft} S \& 150 \mathrm{ft} W \mathrm{fr} \text { NE corner }\end{array}$ & 1216 & 900 & 1/10/1932 & $2 / 1 / 1932$ & $\begin{array}{l}\text { coal: } 300 \\
\text { Sh, blk: } 432 \\
\text { Sh, blk: } 816\end{array}$ \\
\hline 107 & $\begin{array}{l}\text { Floyd Ard, Jr., Jennie Jeffrey \#F1 (dry hole) } \\
\text { TE\&L Co Survey, Blk } 48,150 \mathrm{ft} \text { each way fr NW Cor. } \\
\text { of E } 30 \mathrm{Ac} \text {. of N } 80 \mathrm{AC} \text {. }\end{array}$ & 1209 & 885 & 1/10/1932 & $2 / 1 / 1932$ & $\begin{array}{l}\text { coal: } 302 \\
\text { coal: } 426 \\
\text { coal } 460 \\
\text { Sh, blk: } 830 \\
\text { ch }\end{array}$ \\
\hline 108 & $\begin{array}{l}\text { Floyd Ard, Jr., S.R. Jefferey \#1 (dry hole) } \\
\text { TE\&L Co Survey, Blk 41, } 450 \mathrm{ft} S \& 150 \mathrm{ft} \text { E NW Cor. Blk }\end{array}$ & 1203 & 825 & 3/10/1931 & 3/23/1931 & $\begin{array}{l}\text { Sh, blk: } 235 \\
\text { coal: } 350 \\
\text { coal: } 372\end{array}$ \\
\hline 109 & $\begin{array}{l}\text { Floyd Ard, Jr., D.L. Keith et al. \#1 (dry hole) } \\
\text { TE\&L Suv. BIk 28, } 150 \mathrm{ft} \text { fr S \& E lines }\end{array}$ & 1190 & 756 & 9/23/1930 & $10 / 11 / 1930$ & $\begin{array}{l}\text { coal; Sh, blk: } 270 \\
\text { Sh, blk: } 720\end{array}$ \\
\hline 110 & $\begin{array}{l}\text { Floyd Ard, Jr., J.J. Perkins \#1 (dry hole) } \\
\text { TE\&L Co. Suv. Blk 21, } 150 \mathrm{ft} \text { \& \& E of NW Cor. E } 1 / 2 \text { of } \\
\text { SW 1/4 Blk 21 }\end{array}$ & 1200 & 900 & $3 / 28 / 1931$ & $3 / 31 / 1931$ & Sh, blk: 273 \\
\hline 111 & $\begin{array}{l}\text { Floyd Ard, Jr., Mrs. G.B. Terrell Est. \#1 (oil well) } \\
\text { TE\&L Suv. BIk 255, } 150 \text { FSL \& } 150 \mathrm{ft} \text { FWL }\end{array}$ & & 696 & $7 / 19 / 1930$ & $9 / 18 / 1930$ & $\begin{array}{l}\text { Sh, blk: } 260 \\
\text { Sh, blk: } 689\end{array}$ \\
\hline 112 & $\begin{array}{l}\text { Floyd Ard \& Pace, Belknap Coal Co. \#1 (dry hole) } \\
\text { TE\&L Co. Suv. Sec. 23, } 150 \mathrm{ft} \text { FNL \& } 150 \mathrm{ft} \mathrm{FEL} \mathrm{SE} \mathrm{1/4}\end{array}$ & & 730 & 8/25/1935 & 8/30/1935 & $\begin{array}{l}\text { Sh, blk: } 255 \\
\text { coal: } 285\end{array}$ \\
\hline 113 & $\begin{array}{l}\text { Ard Hansen et al., Belknap Coal Co. \#1 (abd dry hole) } \\
\text { TE\&L Co. Suv. Blk 21, } 40 \text { Ac., } 4 \text { mi NW fr New Castle }\end{array}$ & & 780 & $4 / 1 / 1941$ & $4 / 5 / 1941$ & Sh, blk: 729 \\
\hline 114 & $\begin{array}{l}\text { Ard-Hansen et al., Belknap Coal Co. \#1B (dry hole) } \\
\text { TE\&LL Co. Suv. Blk 23, } 40 \text { Ac., } 8 \text { mi S fr Olney }\end{array}$ & & 725 & 3/13/1941 & 3/22/1941 & $\begin{array}{l}\text { coal: } 70 \\
\text { Sh, dark: } 95 \\
\text { Sh, blk: } 250 \\
\text { Sh, blk: } 472\end{array}$ \\
\hline 115 & $\begin{array}{l}\text { Arkansas Fuel Oil Company, J.W. Bullock \#1 (dry hole) } \\
\text { TE\&LL Co. Suv. Sec. } 344,957,5 \mathrm{ft} \text { S \& } 933.3 \mathrm{ft} \text { W of } \\
\text { NE Cor. Sec. } 344 .\end{array}$ & & 893 & 9/26/1930 & 10/12/1930 & $\begin{array}{l}\text { Sh, blk: } 437 \\
\text { Sh, blk: } 897\end{array}$ \\
\hline 116 & $\begin{array}{l}\text { Arkansas Fuel Oil Company, S.R. Jeffery "A" \#1 (dry hole) } \\
\text { TE\&L Co. Blk } 49 \text {, in center of } S 150 \text { acres of Blk } 49\end{array}$ & & 905 & $8 / 6 / 1930$ & 8/16/1930 & $\begin{array}{l}\text { lignite: } 420 \\
\text { coal: } 460 \\
\text { Sh, blk: } 790\end{array}$ \\
\hline 117 & $\begin{array}{l}\text { Arkansas Fuel Oil Company, S.R. Jeffery "C" \#1 (dry hole) } \\
\text { TE\&LL Co. Suv. Sec. \#55, } 1092 \mathrm{ft} \text { \& } 934 \mathrm{ft} \text { Sof NW Cor. }\end{array}$ & & 956 & 9/14/1930 & 9/22/1930 & $\begin{array}{l}\text { Sh, blk: } 535 \\
\text { Sh, blk: } 851\end{array}$ \\
\hline 118 & $\begin{array}{l}\text { Armour Properties, Carmack \#1 (oil prod) } \\
\text { YCSL Sur. Blk 16, } 317.5 \text { ac, } 596 \text { ft FWL \& } 656 \mathrm{ft} \text { FSL of Ise. }\end{array}$ & & 3712 & $11 / 27 / 1948$ & $12 / 9 / 1948$ & $\begin{array}{c}\text { coal: } 200 \\
\text { coal: } 310\end{array}$ \\
\hline 119 & $\begin{array}{l}\text { Mel Amstrong, Mel Amstrong-Pauline Ferguson \#1 (dry hole) } \\
\text { L.W. Smith Suv. } 130 \text { Acres, Sylphia Tyne Abst } 274 \text {, } \\
150 \mathrm{ft} \mathrm{FNL} \mathrm{\&} 150 \mathrm{ft} \mathrm{FWL}\end{array}$ & & 657 & 9/12/1932 & 10/17/1932 & $\begin{array}{l}\text { Sh, blk: } 32 \\
\text { Sh, blk: } 366\end{array}$ \\
\hline 120 & $\begin{array}{l}\text { Mel Amstrong, E.M. Greathouse \#1 (dry hole) } \\
\text { Robert Barr Suv. } 120 \text { Ac., } 250 \mathrm{ft} \text { FSL \& } 600 \mathrm{ft} \mathrm{FEL}\end{array}$ & & & & 4/17/1933 & Sh, blk: 835 \\
\hline 121 & $\begin{array}{l}\text { Mel Amstrong, D.M. Spearman \& T.C. McCloud \#1 (dry hole) } \\
\text { S. Sallie Survey \#252, } 150 \mathrm{ft} \mathrm{FNL} \mathrm{\&} 150 \mathrm{ft} f \mathrm{fr} \text { corner of } \\
\text { R. Morrison lease, } 53 \text { Acres. }\end{array}$ & & 670 & $9 / 27 / 1932$ & $11 / 2 / 1932$ & $\begin{array}{l}\text { Sh, blk: } 95 \\
\text { Sh, blk: } 545\end{array}$ \\
\hline 122 & $\begin{array}{l}\text { Associated Developers, W.P. Terrell et al. \#1 (dry hole) } \\
\text { TE\&LL Co. Abst. } 532,990 \text { ft fr } \& \text { \& E lines } 320 \text { Ac Tr. } \\
\text { TE\&LL Suv \# 285, Abst } 532\end{array}$ & & 3011 & $6 / 2 / 1936$ & 6/25/1936 & $\begin{array}{l}\text { coal; shale: } 1040 \\
\text { Sh, blk: } 1048\end{array}$ \\
\hline 123 & $\begin{array}{l}\text { Associated Developers, E.G. Williamson \#1 (dry hole) } \\
\text { TE\&L Co. Suv. Blk } 601,330 \mathrm{ft} \text { FNL \& } 990 \mathrm{ft} F \mathrm{FLN} \text { N 1/2 Suv. }\end{array}$ & & 3000 & 7/23/1936 & 8/10/1936 & Sh, blk: 973 \\
\hline 124 & $\begin{array}{l}\text { AAssciated Developers, E.G. Williamson \#1 (dry hole) } \\
\text { TE\&L Co. Suv. Blk 286, } 160 \text { Acres, } 330 \mathrm{ft} F \mathrm{FL} \& 990 \mathrm{ft} F \mathrm{FS}\end{array}$ & & 3000 & $11 / 10 / 1936$ & $11 / 28 / 1936$ & $\begin{array}{l}\text { coal: } 167 \\
\text { Sh, blk: } 998 \\
\end{array}$ \\
\hline 125 & $\begin{array}{l}\text { Associates 0il Co. Pat Marr, Lisle \#1 (dry hole) } \\
\text { TE\&L Suv., } 60 \text { Ac., Sec. } 260,250 \mathrm{ft} \text { FEL \& } 450 \mathrm{ft} \text { FSL }\end{array}$ & 1193 & 767 & $7 / 4 / 1930$ & $7 / 9 / 1930$ & $\begin{array}{l}\text { Sh, dark: } 85 \\
\text { Sh, blk: } 230 \\
\text { Sh, blk: } 290\end{array}$ \\
\hline 126 & $\begin{array}{l}\text { Paul Atchley, J.P. Atchley \#1 (dry hole) } \\
\text { TE\&L Suv., Blk 226, } 80 \text { acre tract, } 1070 \mathrm{ft} \text { FEL \& } 530 \mathrm{ft} \text { FSL. }\end{array}$ & & 995 & $1 / 6 / 1937$ & 1/20/1937 & $\begin{array}{l}\text { Sh, blk: } 320 \\
\text { Sh, blk: } 252 \\
\text { Sh, dark: } 649 \\
\text { Sh, blk: } 972\end{array}$ \\
\hline 127 & $\begin{array}{l}\text { Paul Atchley, J.P. Atchley \#2 (oil prod) } \\
\text { TE\&L Co. Suv. Bik 226, } 80 \text { Acre Tract, } 940 \mathrm{ft} N \& 750 \mathrm{ft} W \\
\text { of SE Corner of Blk } 226\end{array}$ & & 1000 & $2 / 1 / 1937$ & 2/13/1937 & $\begin{array}{l}\text { Sh, blk: } 500 \\
\text { Sh, blk: } 515 \\
\text { Sh, blk: } 565\end{array}$ \\
\hline 128 & $\begin{array}{l}\text { Paul Atchley, J.P. Atchley Farm \#3 (dry hole) } \\
\text { TE\&L Co. Suv. Blk 226, } 80 \text { acre Tr., } 310 \mathrm{ft} \mathrm{NW} \text { of \#2 }\end{array}$ & & 1005 & 3/26/1937 & 4/6/1937 & $\begin{array}{l}\text { Sh, blk: } 332 \\
\text { Sh, blk: } 360 \\
\text { Sh, , blk: } 503 \\
\text { Sh, blk: } 515 \\
\text { Sh, blk: } 521 \\
\text { Sh, blk: } 544 \\
\text { Sh, blk: } 571 \\
\text { Sh, blk: } 579 \\
\text { Sh, blk: } 969 \\
\text { Sh, blk: } 990\end{array}$ \\
\hline 129 & $\begin{array}{l}\text { Paul Atchley, S.M.J. Benson \#10 (oil prod) } \\
\text { TE\&L Co. Suv. Blk } 1392,50 \text { Ac. Lse., } 750 \mathrm{ft} \text { \& } 450 \mathrm{ft} S \text { of } \\
\text { NW Cor. } 50 \text { Ac. Lse. } 300 \mathrm{ft} \text { of } \# 9 \text { well }\end{array}$ & & 947 & $2 / 8 / 1938$ & $3 / 2 / 1938$ & $\begin{array}{l}\text { Sh, blk: } 275 \\
\text { Sh, blk: } 555 \\
\text { Sh, blk: } 558 \\
\text { Sh, blk: } 896\end{array}$ \\
\hline
\end{tabular}




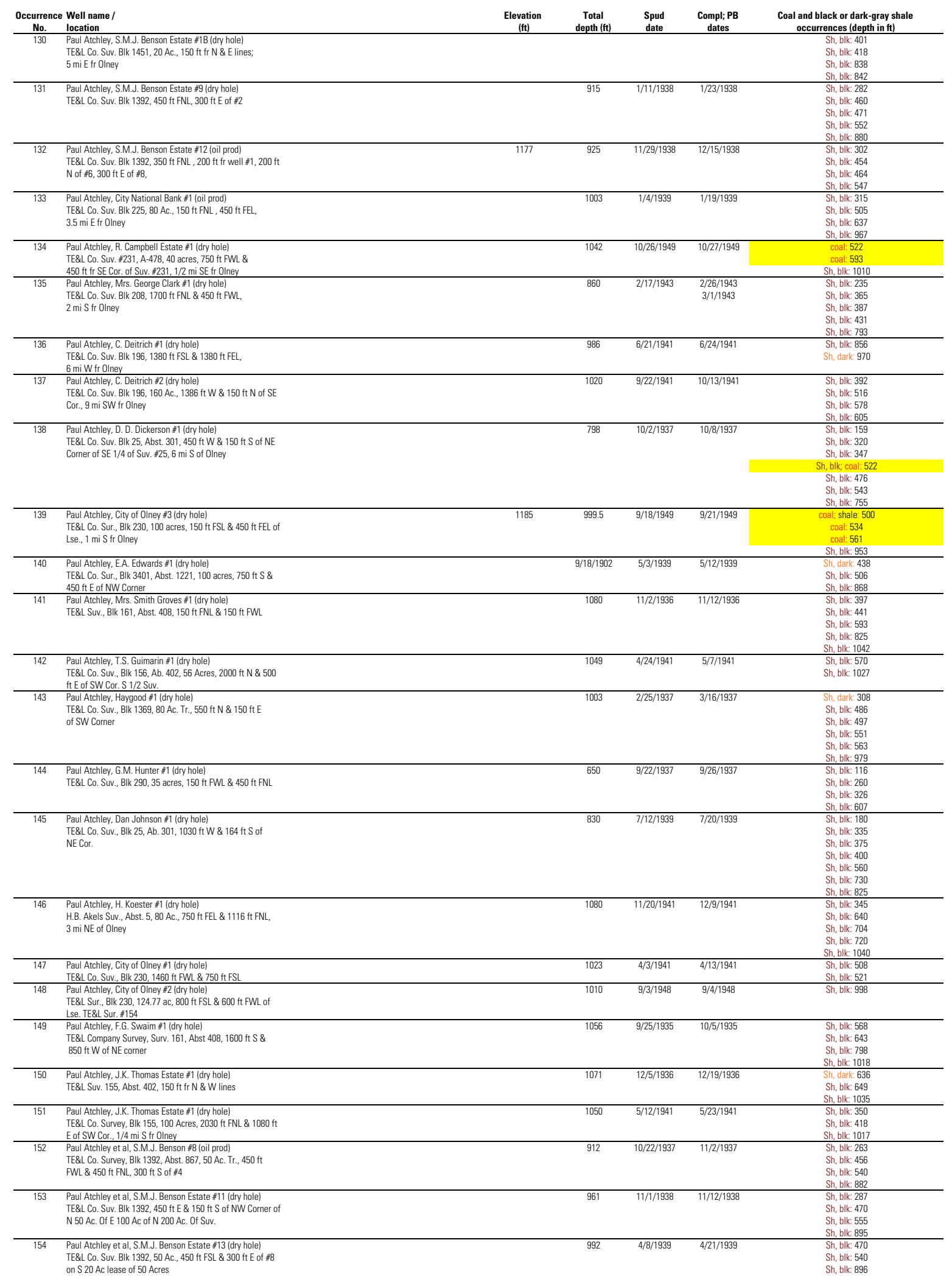




\begin{tabular}{|c|c|c|c|c|c|c|}
\hline $\begin{array}{l}\text { Occurrence } \\
\text { No. }\end{array}$ & $\begin{array}{l}\text { Well name / } \\
\text { location }\end{array}$ & $\begin{array}{c}\text { Elevation } \\
\text { (ft) }\end{array}$ & $\begin{array}{c}\text { Total } \\
\text { depth (ft) }\end{array}$ & $\begin{array}{l}\text { Spud } \\
\text { date }\end{array}$ & $\begin{array}{c}\text { Compl; PB } \\
\text { dates }\end{array}$ & $\begin{array}{l}\text { Coal and black or dark-gray shale } \\
\text { occurrences (depth in ft) }\end{array}$ \\
\hline 155 & $\begin{array}{l}\text { Atchley, Shira et al, S.M.J. Benson \#1 (oil prod) } \\
\text { TE\&L Suv. Blk } 1392,750 \mathrm{ft} \text { \& } 1350 \mathrm{ft} \text { W of NE Cor. of Suv. }\end{array}$ & & 899 & $3 / 10 / 1936$ & $3 / 19 / 1936$ & $\begin{array}{l}\text { Sh, blk: } 267 \\
\text { Sh, blk: } 460 \\
\text { Sh, blk: } 540\end{array}$ \\
\hline 156 & $\begin{array}{l}\text { Atchley, Shira et al, S.M.J.J Benson \#2 (oil prod) } \\
\text { TE\&L Suv. Blk 1392, } 150 \mathrm{ft} F W L \text { FW } 279 \mathrm{ft} N \text { of well \#1 }\end{array}$ & 1180 & 907 & $4 / 3 / 1936$ & 4/13/1936 & $\begin{array}{l}\text { Sh, blk: } 246 \\
\text { Sh, blk: } 267 \\
\text { Sh, blk: } 451 \\
\text { Sh, blk: } 458 \\
\text { Sh, blk: } 523 \\
\text { Sh, blk: } 535 \\
\text { Sh. blk: } 880\end{array}$ \\
\hline 157 & $\begin{array}{l}\text { Paul Atchley \& J.B. Shira, S.M.J. Benson \#4 (oil prod) } \\
\text { TE\&L Survey, Blk 1392, } 150 \mathrm{ft} f \mathrm{fr} \& \text { W lines }\end{array}$ & 1174 & 896 & 4/25/1936 & 5/10/1936 & $\begin{array}{l}\text { Sh, dark: } 260 \\
\text { Sh, blk: } 456 \\
\text { Sh, blk: } 537 \\
\text { Sh, blk: } 865\end{array}$ \\
\hline 158 & $\begin{array}{l}\text { Paul Atchley \& J.B. Shira, S.M.J. Benson \#f (oil prod) } \\
\text { TE\&LL Survey, BBk 1392, } 150 \mathrm{ft} \text { FSL, } 400 \mathrm{ft} \text { Eof } \# 1\end{array}$ & & 948 & $5 / 8 / 1936$ & 6/6/1936 & $\begin{array}{l}\text { Sh, blk: } 271 \\
\text { Sh, dark: } 456 \\
\text { Sh, blk: } 464 \\
\text { Sh, blk: } 545 \\
\text { Sh, blk: } 887\end{array}$ \\
\hline 159 & $\begin{array}{l}\text { Atchley \& Shira et al., , S.M.J. Benson \#7 (dry hole) } \\
\text { TE\&L Co. Suv., } 30 \text { Acr., } 721 \mathrm{ft} \mathrm{S} \& 150 \mathrm{ft} \mathrm{N} \text { of E Cor. } \\
\text { of Suv. } 1392\end{array}$ & & 980 & $6 / 4 / 1936$ & $7 / 2 / 1936$ & $\begin{array}{l}\text { Sh, blk: } 268 \\
\text { Sh, blk: } 299 \\
\text { Sh, blk: } 445 \\
\text { Sh, blk: } 473 \\
\text { Sh, blk: } 553 \\
\text { Sh, blk: } 899\end{array}$ \\
\hline 160 & $\begin{array}{l}\text { Atlantic Oil Producing Co., R.H. Rachuig \#A4 (oil prod) } \\
\text { BBB\&C RR Suv., } 785 \mathrm{ft} \text { FEL \& } 150 \mathrm{ft} \mathrm{FNL}\end{array}$ & 1263 & 1183 & $4 / 2 / 1926$ & 4/16/1926 & $\begin{array}{l}\text { Sh; coal: } 426 \\
\text { Sh; coal: } 727\end{array}$ \\
\hline 161 & $\begin{array}{l}\text { Atlantic Oil Producing Co. et al., J.E. Keen \#1B (oil prod) } \\
\text { TE\&L Suv., Sec. } 3401,150 \mathrm{ft} \text { FNL \& } 750 \mathrm{ft} \mathrm{FWL}\end{array}$ & 1196 & 903.5 & 3/29/1930 & 4/19/1930 & $\begin{array}{l}\text { coal: } 418 \\
\text { coal: } 517\end{array}$ \\
\hline 162 & $\begin{array}{l}\text { Atlantic Oil Producing Co. et al., J.E. Keen \#2B (dry hole) } \\
\text { TE\&L Suv. \#3401, } 150 \mathrm{ft} \text { FNL \& } 156 \mathrm{ft} \text { FEL }\end{array}$ & 1186.5 & 908 & $4 / 25 / 1930$ & $5 / 13 / 1930$ & coal: 499 \\
\hline 163 & $\begin{array}{l}\text { A.U. } 2 \text { Oil Company, Gramell, Dufffy, \& Cook, S.M.J. Benson } \\
\text { Est. \#1 (dry hole); TE\&L Co. Suv. Blk 1392, } 40 \text { acres, } 150 \mathrm{ft} \\
\text { fr N \& E lines }\end{array}$ & & 1004 & $5 / 7 / 1936$ & 5/15/1936 & $\begin{array}{l}\text { Sh, blk: } 437 \\
\text { Sh, blk: } 505 \\
\text { Sh, blk: } 864\end{array}$ \\
\hline 164 & $\begin{array}{l}\text { Joe K. Bailey c/o Man Drlg. Co., Susan-Sallee (B.W. King)) \#1 } \\
\text { (dry hole); Susan-Sallee Sur, A-252, } 214.8 \text { Ac, } 3817 \text { ft FNL, } \\
3460 \text { ft FEL of Sec A-252 (S-252), } 3 \text { mi NE fr Loving }\end{array}$ & & 3700 & $8 / 2 / 1952$ & 8/18/1952 & coal strks: 1040 \\
\hline 165 & $\begin{array}{l}\text { Joe K. Bailey \& W.A. Lofton et ala., Brown, T.J. Routen \#1 } \\
\text { (dry hole); TE\&L Sur., Sec } 736, \text { A-471, } 80 \text { Ac., } 330 \mathrm{ft} \text { FNL } \\
\& 1650 \mathrm{ft} \text { FEL, } 10 \mathrm{mi} \text { SW of Newcastle }\end{array}$ & & 4003 & 10/14/1955 & 10/24/1955 & coal strks: 180 \\
\hline 166 & $\begin{array}{l}\text { Joe K. Bailey \& W.A. Lofton et al., B.W. King \#2 (oil prod) } \\
\text { TE\&L Sur., Blk 735, Abst } 740,80 \text { ac., } 330 \mathrm{ftt} \text { FN\&EL }\end{array}$ & & 3600 & 9/29/1955 & 10/9/1955 & $\begin{array}{l}\text { coal strks: } 326 \\
\text { coal strks: } 369\end{array}$ \\
\hline 167 & $\begin{array}{l}\text { Joe K. Bailey \& W.A. Loftton et al., B.W. King \#3 (oil prod) } \\
\text { TE\&L Sur., Blk A-740, Sec. } 735,80 \text { ac., } 330 \mathrm{ft} F \mathrm{FL} \& \text { \& } \\
625 \mathrm{ft} F \mathrm{FL}\end{array}$ & & 3603 & $5 / 7 / 1956$ & $5 / 15 / 1956$ & coal strks: 230 \\
\hline 168 & $\begin{array}{l}\text { Joe K. Bailey \& W.A. Lofton et al., E.C. Stovall \#1 (dry hole) } \\
\text { TE\&LL Sur., Blk } 715,60 \text { Ac, } 330 \mathrm{ft} \mathrm{FN \&} \mathrm{WL} \mathrm{of} \mathrm{Ise.}\end{array}$ & & 3755 & 6/1/1956 & 6/10/1956 & $\begin{array}{c}\text { coal: } 220 \\
\text { coal: } 296 \\
\text { shale; coal: } 390\end{array}$ \\
\hline 169 & $\begin{array}{l}\text { Joe K. Bailey \& J.C. Man, Jr., T. Bagley \#1 (oil prod) } \\
\text { L.W. Ross Suv., Abst. \#1352, } 44 \text { Ac., } 330 \text { ft FS \& WL of Ise }\end{array}$ & & 3627 & 3/27/1951 & $4 / 7 / 1951$ & coal; shale: 338 \\
\hline 170 & $\begin{array}{l}\text { James Blair Baker, M.M.Burris \#8 (dry hole) } \\
\text { TE\&L Co. Surv., Sec \#1365, } 40 \text { Ac, } 150 \mathrm{ft} W \& 275 \mathrm{ft} \mathrm{N} \\
\text { of SE Cor. of N } 40 \text { Ac., S } 80 \text { Ac., W } 160 \text { Ac of Sect } 1365\end{array}$ & & 698 & $6 / 7 / 1940$ & $7 / 1 / 1940$ & $\begin{array}{l}\text { coal: } 235 \\
\text { Sh, blk: } 430 \\
\text { Sh, blk: } 687\end{array}$ \\
\hline 171 & $\begin{array}{l}\text { James Blair Baker, Eula Thompson \#1 (oil prod) } \\
\text { TE\&L Suv., Blk } 2692,146 \text { ac., } 1200 \mathrm{ft} \text { FSEL \& } 990 \mathrm{ft} \text { FNEL }\end{array}$ & & 2212 & 12/6/1958 & 12/16/1958 & coal: 362 \\
\hline 172 & $\begin{array}{l}\text { James Blair Baker, D.L. Wolf \#3 (oil prod) } \\
\text { TE\&L Suv. } 296 \& 297 \mathrm{BIk} 297, \text { Abst. \#944, } 270 \text { ac., } 450 \mathrm{ft} \\
\text { FNL, } 1260 \mathrm{ft} \text { FEL of Lse, } 300 \mathrm{ft} \mathrm{S} \text { of \#2, } 5 \mathrm{mi} \text { SE fr Olney }\end{array}$ & $1170 \mathrm{GL}$ & 692 & 3/21/1947 & 3/28/1947 & $\begin{array}{c}\text { Sh, blk: } 668 \\
\text { Sh, blk; coal: } 674\end{array}$ \\
\hline 173 & $\begin{array}{l}\text { James Blair Baker, D.L. Wolf \#4 (oil prod) } \\
\text { TE\&L Suv. \#2966 \& 297, Bik } 297, \text { Abst. \#944, } 270 \text { ac.450 ft } \\
\text { FNL \& 2174 ft FWL of Suv. \# } 297,300 \mathrm{ft} \text { of } \# 3 .\end{array}$ & $1173 \mathrm{GL}$ & 700 & 3/30/1947 & 4/17/1947 & shale; coal: 317 \\
\hline 174 & $\begin{array}{l}\text { James Blair Baker et al., D.L. Wolf \#6 (dry hole) } \\
\text { TE\&L Suv. \#296 \& 297, Blk 297, Abst. \#944, } 270 \text { ac., } 715 \mathrm{ft} \\
\text { FNL \& } 2024 \mathrm{ft} \text { FWL of Suv. \# } 297,5 \mathrm{mi} \text { SE fr Olney }\end{array}$ & $1175 \mathrm{GL}$ & 696 & $4 / 3 / 1947$ & $4 / 12 / 1947$ & $\begin{array}{c}\text { coal; hd sand: } 304 \\
\text { coal; Sh, blk; Im: } 677.5\end{array}$ \\
\hline 175 & $\begin{array}{l}\text { James Blair Baker et al., D.L. Wolf \#8 (dry hole) } \\
\text { TE\&L Suv. \#296 \& } 297 \text {, Abst. } 944,9708 \mathrm{ft} \text { FNL \& } 1865 \mathrm{ft} \\
\text { FWL of Blk } 297\end{array}$ & $\begin{array}{c}\text { KB depths } \\
3.5 \mathrm{ft} \text { above } \\
\mathrm{GL}\end{array}$ & 831 & $4 / 7 / 1947$ & 4/10/1947 & $\begin{array}{l}\text { Sh, blk; coal: } 681 \\
\text { Sh, blk: } 728 \\
\text { Sh, blk; coal: } 775\end{array}$ \\
\hline 176 & $\begin{array}{l}\text { James Blair Baker et al., D.L. Wolf \#B1 (dry hole) } \\
\text { TE\&L Co Suv. \#296, 270 ac, } 1150 \mathrm{ft} \text { FNL \& } 650 \mathrm{ft} F \mathrm{WL} \\
\text { of Blk } 296,5 \mathrm{mi} \text { SE fm Olney }\end{array}$ & & 752 & 6/30/1949 & 7/1/1949 & $\begin{array}{c}\text { coal: } 348 \\
\text { coal; sh: } 695\end{array}$ \\
\hline 177 & $\begin{array}{l}\text { James Blair Baker et al., H.M. Donnell \#1B (oil prod) } \\
\text { W.L. Donnel Pre-Emp. Abst 1588, } 120 \text { Ac. Tr., } 250 \mathrm{ft} \mathrm{FWL} \\
\& 250 \mathrm{ft} \text { FSL }\end{array}$ & 1121 & $\begin{array}{r}3304.5 \\
3302 \mathrm{~PB}\end{array}$ & 6/18/1934 & 8/15/19334 & $\begin{array}{l}\text { Sh ("slate"), blk: } 330 \\
\text { Sh, blk: } 592 \\
\text { Sh ("slate"), dark: } 1558 \\
\end{array}$ \\
\hline 178 & $\begin{array}{l}\text { James Blair Baker et al., H.M. Donnell \#2 (oil prod) } \\
\text { W.L. Donnel Suv. S.P. Ry Co. SW 1/4 Sec. 4, Abst. 1830, } \\
2000 \mathrm{ft} \text { FWL \& } 200 \mathrm{ft} F \mathrm{FL} \text {. }\end{array}$ & 1146 & 3332 & $11 / 30 / 1929$ & $1 / 12 / 1930$ & Sh, blk: $0-12$ \\
\hline 179 & $\begin{array}{l}\text { James Blair Baker, Jr., 0.S. Perkins \#1 (oil prod) } \\
\text { TE\&L Co Suv. Sec. } 206,120 \text { ac, } 150 \mathrm{ft} \text { FS \& E lines } \\
\text { ("1086 ft W \& } 151 \mathrm{ft} \mathrm{N} \text { of SE Cor. of } 160 \text { Ac. in Blk } 206 " \text { in } \\
\text { separate, typed record) }\end{array}$ & 1207 & 848 & 12/5/1933 & 8/31/1934 & $\begin{array}{l}\text { Sh, blk: } 190 \\
\text { Sh, blk: } 363 \\
\text { Sh, blk: } 395 \\
\text { Sh, blk: } 439 \\
\text { Sh, blk: } 722 \\
\end{array}$ \\
\hline 180 & $\begin{array}{l}\text { Baker \& Camp, Trustee, G.R. Smith \#2 (dry hole) } \\
\text { TE\&L Co Suv. Blk 1365, } 40 \text { AC., } 150 \mathrm{ft} \text { FS \& E lines of lease }\end{array}$ & 1181 & 772 & 11/6/1933 & $11 / 16 / 1933$ & $\begin{array}{l}\text { Sh, blk: } 198 \\
\text { Sh, blk: } 310 \\
\text { Sh, blk: } 334 \\
\text { Sh, blk: } 356 \\
\text { Sh, blk: } 400 \\
\text { Sh, blk: } 702\end{array}$ \\
\hline 181 & $\begin{array}{l}\text { Baker \& Camp Trustees, G.R. Smith et al., \#3 (oil prod) } \\
\text { TE\&L Co Suv. Sec. 1365, } 150 \mathrm{ft} \text { FWL \& } 450 \mathrm{ft} \text { FSL }\end{array}$ & 1182 & $\begin{array}{c}706 \\
695 \mathrm{~PB}\end{array}$ & $8 / 2 / 1934$ & 8/15/1934 & $\begin{array}{l}\text { Sh, blk: } 160 \\
\text { Sh, blk: } 334 \\
\text { Sh, blk: } 398 \\
\text { Sh, blk: } 535 \\
\end{array}$ \\
\hline 182 & $\begin{array}{l}\text { Baker \& Camp Trustees, G.R. Smith et al., \#4 (dry hole) } \\
\text { TE\&L Co Suv. Blk } 1365,40 \text { Ac., } 150 \mathrm{ft} F W L \text { \& } 750 \mathrm{ft} \mathrm{FSL}, \\
300 \mathrm{ft} \text { N of \#3 }\end{array}$ & & 895 & 4/25/1938 & $5 / 5 / 1938$ & $\begin{array}{l}\text { Sh, blk: } 163 \\
\text { Sh, blk: } 257 \\
\text { Sh, blk: } 320 \\
\text { Sh, blk: } 333 \\
\text { Sh, blk: } 366 \\
\text { Sh, blk: } 395 \\
\text { Sh, blk: } 702 \\
\end{array}$ \\
\hline 183 & $\begin{array}{l}\text { Baker Oils, Inc., 0.S. Perkins \#2 (oil prod) } \\
\text { TE\&L Co. Suv. Blk 206, Abst. } 453,150 \mathrm{ft} \text { FNL \& } 150 \mathrm{ft} \text { FEL }\end{array}$ & 1209.5 & 739 & $1 / 7 / 1935$ & 1/13/1935 & $\begin{array}{l}\text { Sh, blk: } 205 \\
\text { Sh, blk: } 365 \\
\text { Sh, blk: } 380 \\
\text { Sh, blk: } 730\end{array}$ \\
\hline
\end{tabular}




\begin{tabular}{|c|c|}
\hline $\begin{array}{l}\text { Occurrence } \\
\text { No. }\end{array}$ & $\begin{array}{l}\text { Well name / } \\
\text { location }\end{array}$ \\
\hline 184 & Baker Oils, Inc., 0.S. Perkins \#3 (oil prod) \\
\hline & TE\&L Co. Suv. Blk 206, Abst. 453, 60 Acres, 150 ft FEL \& \\
\hline & $450 \mathrm{ft} \mathrm{FNL,} 350 \mathrm{ft} \mathrm{S}$ of $\# 2$ \\
\hline & \\
\hline & \\
\hline & \\
\hline & \\
\hline & \\
\hline 185 & Baker Oils, Inc., 0.S. Perkins \#6 (oil prod) \\
\hline & TE\&L Co. Suv. Blk 206, $450 \mathrm{ft}$ fr N \& E lines \\
\hline & \\
\hline 186 & Barker \& Pardue, H.M. Donnell \#1 (dry hole) \\
\hline & Wm. Stinnott Suv., Abst. 1754, $150 \mathrm{ft}$ fr SL \& EL \\
\hline 187 & Baker Oils, Inc., O.S. Perkins \#4 (oil prod) \\
\hline & TE\&L Co. Suv. Blk 206, $450 \mathrm{ft} \mathrm{FEL} \mathrm{\&} 150 \mathrm{ft} \mathrm{FNL}$ \\
\hline & \\
\hline & \\
\hline & \\
\hline 188 & $\begin{array}{l}\text { Baker \& Winfrey, Grigsby \#1 (dry hole) } \\
\text { TE\&L Co } 415,320 \mathrm{Ac} \text {. } 50 \text { ftFWL \& } 1050 \mathrm{ftEN}\end{array}$ \\
\hline 189 & J.W. Baldwin, J.C. Mann, J., UOE Bailey, J.J. Engles \#1 \\
\hline & $\begin{array}{l}\text { (dry hole), TE\&L Sur. A-785, } 33 \text { acre, } 330 \mathrm{ft} \text { f } S \& W \text { Ins of } \\
\text { Sec } 627,0.5 \mathrm{mi} \text { SE fr Jean }\end{array}$ \\
\hline 190 & Ball Dil \& Gas Co et al., B.R. Jeske \#1 (dry hole) \\
\hline & TE\&L Co. Surv., Blk 239,142 ac. tr., $450 \mathrm{ft} \mathrm{FEL} \mathrm{\&} 750 \mathrm{ft}$ FNL \\
\hline 191 & Ben Banner, Fich Estate \#1 (dry hole) \\
\hline & TE\&L Co. Suv. Sec. \#40, $150 \mathrm{ft}$ each way fr NE Cor. \\
\hline & SW $1 / 4 \operatorname{Sec} 40$ \\
\hline & \\
\hline 192 & Ben Banner, Jeffery Estate \#1 (dry hole) \\
\hline & TE\&L Co. Suv. Sec. \#47, $450 \mathrm{ft} \mathrm{FNL} \mathrm{\&} 150 \mathrm{ft} \mathrm{FWL} \mathrm{SE1/4} \mathrm{Sec.}$ \\
\hline & \\
\hline 193 & Ben Banner, Jeffery Estate \#1 (dry hole) \\
\hline & TE\&L C. Suv. Sec. $51,150 \mathrm{ft}$ each way out of SW Cor. \\
\hline & SE $1 / 4$ Sec 51 \\
\hline 194 & Ben Banner, J.K. Jeffery \#1 (dry hole) \\
\hline & $\begin{array}{l}\text { TE\&L Co. Suv. Sec. } 48,1000 \mathrm{ft} F S L \& 1900 \mathrm{ft} \text { W of E line } \\
\text { of Sec. } 48\end{array}$ \\
\hline 195 & Ben Banner, J.K. Jeffery \#1 (dry hole) \\
\hline & TE\&L Co. Suv. Sec. $55,933 \mathrm{ft} N$ of S line \& $1867 \mathrm{ft}$ E of \\
\hline & W line \\
\hline 196 & Ben Banner, J.K. Jeffery \#1 (dry hole) \\
\hline & TE\&L Co. Suv. Sec. $57,933 \mathrm{ft} \mathrm{W}$ of $E$ line \& $466 \mathrm{ft} N$ of $S$ line \\
\hline & \\
\hline & \\
\hline 197 & Ben Banner, J.K. Perkins \#1 (dry hole) \\
\hline & TE\&L Co. Suv. Sec. 23, $150 \mathrm{ft}$ FSL \& $450 \mathrm{ft}$ FWL \\
\hline & \\
\hline & \\
\hline & \\
\hline 198 & Ben Banner, J.J. Perkins \#1B (dry hole) \\
\hline & TE\&L Co. Suv. Sec. $23,150 \mathrm{ft} \mathrm{FEL} \mathrm{\&} 150 \mathrm{ft} \mathrm{FSL} \mathrm{N1/2} \mathrm{of}$ \\
\hline & $\mathrm{NW} 1 / 4$ \\
\hline 199 & Ben Banner, J.J. Perkins \#C-1 (dry hole) \\
\hline & TE\&L Co. Suv. Sec. 23, $150 \mathrm{ft}$ FEL \& $1143 \mathrm{ft} \mathrm{FNL}$ \\
\hline & \\
\hline & \\
\hline & \\
\hline 200 & Roy C. Barton, C.E. Speer \#1 (dry hole) \\
\hline & W. Stoneman Suv. Blk 3, 40 acre tract, $150 \mathrm{ft} f \mathrm{fr}$ \\
\hline & $S \&$ Elines \\
\hline & \\
\hline 201 & T.J. Bass \& C.C. Rasmussen, E.L. Arnold \#1 \\
\hline & TE\&L Co. Suv. Abst 1123, Sec 2445, $150 \mathrm{ft}$ FNEL \& $1655 \mathrm{ft}$ \\
\hline & FEL of Sur., $10 \mathrm{mi} W \mathrm{fr}$ Olney \\
\hline & L. Baum \& K. D. Lehmer, W.S. Husted \#1 (dry hole) \\
\hline 202 & S. Harrison A-142, $450 \mathrm{ft}$ FEL \& $750 \mathrm{ft} \mathrm{FNL}$ \\
\hline & \\
\hline & \\
\hline 203 & The Bay Pet. Corp., J.H. Nantz\#1 \\
\hline & BBB \& C RR Co A-29 Surv., Sec 6, 301 Acres, $2380 \mathrm{ft}$ \\
\hline & FEL of Lse. \& $660 \mathrm{ft}$ FSEL of Suv \#6 \\
\hline 204 & The Bay Pet. Corp. \& B.H. Rankin, A.L. Reese \#1 \\
\hline & (dry hole); TE\&L Co. Suv. A-668, Sec 461, 100 acres, $330 \mathrm{ft}$ \\
\hline 200 & FSL \& $1000 \mathrm{ft}$ FEL of Ise \\
\hline 205 & Bolin \& Lindsay, D.L. Wolf f1 (oil prod) \\
\hline & $\begin{array}{l}\text { TE\&L Co. Suv. Blk } 165,30 \text { Ac., } 150 \text { ft FNL \& } 150 \text { ft FWL } \\
\text { Bolin-Lindsay-Tidwell \& Hinson, G.M. James \#5 (oil prod) }\end{array}$ \\
\hline & TE\&L Co. Suv. BIK 457, Sec. A 664, 80 Ac., $330 \mathrm{ft} \mathrm{FEL} \mathrm{\&}$ \\
\hline & $1050 \mathrm{ft} F \mathrm{FL}$ \\
\hline 206 & D.H. Bolin \&/or J.J. Lynn, H.C. Gilmore \#1 (dry hole) \\
\hline & BBB \& Cry Co. Sur. A-25, Sec. 1, 2 Acr., 1650 ft FNEL \& \\
\hline 207 & 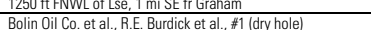 \\
\hline & TE\&L Co. Sur., Sec. 2714, 200 Ac., $300 \mathrm{ft}$ fr NL \& WL \\
\hline 208 & $\begin{array}{l}\text { of Lse, } 3 \text { mi SE fr Loving } \\
\text { Bolin Oil Co.. E. Taylor B \#1B (dry hole) }\end{array}$ \\
\hline & $\begin{array}{l}\text { Bolin Oil Co., E. Taylor B\# \#B (dry hole) } \\
\text { J.A. Holt A } 2265,330 \mathrm{ft} \text { FN \& EL of B Ise., } 1 \text { mi N fr Loving }\end{array}$ \\
\hline 209 & C.W. Boller, L.F. Gregg \#10 (oil prod) \\
\hline & A. Rohus Sur., $300 \mathrm{ft} f \mathrm{fr} \#, 300 \mathrm{ft} \mathrm{fr} \# 4$ \\
\hline & \\
\hline & \\
\hline 210 & C.W. Boller, L.F. Gregg \#11 (dry hole) \\
\hline & A. Rohus Sur., Abst. \# 240, $450 \mathrm{ft} F \mathrm{FL} \& 1050 \mathrm{ft}$ FNL \\
\hline & \\
\hline 211 & C.W. Boller, L.F. Gregg \#12 (oil prod) \\
\hline & A. Rohus Sur., $300 \mathrm{ft} \mathrm{fr} \# 11$ \& $300 \mathrm{ft} \mathrm{fr} \# 10$ \\
\hline & \\
\hline & \\
\hline 212 & C.W. Boller, J.G. Thomas \#3 (dry hole) \\
\hline & TE\&L Co. Blk $155150 \mathrm{ft} \mathrm{FSL} \mathrm{\&} 150 \mathrm{ft} \mathrm{FWL}$ \\
\hline 213 & $\begin{array}{l}\text { C.W. Boller et al., G.T. Williams \#1 (dry hole) } \\
\text { TE \& L Co. Survey Blk } 155,150 \mathrm{ft} f \mathrm{fr} \text { \& E lines }\end{array}$ \\
\hline
\end{tabular}




\begin{tabular}{|c|c|c|c|c|c|c|}
\hline $\begin{array}{l}\text { Occurrence } \\
\text { No. }\end{array}$ & $\begin{array}{l}\text { Well name / } \\
\text { location }\end{array}$ & $\begin{array}{c}\text { Elevation } \\
\quad(\mathrm{ft})\end{array}$ & $\begin{array}{c}\text { Total } \\
\text { depth (ft) }\end{array}$ & $\begin{array}{l}\text { Spud } \\
\text { date }\end{array}$ & $\begin{array}{c}\text { Compl; PB } \\
\text { dates }\end{array}$ & $\begin{array}{l}\text { Coal and black or dark-gray shale } \\
\text { occurrences (depth in ft) }\end{array}$ \\
\hline 214 & $\begin{array}{l}\text { F. W. Beardman et al, J.J. Porkins \#1 (dry hole) } \\
\text { TE\&L \#6 Suv., Abst } 847,450 \mathrm{ft} \& 150 \mathrm{ft} \text { W of NE Corn. } 40 \text { Ac. }\end{array}$ & & 738 & $12 / 20 / 1930$ & $12 / 29 / 1930$ & Sh, blk: 632 \\
\hline 215 & $\begin{array}{l}\text { F. W. Beardman et al, Baldwin Estate \#1 (dry hole) } \\
\text { TE\&L RR Suv. Sec. } 20,150 \mathrm{ft} F N L \& 450 \mathrm{ft} \text { FEL }\end{array}$ & 1194 & 752 & 8/11/1930 & $8 / 17 / 1930$ & $\begin{array}{c}\text { coal: } 256 \\
\text { Sh, blk: } 706\end{array}$ \\
\hline 216 & $\begin{array}{l}\text { Mrs. C.E. Beavers, Barnett \& Gibbs \#1 (oil prod) } \\
\text { TE\&L Suv. Blk } 2245,450 \mathrm{ft} \text { each way frm SE Corner of SW } 1 / 4 \text { of SW1/4 Blk }\end{array}$ & & 10/16/1901 & 9/17/1935 & 9/20/1935 & $\begin{array}{l}\text { Sh, blk: } 258 \\
\text { Sh, blk: } 320\end{array}$ \\
\hline 217 & $\begin{array}{l}\text { C.E. Beavers\& Son, Barnett \& Gibbs } \# 2 \text { (dry hole) } \\
\text { TE\&L Co. Suv. } 750 \mathrm{ft} \text { each way fr SE Corner of SW1/4 of Blk } 2245\end{array}$ & & 702 & 9/29/1935 & 10/3/1935 & coal: 119 \\
\hline 218 & $\begin{array}{l}\text { Mrs. C.E. Beavers, Barnett \& Gibbs \#3 (oil prod) } \\
\text { TE\&L Suv Bkk } 2245450 \mathrm{ft} F \mathrm{FL} \& \text { \& } 750 \mathrm{ft} \text { FEL of SW1/4 of Blk } 2245\end{array}$ & & 650 & 10/17/1935 & 10/21/1935 & coal: 409 \\
\hline 219 & $\begin{array}{l}\text { C.E. Beavers \& Son, Barnett \& Giggs \#4 (dry hole) } \\
\text { TE\&L Co. Suv Blk } 2245750 \mathrm{ft} F E L \text { \& } 450 \mathrm{ft} F \mathrm{SL} \text { S } 60 \text { Ac. of SW1/4 of Blk } 2245\end{array}$ & & 700 & 5/14/1936 & $5 / 18 / 1936$ & Sh, blk: 249 \\
\hline 220 & $\begin{array}{l}\text { C.E. Beavers et al., Belknap Coal Co. \#f (dry hole) } \\
\text { TE\&L Co. Suv. Blk } 2240 \text { Acres, } 490 \mathrm{ft} \text { FWL \& } 590 \mathrm{ft} \text { FNL, } 8 \text { mi S fr Olney }\end{array}$ & & 715 & 8/16/1934 & 8/27/1934 & $\begin{array}{l}\text { Sh, blk: } 260 \\
\text { Sh, sdy, blk: } 708\end{array}$ \\
\hline 221 & $\begin{array}{l}\text { C.E. Beavers et al., Belknap Coal Co. \#10 (dry hole) } \\
\text { TE\&L Co. Suv. BIK } 2280 \text { Acres, } 150 \mathrm{ft} \text { each way fr S \& Elines of } 40 \text { Ac. of } \\
\text { S } 80 \text { Ac. Blk } 22 F W L \text { \& } 590 \mathrm{ft} \text { FNL, } 8 \text { mi S fr Olney }\end{array}$ & & 742 & $8 / 3 / 1931$ & 8/27/1931 & $\begin{array}{l}\text { coal: } 150 \\
\text { Sh, blk: } 268 \\
\text { Sh, blk: } 702\end{array}$ \\
\hline 222 & $\begin{array}{l}\text { C.E. Beavers et al., Couper \& Siebold \#2 (dry hole) } \\
\text { TE\&L Co. Suv. Blk } 81,750 \mathrm{ft} \text { FEL \& } 1417 \mathrm{ft} \mathrm{FSL,} \mathrm{S} 100 \text { Ac. Of } 120 \text { Ac. Blk } 81\end{array}$ & & 1212 & $11 / 30 / 1937$ & $12 / 28 / 1937$ & sand; coal: 1169 \\
\hline 223 & $\begin{array}{l}\text { C.E. Beavers et al., Couper \& Siebold \#3 (dry hole) } \\
\text { TE\&L Co. Suv. Blk } 81,450 \mathrm{ft} \text { FEL \& } 1717 \mathrm{ft} \mathrm{FNL}\end{array}$ & & 1196 & $2 / 8 / 1938$ & 3/6/1938 & $\begin{array}{l}\text { Sh, blk: } 755 \\
\text { Sh, blk: } 1148\end{array}$ \\
\hline 224 & $\begin{array}{l}\text { C.E. Beavers et al., N. Finch \#1 (dry hole) } \\
\text { TE\&L Co. Suv. Blk } 33,150 \mathrm{ft} \mathrm{FEL} \mathrm{\&} 450 \mathrm{ft} \text { FNL }\end{array}$ & & 752 & 2/24/1931 & 3/4/1931 & $\begin{array}{l}\text { Sh, blk: } 283 \\
\text { coal: } 370 \\
\text { Sh, blk: } 717\end{array}$ \\
\hline 225 & $\begin{array}{l}\text { C.E. Beavers et al., I. Gathright \#1 (dry hole) } \\
\text { TE\&L Co. Suv. Blk } 1801,450 \mathrm{ft} \text { FNL \& } 750 \mathrm{ft} \text { FEL, } 40 \text { Ac. }\end{array}$ & & 878 & 11/25/1936 & 12/1/1936 & $\begin{array}{l}\text { Sh, blk: } 400 \\
\text { Sh, blk: } 480 \\
\text { Sh, blk: } 579\end{array}$ \\
\hline 226 & $\begin{array}{l}\text { C.E. Beavers et al., Logan \#2 (oil prod) } \\
\text { TE\&L Co. Suv. Blk } 2251,150 \mathrm{ft} \text { FNL \& } 450 \mathrm{ft} \text { FWL of Blk } 2251\end{array}$ & & 573 & 5/30/1934 & 6/9/1934 & $\begin{array}{l}\text { Sh, blk: } 65 \\
\text { Sh, blk: } 262\end{array}$ \\
\hline 227 & $\begin{array}{l}\text { C.E. Beavers, M.T. Montgomery \#1 (dry hle) } \\
\text { TE\&L Suv. Blok } 3401,150 \mathrm{ft} \text { FEL \& } 870 \mathrm{ft} \mathrm{FSL,} 72 \text { Ac. }\end{array}$ & & 763 & 4/26/1932 & $5 / 1 / 1932$ & $\begin{array}{l}\text { Sh, blk: } 315 \\
\text { Sh, blk: } 375 \\
\text { Sh, blk: } 700\end{array}$ \\
\hline 228 & $\begin{array}{l}\text { C.E. Beavers et al., S. J. Rux \#1 (dry hole) } \\
\text { TE\&L Co. Suv. Blk \#278, } 783 \mathrm{ft} \text { FNL \& } 150 \mathrm{ft} \text { FEL N } 45 \text { Ac. Of E } 60 \text { Ac. SE } 80\end{array}$ & & 515 & 11/4/1931 & 11/7//1931 & $\begin{array}{l}\text { coal: } 150 \\
\text { Sh, blk: } 170\end{array}$ \\
\hline 229 & $\begin{array}{l}\text { C.E. Beavers, G.P. Stewart \#1 1dry hole) } \\
\text { TE\&L Suv. Blk 1492, } 450 \text { ft FNL \& } 150 \mathrm{ft} F W L .65 \text { Ac. }\end{array}$ & & 923 & $12 / 18 / 1935$ & $12 / 29 / 1935$ & $\begin{array}{l}\text { Sh, blk: } 225 \\
\text { Sh, blk: } 406 \\
\text { Sh, blk: } 485\end{array}$ \\
\hline 230 & $\begin{array}{l}\text { C.E. Beavers \& E.C. Reed, M.TD. Montgomery \#1A (oil prod) } \\
\text { TE\&L Co. Suv. Blk } 3401,150 \mathrm{ft} \text { each way fr SW Corner, Blk } 3401\end{array}$ & & 709 & 6/2/1933 & $6 / 7 / 1933$ & $\begin{array}{c}\text { Sh, blk: } 148 \\
\text { Sh, blk: } 297 \\
\text { Sh, blk: } 325 \\
\text { Sh, blk: } 353 \\
\text { Sh, blk: } 385 \\
\text { Sh, blk: } 476 \\
\text { Ist; Sh, blk: } 700 \\
\text { Ist; Sh, blk: } 703.5\end{array}$ \\
\hline 231 & $\begin{array}{l}\text { Beavers, White \& Duncan, Remington-Belknap \#1 (oil prod) } \\
\text { TE\&LL Suv. Blk 2248, } 150 \mathrm{ft} \mathrm{FNL} \mathrm{\&} 750 \mathrm{ft} \text { FEL }\end{array}$ & & 572 & 1/18/1934 & 1/29/1934 & $\begin{array}{l}\text { Sh, blk: } 65 \\
\text { Sh, blk: } 230 \\
\text { Sh, blk: } 270\end{array}$ \\
\hline 232 & $\begin{array}{l}\text { Beavers, White \& Duncan, Remington-Belknap \#2 (oil prod) } \\
\text { TE\&L Suv. Blk } 2248,150 \mathrm{ft} \mathrm{FNL} \mathrm{\&} 1050 \mathrm{ft} \text { FEL }\end{array}$ & & 582 & $2 / 14 / 1934$ & $2 / 24 / 1934$ & $\begin{array}{l}\text { Sh, blk: } 238 \\
\text { Sh, blk: } 274 \\
\text { Sh, blk: } 305\end{array}$ \\
\hline 233 & $\begin{array}{l}\text { Beavers, White \& Duncan, Remington-Belknap \#3 (oil prod) } \\
\text { TE\&L Suv. Blk 2248, } 105 \mathrm{ft} \text { FEL \& } 150 \mathrm{ft} \text { FSL }\end{array}$ & & 594 & $3 / 5 / 1934$ & 3/13/1934 & $\begin{array}{l}\text { Sh, blk: } 80 \\
\text { Sh, blk: } 245 \\
\text { Sh, blk: } 270 \\
\text { Sh, blk: } 310\end{array}$ \\
\hline 234 & $\begin{array}{l}\text { Beavers, White \& Duncan, Remington-Belknap } \# \text { (dry hole) } \\
\text { TE\&L Suv. Blk } 2248,150 \mathrm{ft} \mathrm{FEL} \mathrm{\&} 150 \mathrm{ft} \mathrm{FSL} \mathrm{s} 1 / 2\end{array}$ & & 600 & 3/19/1934 & $4 / 6 / 1934$ & $\begin{array}{l}\text { Sh, blk: } 255 \\
\text { Sh, blk: } 283\end{array}$ \\
\hline 235 & $\begin{array}{l}\text { Beavers, White \& Duncan, , Remington-Belknap \#7 (oil prod) } \\
\text { TE\&L Suv Blk } 224815 \mathrm{ft} F \mathrm{SL} \text { \& } 450 \mathrm{ft} \text { FWL }\end{array}$ & & 579 & $7 / 5 / 1934$ & $7 / 11 / 1934$ & $\begin{array}{l}\text { Sh, blk: } 229 \\
\text { Sh, blk: } 235 \\
\text { Sh, blk: } 260 \\
\text { Sh, blk: } 303\end{array}$ \\
\hline 236 & $\begin{array}{l}\text { Beavers, White \& Duncan, Remington-Belknap \#6 (oil prod) } \\
\text { TE\&L Suv Blk } 2248150 \mathrm{ft} \text { FSL \& } 150 \mathrm{ft} \mathrm{FWL}\end{array}$ & & 585 & $6 / 20 / 1934$ & $7 / 4 / 1934$ & $\begin{array}{l}\text { Sh, blk: } 225 \\
\text { Sh, blk: } 240 \\
\text { Sh, blk: } 265 \\
\text { Sh, blk: } 311 \\
\text { Sh, blk: } 322 \\
\text { Sh, blk: } 345\end{array}$ \\
\hline 237 & $\begin{array}{l}\text { Beavers, White \& Duncan, Remington-Belknap \#8 (oil prod) } \\
\text { TE\&LL Suv BIk } 2248150 \mathrm{ft} F N L \text { \& } 150 \mathrm{ft} \text { FEL. N1/2 of Sec. }\end{array}$ & & 608 & $7 / 2 / 1934$ & 7/17/1934 & $\begin{array}{l}\text { Sh, blk: } 125 \\
\text { Sh, blk: } 251\end{array}$ \\
\hline 238 & $\begin{array}{l}\text { A.D.Beck, Garvey \#6 (oil prod) } \\
\text { TE\&L Suv., Blk } 1859,150 \mathrm{ft} \text { FWL \& } 848 \mathrm{ft} \text { FNL }\end{array}$ & 1125.5 & 745 & 11/20/1961 & 11/25/1961 & Sh, blk: 736 \\
\hline 239 & $\begin{array}{l}\text { Belfort Oil Co., V. Holcomb \#B-1 (oil prod) } \\
\text { T\&NOR Co. Suv. Abst. } 1244,330 \mathrm{ft} \text { FWL \& } 450 \mathrm{ft} \text { FSL }\end{array}$ & 1199 & 3847 & $7 / 3 / 1943$ & $7 / 23 / 1943$ & sh; coal: 2500 \\
\hline 240 & $\begin{array}{l}\text { Belknap Coal Co., Belknap Coal Co \#1 (oil prod) } \\
\text { TE\&L Suv Sec. \#22, } 172 \mathrm{ft} N \& 1316 \mathrm{ft} \text { of SWW Cor. }\end{array}$ & 1177 & 705 & 11/1/1930 & 11/8/1930 & Sh, blk: 265 \\
\hline 241 & $\begin{array}{l}\text { Belknap Oil Co., Belknap Coal Co. \#10 (oil prod) } \\
\text { TE\&L Suv. Sec. 22, } 1916 \mathrm{ft} \text { \& } 150 \mathrm{ft} \mathrm{N} f \mathrm{~S} \text { SW Cor. Lse. }\end{array}$ & 1189 & 716 & $10 / 14 / 1931$ & $10 / 25 / 1931$ & $\begin{array}{c}\text { coal: } 118 \\
\text { Sh, blk: } 280\end{array}$ \\
\hline 242 & Calude Bell, C.P. Stewart \#1 (dry hole) & 1113 & 540 & $6 / 6 / 1933$ & $7 / 12 / 1933$ & $\begin{array}{l}\text { Sh, blk: } 40 \\
\text { coal: }: 3 \\
\text { Sh, blk: } 96 \\
\text { Sh, blk: } 490\end{array}$ \\
\hline 243 & $\begin{array}{l}\text { Bennett \& Bennett, R.B. Allison \#1 (dry hole) } \\
\text { TE\&LL Co. Suv. Blk 266, Abs. } 513,750 \mathrm{ft} E \& 1200 \mathrm{ft} \text { of SW Cor. of Suv \#266 }\end{array}$ & & 813 & $8 / 1 / 1945$ & $8 / 3 / 1945$ & $\begin{array}{l}\text { Sh strks coal: } 450 \\
\text { Sh, bl: } 413 \\
\text { Sh, blk: } 422 \\
\text { Sh, sdy; coal strk: } 608 \\
\text { Sh; coal strks: } 660 \\
\text { Sh, blk: } 793\end{array}$ \\
\hline 244 & $\begin{array}{l}\text { Bennett \& Bennett, W.W. Bailey \#1 (dry hole) } \\
\text { TE\&L Co. Suv. Blk } 60,80 \text { Ac, } 450 \mathrm{ft} \mathrm{N} \& 450 \mathrm{ft} \text { W of SE Cor. Of w1/2 of TE\&L } \\
\text { Co. Suv. 60, } 1 / 2 \mathrm{mi} \text { W fr Orth }\end{array}$ & & 870 & $9 / 18 / 1945$ & 9/23/1945 & $\begin{array}{l}\text { Sh, blk; coal: } 373 \\
\text { caal: } 473 \\
\text { Sh; coal strks: } 672 \\
\text { Sh, blk: } 856\end{array}$ \\
\hline 245 & $\begin{array}{l}\text { Bennett \& Bennett, H. Nennig \#1 (dry hole) } \\
\text { TE\&L Co. Suv. Blk } 65,80 \text { Ac., } 1050 \mathrm{ft} \mathrm{FEL} \mathrm{\&} 1716 \mathrm{ft} \text { FNL of Suv. } 65, \mathrm{~S} 80 \text { of } \\
\text { N } 120 \text { ac, E1/2 Suv. \#65, } 7 \mathrm{mi} \text { SW fr Olney }\end{array}$ & & 1105 & $7 / 23 / 1945$ & $7 / 25 / 1945$ & $\begin{array}{l}\text { Sh; coals strks: } 456 \\
\text { Sh; coal strks: } 596\end{array}$ \\
\hline 246 & $\begin{array}{l}\text { Bennett \& Bennett, J.K. Jeffery \#1 (dry hole) } \\
\text { TE\&L Co. Suv., Blk 52, A-328, } 40 \text { Ac., } 575 \mathrm{ft} \text { \& \& } 1250 \mathrm{ft} \text { of NW Cor. Suv \#52, } \\
1,25 \mathrm{mi} \text { SW fr Orth }\end{array}$ & & 879 & $8 / 4 / 1945$ & $8 / 8 / 1945$ & $\begin{array}{l}\text { Sh; coal strks: } 293 \\
\text { S, hd; coal: } 475 \\
\text { Sh; coal: } 530\end{array}$ \\
\hline 247 & $\begin{array}{l}\text { Bennett \& Bennett, H.L. Whitehead \#2 (dry hole) } \\
\text { TE\&L Co. Suv. Blk. } 166,80 \text { Ac., } 450 \mathrm{ft} \text { N \& } 2040 \mathrm{ft} \text { W of SE Cor. Of Suv. 166, } \\
1,75 \text { mi NW fr Orth }\end{array}$ & & 890 & 10/16/945 & $10 / 19 / 1945$ & $\begin{array}{c}\text { coal: } 469 \\
\text { Sh; coal strks: } 816\end{array}$ \\
\hline 248 & $\begin{array}{l}\text { Benson Estate, S.M.J.J. Benson Estate \#1 (dry hole) } \\
\text { TE\&L Co. Suv. Blk 1391, } 40.31 \text { Ac., } 150 \text { ft fr N \& W lines }\end{array}$ & & 965 & $11 / 5 / 1936$ & $11 / 10 / 1936$ & $\begin{array}{l}\text { Sh, blk: } 425 \\
\text { Sh, blk: } 510 \\
\text { Sh, blk: } 862\end{array}$ \\
\hline
\end{tabular}




\begin{tabular}{|c|c|c|c|c|c|c|}
\hline $\begin{array}{l}\text { Occurrence } \\
\text { No. }\end{array}$ & $\begin{array}{l}\text { Well name / } \\
\text { location }\end{array}$ & $\begin{array}{c}\text { Elevation } \\
\quad(\mathrm{ft})\end{array}$ & $\begin{array}{c}\text { Total } \\
\text { depth (ft) }\end{array}$ & $\begin{array}{l}\text { Spud } \\
\text { date }\end{array}$ & $\begin{array}{c}\text { Compl; PB } \\
\text { dates }\end{array}$ & $\begin{array}{l}\text { Coal and black or dark-gray shale } \\
\text { occurrences (depth in ft) }\end{array}$ \\
\hline 249 & $\begin{array}{l}\text { Benson Bros., J.K. Jefferry } \# \text { ( dry hole) } \\
\text { TE\&L Co. Suv BIK } 52.150 \text { ft FS \& W W lines, } 40 \text { Ac. }\end{array}$ & & 918 & 3/14/1930 & $4 / 7 / 1930$ & $\begin{array}{l}\text { Sh, blk: } 412 \\
\text { Sh blk } 489\end{array}$ \\
\hline 250 & $\begin{array}{l}\text { Benson Bros., B.W. King \#1 (dry hole) } \\
\text { TE\&L Co. Suv. Sec. 631, } 160 \text { Ac., } 150 \mathrm{ft} F W L \text { \& } 1320 \mathrm{ft} \text { FSL, } 2 \text { mi SE fr Jean }\end{array}$ & & 554 & 7/26/1947 & 7/30/1947 & coal: 123 \\
\hline 251 & $\begin{array}{l}\text { Benson Bros., G. McCluer \#1 (dry hole) } \\
\text { TE\&L Co. Suv., Sec. 1947, } 265 \text { Ac., } 1366 \mathrm{ft} \text { fr S \& W lines, } 2 \text { mi SW fr Loving }\end{array}$ & & 551 & 7/16/1947 & 7/23/1947 & Sh, blk: 510 \\
\hline 252 & $\begin{array}{l}\text { Benson Bros., G.D. Leberman \& Wife \#1 (dry hole) } \\
\text { TE\&L Suv. } 271,450 \mathrm{ft} \text { \& } 15 \mathrm{ft} \text { S of NW Corner } 74.2 \text { Ac. Tr. }\end{array}$ & & 703 & $5 / 20 / 1930$ & $5 / 29 / 1930$ & $\begin{array}{l}\text { Sh, blk: } 203 \\
\text { Sh, blk: } 337\end{array}$ \\
\hline 253 & $\begin{array}{l}\text { Calvin Benson et al., E.R. Prideaux \#1 (oil prod) } \\
\text { CTRR Co. \#1 Sur., } 300 \mathrm{ft} \text { FNL \& } 155 \mathrm{ft} \text { FEL of Lse., } 120 \mathrm{Ac} .\end{array}$ & & 617 & $5 / 15 / 1955$ & $7 / 8 / 1955$ & $\begin{array}{c}\text { coal: } 117 \\
\text { Sh, blk: } 555 \\
\text { Sh, blk: } 590\end{array}$ \\
\hline 254 & $\begin{array}{l}\text { B.A. Benton, N. Prideaux \#1 (dry hole) } \\
\text { I\&GN Ry Suv., Abst. 1324, } 150 \mathrm{ft} \text { FSL \& } 150 \mathrm{ft} \text { FWL, } 16.5 \mathrm{Ac} .\end{array}$ & $\sim 1059$ & 1/9/1902 & 3/10/1930 & 4/13/1930 & $\begin{array}{l}\text { Sh, blk: } 130 \\
\text { Sh, blk: } 304 \\
\text { Sh, blk: } 670 \\
\end{array}$ \\
\hline 255 & $\begin{array}{l}\text { W.E. Bernhard, L.L. Anderson Est. \#1 (dry hole) } \\
\text { TE\&L Co. Suv. 269, } 35.35 \text { Ac., } 150 \mathrm{ft} \text { FWL \& } 200 \mathrm{ft} \text { FSL }\end{array}$ & & 507 & 10/4/1936 & 10/18/1936 & $\begin{array}{c}\text { coal: } 100 \\
\text { Sh, blkSh, blk: } 193 \\
\text { Lst; Sh, blkSh, blk: } 406\end{array}$ \\
\hline 256 & $\begin{array}{l}\text { Belknap Coal Co., Belknap Coal Co. \#2 (oil prod) } \\
\text { TE\&L Suv., Sec. 22, } 174 \mathrm{ft} N \text { \& } 1016 \mathrm{ft} \text { Efr SW Cor. Lse. }\end{array}$ & 1175.7 & 701.8 & $11 / 11 / 1930$ & 11/22/1930 & $\begin{array}{c}\text { Sh, blk: } 650 \\
\text { Sh, blkSh, blk: } 690\end{array}$ \\
\hline 257 & $\begin{array}{l}\text { Belknap Coal Co., Belknap Coal Co. \#3 (oil prod) } \\
\text { TE\&\&L Suv., Sec. 22, } 159 \mathrm{ft} \text { S \& } 1420 \mathrm{ft} \text { ffr SW Cor. }\end{array}$ & 1174 & 671.5 & $11 / 16 / 1930$ & $11 / 26 / 1930$ & $\begin{array}{l}\text { Sh, blk: } 93 \\
\text { coal: } 327 \\
\end{array}$ \\
\hline 258 & $\begin{array}{l}\text { Belknap Coal Co., Belknap Coal Co. \#4 (oil prod) } \\
\text { TE\&LL Suv., Sec. 22, } 150 \mathrm{ft} S \& 1720 \mathrm{ft} \text { fr NW Cor. of Lse. }\end{array}$ & 1184 & 669 & 12/2/1930 & $12 / 10 / 1930$ & $\begin{array}{c}\text { coal: } 75 \\
\text { coal, brkn: } 287\end{array}$ \\
\hline 259 & $\begin{array}{l}\text { Belknap Coal Co., Belknap Coal Co. \#5 (oil prod) } \\
\text { TE\&L Co. Suv., } 386 \mathrm{ft} N \& 47 \mathrm{ft} \text {. out of SW Cor. of Lse. }\end{array}$ & 1174 & 665 & 12/18/1930 & 12/27/1930 & $\begin{array}{l}\text { Sh, blk: } 268 \\
\text { Sh, blk: } 310 \\
\text { Sh, blk: } 504\end{array}$ \\
\hline 260 & $\begin{array}{l}\text { Belknap Coal Co., Belknap Coal Co. \#6 (dry hole) } \\
\text { TE\&L Co. Suv., Sec. 22, } 150 \text { ft fr N \& W lines }\end{array}$ & 1165 & 706 & $12 / 14 / 1930$ & 12/20/1930 & $\begin{array}{l}\text { coal: } 125 \\
\text { Sh, blk: } 273 \\
\text { coal: } 315\end{array}$ \\
\hline 261 & $\begin{array}{l}\text { Belknap Coal Co., Belknap Coal Co. \#7 (oil prod) } \\
\text { TE\&LL Suv., Sec. 22, } 1316 \mathrm{ft} \text { E \& } 472 \mathrm{ft} \text { N out of SW Corner, } 53.8 \mathrm{Ac} .\end{array}$ & & 707.5 & 9/9/1931 & 9/18/1931 & $\begin{array}{l}\text { Sh, blk: } 100 \\
\text { Sh, llk: } 253 \\
\text { Sh, blk: } 288 \\
\text { coal: } 330\end{array}$ \\
\hline 262 & $\begin{array}{l}\text { Belknap Coal Co., Belknap Coal Co. \#8 (oil prod) } \\
\text { TE\&L Suv., Sec. } 22,1616 \text { ft E \& } 150 \mathrm{ft} N \mathrm{Nf} \text { SW Corner of Lse. }\end{array}$ & 1182 & 701 & 9/24/1931 & 10/4/1931 & $\begin{array}{l}\text { Sh, blk: } 275 \\
\text { coal: } 334 \\
\text { Sh, blk: } 427\end{array}$ \\
\hline 263 & $\begin{array}{l}\text { Belknap Coal Co., Belknap Coal Co. \#9 (oil prod) } \\
\text { TE\&L Suv., Sec. 22, } 150 \mathrm{ft} \text { \& \& } 2027 \mathrm{ftE} \text { ot of NW Corner of Lse. } \\
\text { W.E. Bernhard, Belknap Coal Co. \#1-B (dry hole) } \\
\text { TE\&L Co. \#8, N } 40 \text { Ac of NE 1/4, } 445 \mathrm{ft} \text { FNL \& 907.7 FEL, } 445 \mathrm{ft} \mathrm{FSL}\end{array}$ & 1138 & 709.5 & $4 / 12 / 1931$ & $\begin{array}{l}10 / 18 / 1931 \\
4 / 18 / 1931\end{array}$ & Sh, blk: 322 \\
\hline 264 & $\begin{array}{l}\text { W.E. Bernhard, Belknap Coal Co. \#C-1 (dry hole) } \\
\text { TE\&L Co. \#10, } 466.6 \mathrm{ft} \text { FSL \& } 933.3 \mathrm{ft} \text { FLL }\end{array}$ & 1165.5 & 527.5 & $4 / 22 / 1931$ & $4 / 27 / 1931$ & $\begin{array}{l}\text { Sh, blk: } 82 \\
\text { coal: } 120 \\
\text { Sh, blk: } 434\end{array}$ \\
\hline 265 & $\begin{array}{l}\text { W.E. Bernhard, Belknap Coal Co. \#1-D (dry hole) } \\
\text { TE\&L Co. Suv 11, } 40 \text { Acres, } 150 \mathrm{ft} \text { FNL \& } 660 \mathrm{ft} \text { FWL }\end{array}$ & & 525 & $11 / 28 / 1934$ & 12/8/1934 & $\begin{array}{l}\text { Sh, blk: } 92 \\
\text { coal: } 104 \\
\text { Sh, blk: } 132 \\
\text { Sh, blk: } 442\end{array}$ \\
\hline 266 & $\begin{array}{l}\text { W.E. Bernhard, W.E. Bernhard \#1 (dry hole) } \\
\text { TE\& \& Co. Suv } \# 8,1050 \mathrm{ft} \text { FNL \& } 466.6 \mathrm{ft} \mathrm{fr} \text { \& \& lines }\end{array}$ & 1125 & 700 & 2/25/1931 & $3 / 11 / 1931$ & $\begin{array}{l}\text { coali: } 95 \\
\text { coal: } 115 \\
\text { Sh, blk: } 642\end{array}$ \\
\hline 267 & $\begin{array}{l}\text { W.E. Bernhard, Morrison Estate \#1 (dry hole) } \\
\text { TE\&L Co. Suv Sec. } 646,320 \text { Acres, } 1460 \mathrm{ft} \text { FEL \& } 1930 \mathrm{ft} \mathrm{FSL}\end{array}$ & 1160 & 508 & 1/26/1932 & $2 / 1 / 1932$ & Sh, blk: 168 \\
\hline 268 & $\begin{array}{l}\text { Bernsen \& O'Rourke, M. Harlan \#6 (oil prod) } \\
\text { TE\&L Co. Suv. Blk 1972, Abs. 1046, } 1650 \mathrm{ft} F S L \text { \& } 330 \mathrm{ft} \text { FEL of Suv. \# } 1972\end{array}$ & 1205 & 3066 & 9/10/1946 & 10/29/1946 & $\begin{array}{l}\text { Sh, dark gy: } 1545 \\
\text { Sh, dark gy: } 1690 \\
\text { Sh, dark gy: } 1735 \\
\text { Sh, dark gy: } 1870 \\
\text { Sh, dark gy: } 2000\end{array}$ \\
\hline 269 & $\begin{array}{l}\text { Biggerstaff \& Gordon, L.D. Box \#2 (dry hole) } \\
\text { TE\&LL Suv., } 119.62 \mathrm{Ac}, 300 \mathrm{ft} \mathrm{FEL,} 300 \mathrm{ft} F \mathrm{FEL}, 30 \mathrm{ft} \mathrm{NW} \text { of } \# 1,120 \text { ac., Blk } 1911, \\
2 \mathrm{mi} \text { NE fr Jean }\end{array}$ & & 595 & 8/18/1951 & 9/13/1951 & $\begin{array}{c}\text { coal: } 189 \\
\text { coal: } 250 \\
\text { Sh, blk: } 541\end{array}$ \\
\hline 270 & $\begin{array}{l}\text { P. Bilbrey \& W. Albritton, D. Johnson \#1 (dry hole) } \\
\text { TE\&L Co. Abst \# } 301 \text { Sur Blok 25, } 160 \text { ac., } 800 \mathrm{ft} \text { FWL \& } 1400 \mathrm{ft} \text { FSL of Lse, } \\
2 \mathrm{mi} \text { SW fr Orth }\end{array}$ & & 719 & $7 / 10 / 1953$ & $7 / 25 / 1953$ & $\begin{array}{l}\text { coulial: } 318 \\
\text { coal } 354 \\
\text { coal: } 385\end{array}$ \\
\hline 271 & $\begin{array}{l}\text { P. Bilbrey \& W. Albritton, Shanafelt \#1 (dry hole) } \\
\text { A. Clingburg Abst 1721, } 160 \text { Ac., } 150 \mathrm{ft} f \mathrm{fr} \& \mathrm{E} \text { lines of W/2 of Lse, } \\
3.5 \mathrm{mi} \text { NE fr Loving }\end{array}$ & & 803 & 11/30/1951 & 12/3/1951 & $\begin{array}{l}\text { coal: }: 5 \\
\text { coal: } 592\end{array}$ \\
\hline 272 & $\begin{array}{l}\text { A.B. Bissey et al., Barnett \& Gibbs \#1 (dry hole) } \\
\text { TE\&L Co. Suv., Blk 2245, } 150 \mathrm{ft} \text { FNL \& } 1866 \mathrm{ft} \text { FWL }\end{array}$ & & 770 & $3 / 11 / 1931$ & 3/24/1931 & $\begin{array}{l}\text { Sh, blk: } 275 \\
\text { Sh, blk: } 720\end{array}$ \\
\hline 273 & $\begin{array}{l}\text { A.B. Bissey, J.A. Cunningham \#1 (dry hole) } \\
\text { TE\&LL Suv., Blk 1912, Abst.\#986, } 1100 \text { ft FNL \& } 750 \mathrm{ft} \text { FEL, } 100 \text { acres. }\end{array}$ & & 600 & 9/27/1937 & 10/2/1937 & $\begin{array}{l}\text { coal: } 125 \\
\text { Sh, blk: } 191 \\
\text { Sh, blk: } 502\end{array}$ \\
\hline 274 & $\begin{array}{l}\text { A.B. Bissey et al., Terrel Est. \#1 (dry hole) } \\
\text { TE\&L Co. Suv., Blk 248, Abs. } 495,150 \mathrm{ft} \mathrm{FNL} \mathrm{\&} 1050 \mathrm{ft} \text { FWL of } 50 \text { ac tr. }\end{array}$ & 1155 & 900 & 8/12/1931 & 8/22/1931 & $\begin{array}{l}\text { Sh, blk: } 320 \\
\text { Sh, bl: } 523 \\
\text { Sh, blk: } 698 \\
\text { Sh, blk: } 874\end{array}$ \\
\hline 275 & $\begin{array}{l}\text { Black Gold Petroleum Co., J.M. Kirkland \#1 (dry hole) } \\
\text { TE\&L Co. Blk 2250, } 150 \mathrm{ft} \mathrm{FNL} \mathrm{\&} 450 \mathrm{ft} \mathrm{FEL}\end{array}$ & & 750 & $10 / 25 / 1934$ & $11 / 3 / 1934$ & $\begin{array}{l}\text { Sh, blk: } 40 \\
\text { Sh, blk: } 215 \\
\text { Sh, blk: } 255 \\
\text { Sh, blk: } 325 \\
\text { Sh, blk: } 658\end{array}$ \\
\hline 276 & $\begin{array}{l}\text { M.W. Blair, M.G. Chaney Est. \#1 (dry hole) } \\
\text { W.M. Goode Abs } 1317,160 \text { Ac. } 31 / 4 \text { mi E of Graham }\end{array}$ & & 2209 & 4/8/1960 & $4 / 15 / 1960$ & Sh, blk: 1786 \\
\hline 277 & $\begin{array}{l}\text { M.W. Blair, D. Garrett \#3 (dry hole) } \\
\text { TE\&L Co Sur, Blk 1487, } 132.5 \text { Ac., } 330 \mathrm{ft} \text { fr N\&E lines of Lse, } 4 \text { mi NW fr Loving }\end{array}$ & & 3550 & 6/6/1953 & $6 / 17 / 1953$ & coal: 140 \\
\hline 278 & $\begin{array}{l}\text { M.W. Blair, C. Heard \#1 (dry hole } \\
\text { TE\&L \#305, } 40 \text { Ac., Sec } 552,150 \mathrm{ft} \text { FNL \& } 783 \mathrm{ft} \text { FWL of Lse, } 1.5 \mathrm{mi} \text { E } \\
\text { fr Spring Creek }\end{array}$ & & 1257 & 12/9/1947 & $12 / 11 / 1947$ & $\begin{array}{l}\text { Sh, blk: } 826 \\
\text { Sh, blk: } 1194\end{array}$ \\
\hline 279 & $\begin{array}{l}\text { M.W. Blair, G.H. Roach \#1 (dry hole) } \\
\text { TE\&L Suv., Abst. \#551, Blk } 304,20 \text { Ac., } 150 \mathrm{ft} \mathrm{FEL} \mathrm{\&} 466 \mathrm{ft} \text { FSL of Lse, } \\
1.5 \mathrm{mi} \text { ffr Spring Creek }\end{array}$ & & 1241 & $11 / 30 / 1947$ & $12 / 3 / 1947$ & Sh, blk: 1223 \\
\hline 280 & $\begin{array}{l}\text { M.W. Blair, H. Shanafelt \#1 (dry hole) } \\
\text { BBB\&C \& W.H. Price Surv., Blk 2, Sec 2173, } 320 \text { Acres, } 150 \mathrm{ft} \text { FWL \& } \\
2490 \mathrm{ft} \text { FNL of Lse, } 3 \mathrm{mi} \text { SW fr Loving }\end{array}$ & & 575 & $12 / 3 / 1951$ & $12 / 9 / 1951$ & $\begin{array}{l}\text { coal: } 167 \\
\text { Sh, blk: } 516\end{array}$ \\
\hline 281 & $\begin{array}{l}\text { M.W. Blair, W.B. Todd \#7 (oil prod) } \\
\text { L.E. Hardin Survey Sec. } 1682.97 \text { ac., } 1030 \mathrm{ft} \mathrm{FSL} \mathrm{\&} 1060 \mathrm{ft} \mathrm{FEL}\end{array}$ & & 3565 & 11/28/1956 & $12 / 7 / 1956$ & $\begin{array}{l}\text { S; lignite strks: } 469 \\
\text { lignite; Sh: } 598\end{array}$ \\
\hline 282 & $\begin{array}{l}\text { Bledsee \& Watson, A.C.Watson \#1 (dry hole) } \\
\text { TE\&L Survey, Suv. 250, } 40 \text { Ac., } 150 \text { ft FSL \& } 1150 \mathrm{ft} \text { FEL }\end{array}$ & 1140 & 674 & $6 / 1 / 1934$ & 6/10/1934 & $\begin{array}{c}\text { Sh, blk: } 60 \\
\text { caal: } 190 \\
\text { Sh, blk: } 267\end{array}$ \\
\hline 283 & $\begin{array}{l}\text { Bluebonnet Oil Corp., W.W. Reid \#1 (dry hole) } \\
\text { TE\&L Surv. Sec. } 743,120 \text { acres, } 475 \mathrm{ft} \text { FNL \& } 1263 \mathrm{ft} \text { FWL } 3 \mathrm{mi} \text { S of Proffitt }\end{array}$ & & 3827 & 7/9/1956 & $8 / 31 / 1956$ & S; coal: 290 \\
\hline 284 & $\begin{array}{l}\text { W.F. Bocchoff, Trustee, C.C. Burton \#1 (dry hole) } \\
\text { TE\&L Co Sur, A-460, Sec 213, } 80 \text { Ac., } 750 \mathrm{ft} \text { FEL \& } 1200 \mathrm{ft} \text { FNL of Blk 213, } \\
4 \text { mi SE fr Olney }\end{array}$ & & 940 & 10/22/1952 & 10/23/1952 & coal: 864 \\
\hline 285 & $\begin{array}{l}\text { W.H. Bockhoff et al., E. Jaster \#1 (oil prod) } \\
\text { I. Holeman Suv. Blk } 130,80 \text { Ac., } 150 \mathrm{ft} \text { FSL \& } 450 \mathrm{ft} \text { FWL of Lse }\end{array}$ & & 1231 & 9/16/1947 & 10/14/1947 & $\begin{array}{l}\text { Sh, blk: } 1201 \\
\text { Sh, blk: } 1219\end{array}$ \\
\hline 286 & $\begin{array}{l}\text { W.F. Bockhoff, Trustee, I.G. Roach \#2 (dry hole) } \\
\text { TE\&L Co Sur, Sec } 196,80 \text { ac, } 450 \mathrm{ft} \text { FNL \& } 150 \mathrm{ft} \text { FEL of Lse, } 5 \mathrm{mi} \text { SW Olney }\end{array}$ & & 1079 & 1/28/1953 & 1/29/1953 & Sh, blk: 1011 \\
\hline 287 & $\begin{array}{l}\text { W.F. Bockhoff, Trustee, T.J. Routon \#1 (dry hole) } \\
\text { TE\&L Sur, Blk 181, Sec A-428, } 80 \text { Ac., } 750 \mathrm{ft} \text { FNL \& } 750 \text { ft FWL of Blk 181, } \\
4 \text { mi SW fr Olney }\end{array}$ & & 950 & $-9 / 1 / 1952$ & & Sh, blk: 889 \\
\hline 288 & $\begin{array}{l}\text { Bohner Oil Co., Gilmore \#1 (dry) } \\
\text { YCSL sec } 14 \text { Blk A } 1484,40 \text { ac, } 150 \mathrm{ft} \text { FWL \& } 1050 \text { ft FSLof sec } 14, \\
0.5 \mathrm{mi} \text { W Markley }\end{array}$ & & 590 & 3/7/1961 & 3/10/1961 & $\begin{array}{l}\text { coal: } 93 \\
\text { coal: } 175\end{array}$ \\
\hline 289 & $\begin{array}{l}\text { C.J. Bohner, Logan \#1 (dry hole) } \\
\text { Young Co. Sch. Land Suv., } 15 \mathrm{ft} F \mathrm{WL} \& 1000 \mathrm{ft} \text { FSL, W } 80 \text { Acres }\end{array}$ & & 590 & 10/27/1938 & 11/2/1938 & $\begin{array}{l}\text { Sh, blk: } 105 \\
\text { Sh, blk: } 510\end{array}$ \\
\hline
\end{tabular}




\begin{tabular}{|c|c|c|c|c|c|c|}
\hline $\begin{array}{l}\text { Occurrence } \\
\text { No. }\end{array}$ & $\begin{array}{l}\text { e Well name / } \\
\text { location }\end{array}$ & $\begin{array}{c}\text { Elevation } \\
\quad(\mathrm{ft})\end{array}$ & $\begin{array}{c}\text { Total } \\
\text { depth (ft) }\end{array}$ & $\begin{array}{l}\text { Spud } \\
\text { date }\end{array}$ & $\begin{array}{c}\text { Compl; PB } \\
\text { dates }\end{array}$ & $\begin{array}{l}\text { Coal and black or dark-gray shale } \\
\text { occurrences (depth in } \mathrm{ft} \text { ) }\end{array}$ \\
\hline \multirow[t]{3}{*}{290} & $\begin{array}{l}\text { J.C. Bohner, E.R. Prideaux \#1 (dry hole) } \\
\text { Sulvia Tynes Sur Abs } 274.750 \mathrm{ftFSL} \& 450 \mathrm{ft} \text { FWL } 4 \text { mi NE Loving TX }\end{array}$ & & 648 & $2 / 5 / 1938$ & 2/14/1938 & Sh, blk: 30 \\
\hline & & & & & & $\begin{array}{l}\text { Sh, blk: } 76 \\
\text { coal: } 137\end{array}$ \\
\hline & & & & & & S, blk: 478 \\
\hline \multirow[t]{2}{*}{291} & C.J. Bohner, Predeaux \#1 (dry hole) & & 511 & $3 / 4 / 1941$ & $3 / 10 / 1941$ & coal: 76 \\
\hline & S. Tynes Suv., 40 Acres, 150 ft fr S \& W lines & & & & & Sh, blk: 473 \\
\hline 292 & $\begin{array}{l}\text { C.J. Bohner-B.C. Gilmore, Prideaux Bros. \#1 (dry hole) } \\
\text { W. Stoneman Suv. } 80 \text { Acres, } 1170 \mathrm{~N} \text { of SL \& } 1170 \mathrm{ft} \text { Eof WL. A-250 }\end{array}$ & & 579 & 9/15/1936 & 9/21/1936 & Sh, blk: 78 \\
\hline 293 & $\begin{array}{l}\text { Bohner \& Watson, Prideaux \#1 (oil prod) } \\
\text { Abst. \#68, } 40 \text { Acre Tr. } 150 \mathrm{ft} \text { FNL \& } 150 \mathrm{ft} \mathrm{FEL}\end{array}$ & & 511 & 4/15/1939 & 4/21/1939 & $\begin{array}{l}\text { Sh, blk: } 98 \\
\text { Sh, blk: } 480\end{array}$ \\
\hline \multirow[t]{4}{*}{294} & Bohner \& Watson, Prideaux Bros. \#5 (oil prod) (Markley fld) & & 517 & 8/28/1936 & 9/1/1936 & Sh, blk: 32 \\
\hline & YCSC Suv. Abst 1284, Blk 16, 43 Ac., $300 \mathrm{ft} f \mathrm{fr} \# 3$ \& \#4 & & & & & Sh, blk: 73 \\
\hline & & & & & & Sh, blk: 138 \\
\hline & & & & & & Sh, blk: 475 \\
\hline 295 & $\begin{array}{l}\text { Bohner \& Watson, Prideaux \#10 (oil prod) } \\
\text { YSC Lds Sur. } 123.5 \text { Ac. Tr. } 300 \mathrm{ft} \text { NE of well \#7 }\end{array}$ & & 525 & $5 / 23 / 1940$ & $5 / 26 / 1940$ & Sh, blk: 487 \\
\hline 296 & Bohner \& Watson, Prideaux \#111 (oil prod) & & 535 & $6 / 15 / 1940$ & $6 / 21 / 1940$ & Sh, blk: 497 \\
\hline 297 & $\begin{array}{l}\text { YSC Lds Suv., Blk 16, } 150 \mathrm{ftFEL} 300 \mathrm{ft} \text { of well \#6 } \\
\text { Bohner \& Watson, Prideaux \#12 (oil prod)(Markley fld) }\end{array}$ & & 575 & 9/23/1940 & $10 / 2 / 1940$ & coal: 123 \\
\hline & YSC Lds Suv., Blk 16, 123.5 Ac., 264 ft FEL \& approx. $250 \mathrm{ft} \mathrm{FNL}$ & & & & & Sh, blk: 517 \\
\hline 298 & $\begin{array}{l}\text { Bohner \& Watson, Prideaux \#13 (used as "key well")(Markley Young Co fld) } \\
\text { YSC Lds Suv., } 300 \mathrm{ft} F E L, 300 \mathrm{ft} N \text { of } \# 12\end{array}$ & & 597 & 10/8/1940 & $10 / 14 / 1940$ & coal: 131 \\
\hline 299 & $\begin{array}{l}\text { Bohner \& Watson, Prideaux \#14(Young County -Markley-fld) } \\
\text { YSC Lds Suv., Blk 16, 300 ft FEL, } 300 \mathrm{ft} \text { SW of well \#12 }\end{array}$ & & 579 & $10 / 20 / 1940$ & $11 / 2 / 1940$ & coal: 119 \\
\hline 300 & $\begin{array}{l}\text { C.J. Bohner Oil Corp., Predieaux \#4-A (dry hole) (Young County fld) } \\
\text { S. Tynes Suvv., } 150 \mathrm{ft} \text { FSL, } 300 \mathrm{ft} \text { E of \#3-A }\end{array}$ & & 497 & $11 / 10 / 1940$ & $11 / 20 / 1940$ & coal: 58 \\
\hline 301 & $\begin{array}{l}\text { Bohner \& Watson, Prideaux \#15 (dry hole)/Young County-Markley-fld) } \\
\text { Young County School Ld. Survey, Blk 16, Abt. 1284, } 150 \mathrm{ft} \text { fr S \& Elines }\end{array}$ & & 594 & $5 / 18 / 1941$ & $5 / 26 / 1941$ & coal: 119 \\
\hline 302 & $\begin{array}{l}\text { Bonell Oil co., J.W. Jones \#1 (oil prod) } \\
\text { Susan Sallie Suv. Abst. } 252,40 \text { Acre Tr., } 150 \text { ft FSL \& } 450 \text { ft FWL, } \\
4.5 \mathrm{mi} \text { SW fr Markley. }\end{array}$ & & 518 & $11 / 12 / 1934$ & $12 / 3 / 1934$ & $\begin{array}{l}\text { coal: } 110 \\
\text { Sh, blk: } 509 \\
\text { Sh, blk: } 512\end{array}$ \\
\hline 303 & $\begin{array}{l}\text { Bonell Dil co., R.E. Jones \#1 (dry hole) } \\
\text { S.Sallie Suv. A-252, } 150 \mathrm{ft} F \mathrm{FL} \& 450 \mathrm{ft} \text { FWL NW 1/4 } 40 \text { Acres }\end{array}$ & 1172.3 & 565 & $12 / 16 / 1934$ & $12 / 23 / 1934$ & $\begin{array}{l}\text { coal: } 117 \\
\text { Sh, blk: } 509\end{array}$ \\
\hline 304 & $\begin{array}{l}\text { The Bonner Company, J.W. Davis \#4 (dry hole) } \\
\text { TE\&L Co. Suv. \#1395 }\end{array}$ & & 900 & $10 / 3 / 1929$ & 10/22/1929 & $\begin{array}{l}\text { Sh, blk: } 395 \\
\text { Sh, blk: } 449 \\
\text { Sh, blk: } 463\end{array}$ \\
\hline 305 & The Bonner Company, J.W. Davis \#5 (dry hole) & & 930 & 10/26/1929 & 12/7/1929 & Sh, blk: 421 \\
\hline & TE\&L Co. Suv., $637 \mathrm{ft} \mathrm{FSL,} 300 \mathrm{ft}$ E\#1 & & & & & Sh, blk: 426 \\
\hline & & & & & & Sh, blk: 510 \\
\hline & & & & & & Sh, blk: 837 \\
\hline & & & & & & coal: 910 \\
\hline 306 & The Bonner Company, J.W. Davis \#6 (oil prod) & 1193 & 908 & 12/28/1929 & $3 / 3 / 1930$ & Sh, blk: 424 \\
\hline & TE\&L Company Suv., $800 \mathrm{ft} f \mathrm{fr}$ ? \& $150 \mathrm{ft} \mathrm{FSL}$ & & & & & $\begin{array}{l}\text { Sh, blk: } 501 \\
\text { Sh, blk: } 830\end{array}$ \\
\hline 307 & $\begin{array}{l}\text { The Bonner Company, J.W. Davis \#9 (oil prod) } \\
\text { TE\& L Co. Suv } 300 \mathrm{ft} N \text { NE of } \# 3\end{array}$ & & 896 & $5 / 2 / 1930$ & $5 / 26 / 1930$ & $\begin{array}{l}\text { Sh, blk: } 425 \\
\text { Sh, blk: } 822\end{array}$ \\
\hline 308 & Boyd \& Kimberlin, A.C. McDonald Est. \#3 (dry hole) (Prideaux fld) & & 620 & $8 / 8 / 1940$ & 8/26/1940 & coal: 1111 \\
\hline & CTRR Suv., Abst. 68,80 Ac., $300 \mathrm{ft} \mathrm{FEL} \mathrm{\&} 750 \mathrm{ft}$ FSL, $2.5 \mathrm{mi}$ W fr Markley & & & & & Sh, blk: 547 \\
\hline & & & & & & Sh, blk: 573 \\
\hline 309 & Boyton-Butler-Calhoun, S. J. Benson Estate \#1 (dry hole) & & 1002 & $11 / 29 / 1936$ & $12 / 10 / 1936$ & Sh, blk: 542 \\
\hline & TE\&L Suv., BIk $1398,40 \mathrm{ft} W$ of $\mathrm{L}$ line \& $150 \mathrm{ft} \mathrm{N}$ of $S$ line & & & & & Sh, blk: 560 \\
\hline & & & & & & $\begin{array}{l}\text { Sh, blk: } 901 \\
\text { Sh, blk:260 }\end{array}$ \\
\hline 310 & E.P. Bowen, F. Clarke \#1 (dry hole) & 1180 & 950 & $3 / 19 / 1932$ & 4/2/1932 & $\begin{array}{l}\text { Sh, blk: } 260 \\
\text { Shs bly: } 428\end{array}$ \\
\hline & TE\&L Suv., Sec. 212, $150 \mathrm{ft}$ FNL \& $450 \mathrm{ft} F W L, W 100$ of N $147 \mathrm{Ac}$. & & & & & Sh, blk: 428 \\
\hline & & & & & & Sh, blk: 869 \\
\hline 311 & M.M. Bradley, L.F. Gragg \#1 (dry hole) (Prideaux District) & & 808 & 2/14/1940 & 2/25/1940 & Sh, blk: 95 \\
\hline & A.Rohys Suv. Blk 240, 150 Ac., $975 \mathrm{ft}$ FNL \& $1251 \mathrm{ft} \mathrm{FWL,} 1.5 \mathrm{mi}$ NE fr Farmer & & & & & Sh, blk: 250 \\
\hline & & & & & & Sh, blk: 266 \\
\hline & & & & & & Sh, blk: 334 \\
\hline & & & & & & Sh, blk: 705 \\
\hline 312 & E. Bradshaw, A.C. Spper \#1 (dry hole) & & 558 & & & Sh, blk: 67 \\
\hline & Wm. Stoneham Suv., Abst. \#250, 40 Acres, $150 \mathrm{ft} \mathrm{FSL} \mathrm{\&} 150 \mathrm{ft} \mathrm{FWL}$ & & & & & Sh, blk: 526 \\
\hline 313 & The L.G. Bradstreet Co., Matthews \#1-B (dry hole) & & 724 & 6/19/1929 & 6/25/1929 & Sh, blk: 280 \\
\hline & A. Rihys Survey, $450 \mathrm{ft}$ WL \& $150 \mathrm{ft} \mathrm{SL}$ & & & & & Sh?, blk: 699 \\
\hline 314 & The L.G. Bradstreet Co., L.T. Richardson \#1-A (oil prod) & 1175 & $694 ?$ & $5 / 2 / 1929$ & 5/10/1929 & Sh, blk: 220 \\
\hline & J.G. Maupin Survey & & & & & Sh, blk: 250 \\
\hline & & & & & & coal: 258 \\
\hline & & & & & & Sh, blk: 478 \\
\hline & & & & & & Sh, blk: 681 \\
\hline 315 & The L.G. Bradstreet Co., L.T. Richardson \#2-A (dry hole) & 1154 & 790 & $7 / 30 / 1929$ & 8/14/1929 & Sh, blk: 275 \\
\hline & J.G. Maupin Survey & & & & & Sh, blk: 360 \\
\hline & & & & & & Sh, blk: 687 \\
\hline & & & & & & Sh, blk: 767 \\
\hline 316 & $\begin{array}{l}\text { The L.G. Bradstreet Co., L.T. Richardson \#2-B (dry hole) } \\
\text { J.G. Maupin Survey }\end{array}$ & & 730 & $6 / 9 / 1929$ & 6/16/1929 & Sh, blk: 330 \\
\hline 317 & The L.G. Bradstreet Co., L.T. Richardson \#3-B (dry hole) & & 805 & $7 / 7 / 1929$ & 7/16/1929 & Sh, blk: 258 \\
\hline & J.G. Maupin Survey, $150 \mathrm{ft} \mathrm{NL} \mathrm{\&} 150 \mathrm{ft}$ WL & & & & & Sh, blk: 270 \\
\hline & & & & & & Sh, blk: 344 \\
\hline & & & & & & Sh, blk: 697 \\
\hline 318 & The L.G. Bradstreet Co., L.A. Snider \#1 (oil prod) & 1014 & 564.50 & 1/12/1929 & 1/20/1929 & Sh, blk: 116 \\
\hline & R. Wilson Survey, $510 \mathrm{ft} F W L \& 450 \mathrm{ft} \mathrm{FNL}$ & & & & & Sh, blk: 526 \\
\hline & The L.G. Bradstreet Co., L.A. Snider Estate \#2 (oil prod) & 1021 & 583 & 1/24/1929 & 1/31/1929 & Sh, blk: 536 \\
\hline & R. Wilson Survey, $510 \mathrm{ft} \mathrm{FWL} \mathrm{\&} 750 \mathrm{ft} F \mathrm{NL}$ & & & & & coal: 572 \\
\hline 319 & The L.G. Bradstreet Co., L.A. Snider "A" Lease \#4 (oil prod) & 1015 & 574 & 2/26/1929 & $3 / 4 / 1929$ & Sh, blk: 126 \\
\hline & R. Wilson Survey, $510 \mathrm{ft} \mathrm{FNL} \mathrm{\&} 150 \mathrm{ft}$ FWL & & & & & coal: 191 \\
\hline & & & & & & Sh, blk: 515 \\
\hline 320 & $\begin{array}{l}\text { The L.G. Bradstreet Co., L.A. Snider Estate 所 (oil prod) } \\
\text { R. Wilson Survey, } 150 \mathrm{ft} \text { FEL \& } 150 \mathrm{ft} \text { FNL. }\end{array}$ & 1013 & 566 & $3 / 2 / 1929$ & $3 / 9 / 1929$ & Sh, blk: 518 \\
\hline 321 & C.H. Bradenburg, F.M. Cullern $\# 8$ (dry hole) & & 580 & 4/23/1935 & 4/27/1935 & Sh, blk: 528 \\
\hline & G.N. Stinnett Suv., $300 \mathrm{ft} \mathrm{S}$ of $\# 4 \& 250 \mathrm{ft}$ West. 40 Acre Tract, Abst 2177 & & & & & Sh, blk: 560 \\
\hline 322 & E.S. Braxhear, S.F. Jeffrey \#1 (oil prod) (WC) & 1255 & 863 & 1/25/1950 & 2/22/1950 & Sh; coal strks: 472 \\
\hline & TE\&L Co. Sur. Blk 51, $450 \mathrm{ft} \mathrm{FNL} \mathrm{\&} 150 \mathrm{ft} \mathrm{FWL} \mathrm{of} \mathrm{Lse.,} 79.5$ acres & & & & & Sh, blk: 489 \\
\hline & & & & & & Sh, blk: 519 \\
\hline & & & & & & Sh, blk: 641 \\
\hline & & & & & & Sh, blk: 699 \\
\hline
\end{tabular}




\begin{tabular}{|c|c|c|c|c|c|c|}
\hline $\begin{array}{l}\text { Occurrence } \\
\text { No. }\end{array}$ & $\begin{array}{l}\text { e Well name / } \\
\text { location }\end{array}$ & $\begin{array}{c}\text { Elevation } \\
\quad(\mathrm{ft})\end{array}$ & $\begin{array}{c}\text { Total } \\
\text { depth (ft) }\end{array}$ & $\begin{array}{l}\text { Spud } \\
\text { date }\end{array}$ & $\begin{array}{c}\text { Compl; PB } \\
\text { dates }\end{array}$ & $\begin{array}{l}\text { Coal and black or dark-gray shale } \\
\text { occurrences (depth in ft) }\end{array}$ \\
\hline 323 & $\begin{array}{l}\text { E.S. Brashear, S.R. Jeffery \#C-2 (dry hole) (WC) } \\
\text { TE\&L Co., Blk 51, } 160 \text { Acres, } 600 \mathrm{ft} \text { FSL \& } 450 \mathrm{ft} \text { FEL of Lse, } 0.75 \mathrm{mi} \text { fr Orth }\end{array}$ & & 860 & 1/30/1954 & $2 / 1 / 1954$ & $\begin{array}{c}\text { coal: } 390 \\
\text { Sh; coal: } 415 \\
\text { coal; Sh, sdy: } 525\end{array}$ \\
\hline 324 & $\begin{array}{l}\text { E.S. Brashear, D. Johnson \#1 (dry hole) (WC) } \\
\text { TE\&L Suv. Blk 25, } 23 \text { Ac., } 150 \mathrm{ft} \text { fr } \mathrm{N} \text { \& E lines, } 2 \mathrm{mi} \text { f fr Orth }\end{array}$ & & 785 & 12/8/1940 & 1/16/1941 & $\begin{array}{l}\text { Sh, blk: } 175 \\
\text { Sh, blk: } 200 \\
\text { Sh, blk: } 347 \\
\text { Sh, blk: } 407 \\
\text { Sh, blk: } 778 \\
\text { Sh, blk: } 724 \\
\text { Sh, blk: } 785 \\
\end{array}$ \\
\hline 325 & $\begin{array}{l}\text { E.S. Brashear et al., D. Johnson \#1 (dry hole) (WC) } \\
\text { TE\&L Co. Abst } 301 \text { Surv, Blk } 25,160 \text { ac., } 150 \mathrm{ft} \mathrm{FEL} \mathrm{\&} 1850 \mathrm{ft} \text { FSL }\end{array}$ & & 757 & $8 / 1 / 1953$ & $8 / 4 / 1953$ & $\begin{array}{l}\text { coal: } 380 \\
\text { coal: } 423 \\
\end{array}$ \\
\hline 326 & $\begin{array}{l}\text { P.C. Brashear, A.C. Casey \#1 (dry hole) (NWW Loving fld) } \\
\text { TE\&\&L Co. Suv., Sec } 1905,40 \text { acres, } 150 \mathrm{ft} \text { FEL \& } 300 \mathrm{ft} \mathrm{FSL}\end{array}$ & & 640 & $12 / 12 / 1935$ & $12 / 17 / 1935$ & $\begin{array}{l}\text { Sh, blk: } 300 \\
\text { Sh, blk: } 352 \\
\text { Sh, blk: } 602\end{array}$ \\
\hline 327 & $\begin{array}{l}\text { P.C. Brashear, S.R. Jeffery \#1 (dry hole) (WC) } \\
\text { TE\&L Co. Suv., Blk 50, } 150 \mathrm{ft} \text { FEL \& } 2800 \mathrm{ft} \text { FNL of } 50 \text { Ac Tract. 2mi SW fr Orth }\end{array}$ & 1200 & 800 & $5 / 15 / 1940$ & $5 / 28 / 1940$ & $\begin{array}{l}\text { Sh, blk: } 235 \\
\text { Sh, blk: } 367 \\
\text { Sh, blk: } 435\end{array}$ \\
\hline 328 & $\begin{array}{l}\text { E.S. Brashear et al., S.R. Jeffery } \# 2 \text { (dry hole) (WC) } \\
\text { TE\&L Co. Suv., Blk } 51,80 \text { acres, } 300 \mathrm{ft} \text { FNL \& } 450 \mathrm{ft} \mathrm{FWL} \mathrm{of} \mathrm{NE/4,} \\
0.5 \mathrm{mi} \text { f fr Orth. }\end{array}$ & 1255 & 920 & $2 / 26 / 1950$ & $3 / 7 / 1940$ & $\begin{array}{l}\text { coal; Sh, blk: } 525 \\
\text { Sh, blk: } 855\end{array}$ \\
\hline 329 & $\begin{array}{l}\text { P.C. Brashear, Jefferey } \# 1 \text { (dry hole) (WC) } \\
\text { TE\&L Co. Suv., Blk } 50,30 \text { Acres, } 150 \mathrm{ft} \text { fr N \& E lines, } 2 \mathrm{mi} \mathrm{S} \text { fr Orth }\end{array}$ & 1180 & 826 & $3 / 4 / 1940$ & $3 / 13 / 1940$ & $\begin{array}{l}\text { Sh, blk: } 375 \\
\text { Sh, blk: } 414\end{array}$ \\
\hline 330 & $\begin{array}{l}\text { P.C. Brashear et al., Finch \#1 (dry hole) (Young fld) } \\
\text { TE\&L Co. Suv. \#38, Blk A-314, } 80 \text { acres, } 300 \mathrm{ft} \mathrm{FSL} \mathrm{\&} 850 \mathrm{ft} \text { FWL of NE/4, } \\
4 \mathrm{mi} \text { SW fr Orth }\end{array}$ & 1200 & 745 & $10 / 21 / 1949$ & $10 / 26 / 1949$ & $\begin{array}{l}\text { lignite: } 403 \\
\text { lignite: } 426\end{array}$ \\
\hline 331 & $\begin{array}{l}\text { P.C. Brashear, Finch \#2 (dry hole) (Young fld) } \\
\text { TE\&L Co. Suv. \#38, Blk A-314, } 80 \text { acres, } 150 \mathrm{ft} \text { FSL \& } 850 \mathrm{ft} \text { FWL of NE/ } 4 \text { of } \\
\text { Blk } 38,4 \mathrm{mi} \text { SW fr Orth }\end{array}$ & 1195 & 738.5 & $11 / 10 / 1949$ & 12/7/1949 & $\begin{array}{l}\text { Sh, blk: } 185 \\
\text { Sh, blk: } 338 \\
\text { Sh, blk: } 435 \\
\end{array}$ \\
\hline 332 & $\begin{array}{l}\text { P.C. Brashear et al., T.J. Routon \#1 (dry hole) (WC) } \\
\text { TE\&L Co. Sur, Blk } 24,311 \text { Ac., } 1325 \mathrm{ft} \text { FSL \& } 1510 \mathrm{ft} \text { FEL of Lse., A-300, } \\
3 \mathrm{mi} \text { S fr Orth }\end{array}$ & & 725 & $8 / 11 / 1953$ & $8 / 12 / 1953$ & $\begin{array}{c}\text { coal; Sh: } 265 \\
\text { Sh; coal strks: } 358 \\
\text { Sh, blk: } 649 \\
\text { coal: } 681\end{array}$ \\
\hline 333 & $\begin{array}{l}\text { P.C. Brashear et al., T.J. Routon \#2 (dry hole) (WC) } \\
\text { TE\&L Co. Sur, Blk 24, } 311 \text { Ac., } 1510 \mathrm{ft} \text { FEL \& } 1625 \mathrm{ft} F S L \text { of Lse., A-300, } \\
3 \mathrm{mi} \text { S fr Orth }\end{array}$ & & 750 & 8/20/1953 & 8/21/1953 & $\begin{array}{c}\text { coal: } 158 \\
\text { coal: } 325 \\
\text { Sh, blk: } 668\end{array}$ \\
\hline 334 & $\begin{array}{l}\text { Brashear \& Wheeler, JK. Jeffery \#1-A (oil prod) } \\
\text { TE\&LL Sur, Sec. \#42, } 15 \text { acres, } 150 \mathrm{ft} \text { fr N \& E lines in SW/4 }\end{array}$ & 1167.5 & 731 & $4 / 4 / 1931$ & $4 / 13 / 1931$ & $\begin{array}{l}\text { Sh, blk: } 345 \\
\text { Sh, blk: } 415\end{array}$ \\
\hline 335 & $\begin{array}{l}\text { O. Bratcher et al., } . \text {... Finch \#1 (dry hole) } \\
\text { TE\&\&L Co. Suvv., Sec } 32,40 \text { Acres, } 450 \text { ft FNL \& } 150 \text { ft FEL. }\end{array}$ & 1140.5 & 718 & $6 / 11 / 1931$ & 6/24/1931 & $\begin{array}{l}\text { coal: } 112 \\
\text { Sh, blk: } 142 \\
\text { Sh, blk: } 267 \\
\text { Sh, blk: } 273 \\
\text { Sh, blk: } 305\end{array}$ \\
\hline 336 & $\begin{array}{l}\text { 0. Bratcher, N.R. Finch \#1 (dry hole) } \\
\text { TE\&L Co. Suv., Sec } 38,150 \mathrm{ft} \text { FWL \& } 750 \mathrm{ft} \mathrm{FNL,} \mathrm{W/2} \mathrm{of} \mathrm{NW/4} \mathrm{of} \mathrm{Sec} 38, \text { A } 314 \\
\text { (Gunsight lime @ 639 ft) }\end{array}$ & & 850 & $8 / 7 / 1931$ & \begin{tabular}{ll|}
$8 / 16 / 1931$ \\
\end{tabular} & $\begin{array}{l}\text { Sh, blk: } 250 \\
\text { Sh, dark: } 400 \\
\text { Sh, blk: } 435 \\
\text { Sh, blk: } 765\end{array}$ \\
\hline 337 & $\begin{array}{l}\text { Brather \& Walton, E.R. Prideaux \#1 (oil prod) } \\
\text { Sylphia Tynes Survey, } 150 \mathrm{ft} \text { FNL \& } 150 \mathrm{ft} F \mathrm{FWL}\end{array}$ & 1143 & 462 & $2 / 14 / 1929$ & $2 / 18 / 1929$ & $\begin{array}{l}\text { coal: } 60 \\
\text { coal: } 125 \\
\text { Sh, blk: } 436\end{array}$ \\
\hline 338 & $\begin{array}{l}\text { Brather \& Walton, E.R. Prideaux \#2 (oil prod) } \\
\text { Sylphia Tynes Survey, } 300 \mathrm{ft} \text { Eof \#1 }\end{array}$ & 1148 & 485 & $2 / 22 / 1929$ & $2 / 26 / 1929$ & $\begin{array}{c}\text { coal: } 65 \\
\text { coal: } 130 \\
\text { coal: } 237 \\
\text { Sh, blk: } 445\end{array}$ \\
\hline 339 & $\begin{array}{l}\text { Brazelton Brothers, J.R. Barnett \#1 (dry hole) (Olney field) } \\
\text { TE\&LL Suv., Blk 187, } 60 \text { Ac., } 150 \mathrm{ft} \mathrm{FEL} \mathrm{\&} 450 \mathrm{ft} \text { FSL }\end{array}$ & & 1114 & $\begin{array}{l}/ 26 / 1938 \\
\end{array}$ & 10/5/1938 & $\begin{array}{l}\text { Sh, blk: } 463 \\
\text { Sh, blk: } 495 \\
\text { Sh, blk: } 638 \\
\text { Sh, blk: } 707 \\
\text { Sh, blk: } 1080\end{array}$ \\
\hline 340 & $\begin{array}{l}\text { Brazelton Bros, B.B. Colley \#1 (dry hole) (Olney field) } \\
\text { TE\&L Co. Suv., Abst. 489, Blk 242, } 783 \mathrm{ft} \mathrm{fr} N \text { \& W lines of SE/4 }\end{array}$ & & 884 & 1/19/1937 & 1/24/1937 & $\begin{array}{l}\text { Sh, blk: } 330 \\
\text { Sh, blk: } 345 \\
\text { Sh, blk: } 798\end{array}$ \\
\hline 341 & $\begin{array}{l}\text { Brazelton Brothers \& C.P. Shelton, Barnett \& Gibbs \#B-1 (dry hole) } \\
\text { TE\&L Co. Suv., Blk 2245, } 80 \text { Ac., } 1050 \mathrm{ft} \text { FNL \& } 1800 \mathrm{ft} \text { FWL }\end{array}$ & & 730 & 3/13/1934 & 3/20/1934 & $\begin{array}{l}\text { Sh, blk: } 105 \\
\text { Sh, blk: } 140 \\
\text { Sh, blk: } 250 \\
\text { Sh, blk: } 268\end{array}$ \\
\hline 342 & $\begin{array}{l}\text { Brazelton Brothers, Belknap Coal \& Oil Co. \#4 (dry hole) } \\
\text { TE\&L Suv., Blk 21, } 40 \text { Acres, } 320 \mathrm{ft} \text { SW of Well \#3 }\end{array}$ & & 751 & 11/11/1932 & 11/14/1932 & $\begin{array}{l}\text { Sh, blk: } 105 \\
\text { Sh, blk: } 150 \\
\text { blk: } 275 \\
\text { blk: } 295 \\
\text { Sh. blk: } 307\end{array}$ \\
\hline 343 & $\begin{array}{l}\text { Brazelton Brothers, W.B. Howard \#1 (dry hole) (Olney field) } \\
\text { TE\&L Suv. \#3401, Abst. 1221, } 80 \text { acre tract, } 150 \mathrm{ft} \text { FEL \& } 1214 \mathrm{ft} \mathrm{FNL}\end{array}$ & & 1008 & 3/10/1936 & 3/17/1936 & $\begin{array}{l}\text { Sh, blk: } 468 \\
\text { Sh, blk: } 541 \\
\text { Sh, blk: } 899\end{array}$ \\
\hline 344 & $\begin{array}{l}\text { Brazelton Brothers, McBrayer Estate \#1 (oil prod) } \\
\text { TE\&LL Suv.,\#1904, } 33 \text { Ac. Tr., } 150 \mathrm{ft} \text { fr S \& W lines }\end{array}$ & & 696 & $6 / 19 / 1938$ & 7/3/1938 & $\begin{array}{l}\text { Sh, blk: } 280 \\
\text { Sh, blk: } 355 \\
\text { Sh, blk: } 659 \\
\end{array}$ \\
\hline 345 & $\begin{array}{l}\text { Brazelton Brothers, McBrayer Estate \#2 (dry hole) (Tatum pool, Loving field) } \\
\text { TE\&LL Suv, \#1904, } 33 \text { Ac. Tr., Abst. } 978,150 \mathrm{ft} \text { FSL, } 300 \mathrm{ft} \text { Eof \#1 } \\
3 \mathrm{mi} \text { NE of Jean }\end{array}$ & & 693 & 7/25/1938 & $7 / 30 / 1938$ & $\begin{array}{l}\text { Sh, blk: } 286 \\
\text { Sh, blk: } 350 \\
\text { Sh, blk: } 665\end{array}$ \\
\hline 346 & $\begin{array}{l}\text { Brazelton Brothers, McBrayer Estate \#3 (oil prod) } \\
\text { TE\&L Suv. 1907, } 150 \mathrm{ft} \text { fr N \& WL. } \\
\text { Brazelton Bros, McBrayer Estate \#4 (dry hole) (N.Tx Olney area, Tatum pool) } \\
\text { TE\&L Suv. \#1907, } 58 \text { Acre Tr., } 450 \mathrm{ft} \text { FWL \& } 310 \mathrm{ft} \text { FNL, } 300 \mathrm{ft} \text { Eof \#3. }\end{array}$ & & $\begin{array}{c}11 / 8 / 1901 \\
693\end{array}$ & $\begin{array}{l}/ 24 / 1938 \\
1 / 25 / 1939\end{array}$ & $\begin{array}{l}/ 29 / 1938 \\
1 / 30 / 1939\end{array}$ & $\begin{array}{l}\text { Sh, blk: } 286 \\
\text { Sh, bl: } 350 \\
\text { Sh, blk: } 275 \\
\text { Sh, blk: } 335 \\
\text { Sh, blk: } 665\end{array}$ \\
\hline 347 & $\begin{array}{l}\text { Brazelton Brothers et al, C.U. Taack \#8 (dry hole) } \\
\text { TE\&L Co. Suv. Sec. } 263,60 \text { Acres, } 940 \mathrm{ft} \text { FEL \& } 450 \mathrm{ft} F \mathrm{SL}\end{array}$ & & 756 & $8 / 18 / 1933$ & $824 / 1933$ & $\begin{array}{l}\text { Sh, blk: } 118 \\
\text { coal: } 147 \\
\text { Sh, blk: } 275 \\
\text { Sh, blk: } 340\end{array}$ \\
\hline 348 & $\begin{array}{l}\text { Brazelton Brothers et al, C.J. Taack \#9 (dry hole) } \\
\text { TE\&LL Suv. Sec. 263, Abst. } 510,60 \text { Acres, } 640 \mathrm{ft} \text { FEL \& } 450 \mathrm{ft} \text { FSL } \\
\text { (lime G.S. at } 645 \mathrm{ft} \text { ) }\end{array}$ & & 740 & 8/25/1933 & 9/1/1933 & $\begin{array}{l}\text { Sh, blk: } 136 \\
\text { Sh, blk: } 280 \\
\text { Sh, blk: } 345\end{array}$ \\
\hline 349 & $\begin{array}{l}\text { Brazelton Brothers et al, C.J. Taack \#10 (dry hole) } \\
\text { TE\&L Suv. Sec. 263, Abst. } 510,60 \text { Acres, } 790 \mathrm{ft} \text { FEL \& } 750 \mathrm{ft} \text { FSL }\end{array}$ & & 674 & 9/18/1933 & 9/24/1933 & $\begin{array}{l}\text { (Sh?) blk: } 263 \\
\text { Sh, blk: } 285 \\
\text { Sh, blk: } 342\end{array}$ \\
\hline 350 & $\begin{array}{l}\text { Brazelton Brothers et al, C.J. Taack \#11 (dry hole) } \\
\text { TE\&L Suv. Sec. } 263, \text { Abst. 510, } 60 \text { Acres, } 750 \mathrm{ft} \mathrm{FSL} \mathrm{\&} 1083 \mathrm{ft} \text { FEL } \\
\text { (lime G.S. (SLM) at } 658 \mathrm{ft} \text { ) }\end{array}$ & & 760 & $10 / 12 / 1933$ & $10 / 17 / 1933$ & $\begin{array}{l}\text { (Sh?) blk: } 112 \\
\text { (Sh?) blk: } 130 \\
\text { (Sh?) blk: } 145 \\
\text { (Sh?) blk: } 290 \\
\text { coal: } 343\end{array}$ \\
\hline 351 & $\begin{array}{l}\text { Brazelton Brothers et al, C.J. Taack \#13 (dry hole) } \\
\text { TE\&L Co. Suv. Abst. 510, Blk 263, } 60 \text { Acres, } 940 \mathrm{ft} \mathrm{FEL} \mathrm{\&} 250 \mathrm{ft} F \mathrm{FL}\end{array}$ & & 685 & $4 / 7 / 1934$ & 4/8/1934 & $\begin{array}{l}\text { Sh, blk: } 287 \\
\text { (Sh?) blk: } 344\end{array}$ \\
\hline
\end{tabular}




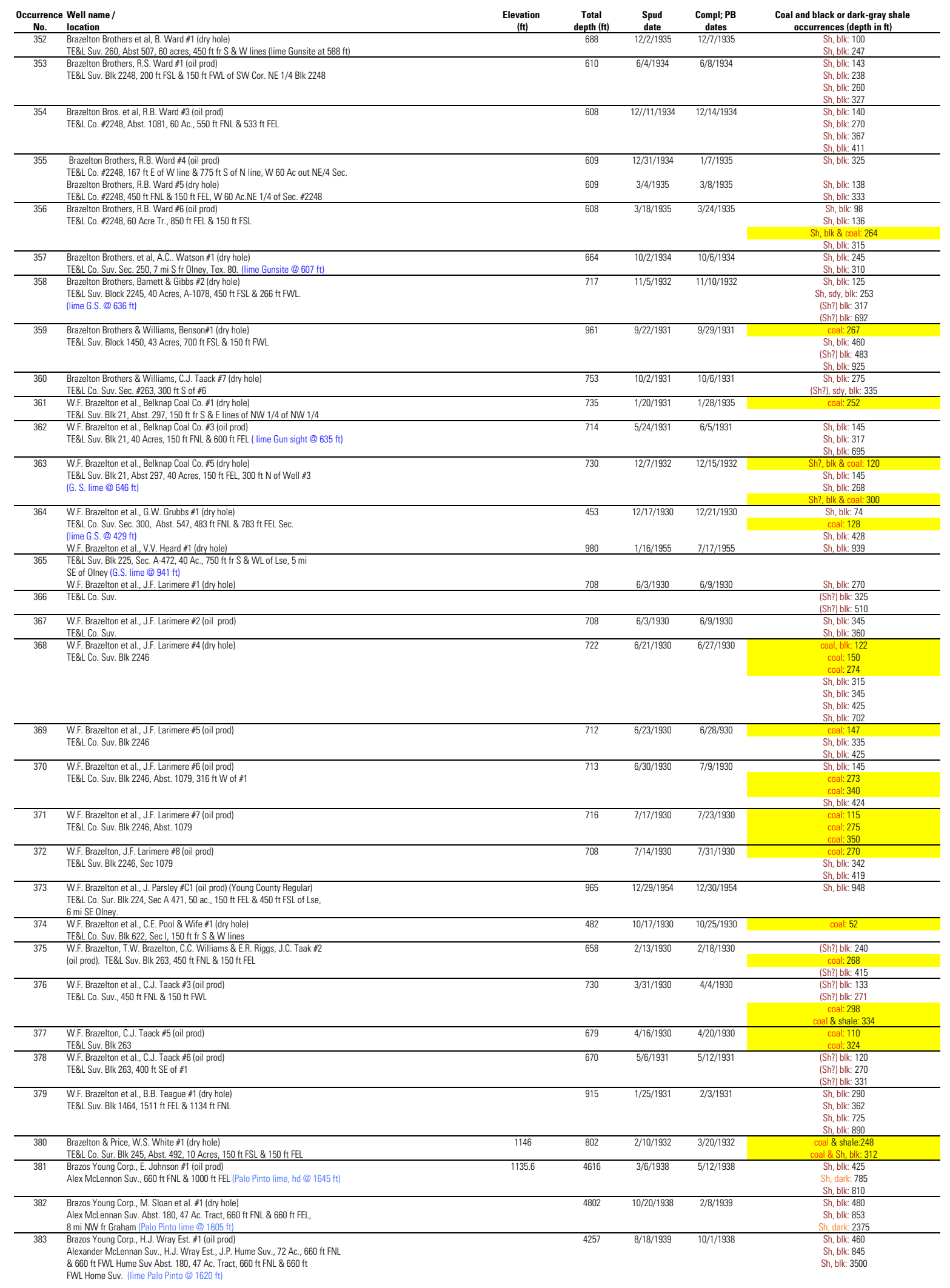




\begin{tabular}{|c|c|c|c|c|c|c|}
\hline $\begin{array}{l}\text { Occurrence } \\
\text { No. }\end{array}$ & $\begin{array}{l}\text { Well name / } \\
\text { location }\end{array}$ & $\begin{array}{c}\text { Elevation } \\
\text { (ft) }\end{array}$ & $\begin{array}{c}\text { Total } \\
\text { depth (ft) }\end{array}$ & $\begin{array}{l}\text { Spud } \\
\text { date }\end{array}$ & $\begin{array}{c}\text { Compl; PB } \\
\text { dates }\end{array}$ & $\begin{array}{l}\text { Coal and black or dark-gray shale } \\
\text { occurrences (depth in ft) }\end{array}$ \\
\hline 384 & $\begin{array}{l}\text { Bredshall Dil Co., W.F. Bailey \#1 (dry hole) } \\
\text { TE\&L Co. Suv. Blk 26, Abst } 306,87 \text { Acres, } 450 \text { ft FEL \& } 1050 \mathrm{ft} \text { FSL } \\
\text { of Suv. A-302 (lime, Gunshight @ } 773.5 \mathrm{ft} \text { ) }\end{array}$ & & 902 & 9/10/1951 & 9/14/1951 & $\begin{array}{l}\text { Sh \& coal: } 240 \\
\text { Sh \& coal: } 375 \\
\text { Sh, blk: } 773\end{array}$ \\
\hline 385 & $\begin{array}{l}\text { Bredshall Oil Co., A. Lemerman \#1 (dry hole) (Killian field) } \\
\text { TE\&L Co. Suv. Sec. } 220,731 / 3 \text { Ac., } 150 \mathrm{ft} \mathrm{FSL} \mathrm{\&} 750 \mathrm{ft} \text { FEL, } 3 \text { mi S fr Onley }\end{array}$ & 1200 & 980 & $9 / 20 / 1951$ & $9 / 24 / 1951$ & Sh, blk: 927 \\
\hline & $\begin{array}{l}\text { Brek Oil Co., C.F. Newman \#2 (oil prod) } \\
\text { Susan Sallie Suv. } 40 \text { Ac., } 300 \mathrm{ft} \text { Eof \#1, thence } \mathrm{N} 150 \mathrm{ft} t \text { location of well }\end{array}$ & & 550 & $12 / 26 / 1933$ & $1 / 3 / 1934$ & $\begin{array}{c}\text { coal: } 31 \\
\text { Sh, blk: } 115 \\
\text { coal: } 140 \\
\text { coal: } 215 \\
\end{array}$ \\
\hline 386 & $\begin{array}{l}\text { Brek Oil Co.. C.F. Newman \# \# (oil prod) } \\
\text { Susan Sallie Suv., } 40 \text { Ac., appr. } 450 \mathrm{ft} F W L \text { \& appr. } 400 \mathrm{ft} F S L\end{array}$ & & 543 & $2 / 20 / 1934$ & $3 / 1 / 1934$ & $\begin{array}{l}\text { coal: } 26 \\
\text { Sh, blk: } 110 \\
\text { coal: } 135 \\
\text { coal: } 210\end{array}$ \\
\hline 387 & $\begin{array}{l}\text { Bridwell Oil Co., J.M. Bloodworth \#1 (dry hole) } \\
\text { TE\&L Co. Suv., Blk } 326,150 \mathrm{ft} \text { fr W, } 450 \mathrm{ft} \text { fr N line Sec. }\end{array}$ & & 1055 & 10/29/1930 & 11/8/1930 & $\begin{array}{l}\text { Sh, blk: } 410 \\
\text { Sh, blk: } 511 \\
\text { Sh, blk: } 598 \\
\text { Sh, blk: } 955\end{array}$ \\
\hline 388 & $\begin{array}{l}\text { Bridwell 0il Co., Bridwell \& Mayfield \#2 (oil prod) (WC) } \\
\text { A. Bryant Abst. 1950, } 330 \mathrm{ft} F \mathrm{FL} \& 467 \mathrm{ft} \text { FWL of Ise, } 237 \mathrm{ft} \text { of \#1, } \\
8 \text { mi ESE fr Graham }\end{array}$ & & 2709.5 & 9/16/1944 & $11 / 1 / 2 / 1944$ & $\begin{array}{l}\text { Sh, blk: } 1435 \\
\text { Sh, blk: } 1954\end{array}$ \\
\hline 389 & $\begin{array}{l}\text { Bridwell Dil Co., R.J. Calvin \#1 (dry hole) (Markley North-Strawn-field) } \\
\text { A. Rouhus Sur A-240, } 25 \text { ac., } 330 \mathrm{ft} f \mathrm{r} \text { \& EL, } 3 \text { mi N Markley }\end{array}$ & & 3642 & $10 / 7 / 1952$ & $10 / 21 / 1952$ & coal: 2267 \\
\hline 390 & $\begin{array}{l}\text { Bridwell Oil Co., G.W. Christian \#1 (dry hole) } \\
\text { TE\&L Co. Suv., Blk } 255,72 \text { Acres, } 850 \mathrm{ft} N \& 800 \mathrm{ft} \text { W of SW Corner }\end{array}$ & & 817 & 1/22/1935 & 1/29/1935 & $\begin{array}{c}\text { coal: } 230 \\
\text { Sh, sdy \& coal: } 316 \\
\text { Sh, llk: } 562 \\
\text { Sh, dark: } 648 \\
\text { Sh, dark gy: } 772 \\
\text { Sh, blk: } 779 \\
\text { Sh, dark: } 797\end{array}$ \\
\hline 391 & $\begin{array}{l}\text { Bridwell 0il Co., F.M. Cullers \#1 (oil prod) } \\
\text { YCSL Sur Blk 25, } 150 \mathrm{ft} \text { fr N \& W lines of Blk } 25\end{array}$ & & 618.5 & $5 / 16 / 1929$ & $5 / 23 / 1929$ & Sh, blk: 150 \\
\hline 392 & $\begin{array}{l}\text { Bridwell 0il Co., F.M. Cullers \#1 (oil prod) } \\
\text { J.L. Hytt Suv.. } 450 \mathrm{ft} \text { W \& } 150 \mathrm{ft} \text { S of NE Cor. Of Suv. } 40 \text { Ac., Abst. } 2166\end{array}$ & & 613 & $8 / 8 / 1933$ & 8/13/1933 & $\begin{array}{c}\text { Sh, blk: } 114 \\
\text { Sh, blk: } 128 \\
\text { coal: } 150 \\
\text { Sh, blk: } 197 \\
\text { Sh, blk: } 528\end{array}$ \\
\hline 393 & $\begin{array}{l}\text { Bridwell 0il Co., F.M. Cullers \#1 (oil prod) (WC) } \\
\text { J.L. Hyatt A-2166 Surv., } 160 \text { Acres, } 330 \mathrm{ft} \text { FEL \& } 1263 \mathrm{ft} \text { FSL of Sec } 2 \text {, } \\
3 \text { mi NW fr Markley }\end{array}$ & & 3680 & 11/28/1951 & $12 / 11 / 1951$ & $\begin{array}{l}\text { coal: } 140 \\
\text { Sh \& coal: } 2359\end{array}$ \\
\hline 394 & $\begin{array}{l}\text { Bridwell Oil Co., F.M. Cullers \#2 (oil prod) } \\
\text { YCSL Survey Blk 25, } 310 \mathrm{ft} \text { NL \& } 500 \mathrm{ft} \text { WL }\end{array}$ & & 604 & 6/21/1929 & 6/28/1929 & Sh, blk: 145 \\
\hline 395 & $\begin{array}{l}\text { Bridwell Oil Co., F.M. Cullers \#3 (dry hole) } \\
\text { Suv., } 890 \text { fr WL \& } 575 \mathrm{ft} \text { NL of Lse }\end{array}$ & & 620 & $7 / 3 / 1929$ & $7 / 8 / 1929$ & $\begin{array}{l}\text { Sh, blk: } 135 \\
\text { Sh, blk: } 472 \\
\text { Sh, blk: } 602\end{array}$ \\
\hline 396 & $\begin{array}{l}\text { Bridwell Oil Co., J.B. Daniels \#4 (dry hole) } \\
\text { TE\&L Co. Suv. Blk } 164,40 \text { Acre Tract, } 600 \mathrm{ft} \text { W of E line \& } 466 \mathrm{ft} \mathrm{fr} N \& \text { S lines }\end{array}$ & & 937 & $1 / 131 / 1935$ & $2 / 7 / 1935$ & Sh, blk: 873.7 \\
\hline 397 & $\begin{array}{l}\text { Bridwell Oil Co., S.A. Haley \#1 (dry hole) } \\
\text { TE\&L Co. Suv. Blk 293, } 1716 \text { ft fr N \& EL }\end{array}$ & 11157 DF & 925 & $5 / 22 / 1930$ & $5 / 31 / 1930$ & $\begin{array}{l}\text { Sh, blk: } 196 \\
\text { Sh, blk: } 257 \\
\text { Sh, blk: } 571 \\
\text { Sh, blk: } 770\end{array}$ \\
\hline 398 & $\begin{array}{l}\text { Bridwell Oil Co., J.G. Hawlins \#1 (dry hole) } \\
\text { TE\&L Co. Suv. Blk } 1917,40 \text { Acres, } 150 \text { ft FS \& W line of } 40 \text { Acre Tract }\end{array}$ & 1188 & 600 & 1/30/1932 & $2 / 3 / 1932$ & $\begin{array}{l}\text { Sh, blk: } 135 \\
\text { Sh, blk: } 483\end{array}$ \\
\hline 399 & $\begin{array}{l}\text { Bridwell Oil Co., J.A. Leberman \#1 (dry hole) } \\
\text { EE\&L Co. Suv. Blk } 151,627 \mathrm{ft} \text { SL \& } 1737 \mathrm{ft} \text { EL Surv. }\end{array}$ & 1201.9 & 970 & $2 / 18 / 1930$ & $3 / 3 / 1930$ & $\begin{array}{l}\text { Sh, blk: } 454 \\
\text { Sh, blk: } 864\end{array}$ \\
\hline 400 & $\begin{array}{l}\text { Bridwell Oil Co., E.R. \& H.O. Prideaux \#1 (dry hole) } \\
\text { CTRR Co. Survey Blk. 1, } 150 \text { ft fr N \& E Lines }\end{array}$ & & 736 & $5 / 30 / 1929$ & $6 / 7 / 1929$ & Sh, blk: 136 \\
\hline 401 & $\begin{array}{l}\text { Bridwell 0il Co., H.O. Prideaux " "B" Est \#1 (dry hole) (Markley N-Strawn field) } \\
\text { Assoose Rohus A-240 Sur., } 330 \mathrm{ft} \text { fr S \& EL, } 4 \text { mi N fr Markley }\end{array}$ & & 3706 & $5 / 21 / 1953$ & 6/2/1953 & $\begin{array}{l}\text { Sh \& coal: } 2282 \\
\text { Sh \& coal: } 2395\end{array}$ \\
\hline 402 & 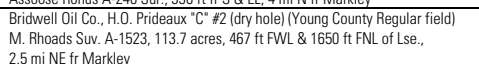 & & & & & Sh, blk, soft \& lime: 2505 \\
\hline 403 & $\begin{array}{l}\text { Bridwell Dil Co., Prideaux-Sams \#1 (dry hole) (WC) } \\
\text { Young Co Sch Lnd A-1284 Sur., Sec 27, } 80 \text { Ac., } 330 \mathrm{ft} \text { FNL \& } 330 \mathrm{ft} \text { FWL of Lse, } \\
1.5 \mathrm{mi} \text { N fr Markley }\end{array}$ & & 3680 & $4 / 25 / 1953$ & $5 / 5 / 1953$ & Sh \& coal: 232 \\
\hline 404 & $\begin{array}{l}\text { Bridwell Oil Co., Prideaux-Sikes \#1 (oil prod) (Prideaux } 3000 \mathrm{ft} \text { field) } \\
\text { Assoose Rohus A-240 Sur., Blk 5, } 80 \text { ac., } 330 \mathrm{ft} \text { FWL \& } 1263 \mathrm{ft} \text { FNL }\end{array}$ & & 3040 & $5 / 12 / 1953$ & $5 / 20 / 1953$ & Sh \& coal: 268 \\
\hline 405 & $\begin{array}{l}\text { Bridwell 0il Co., Prideaux-Sikes \#2 (oil prod)] (Prideaux Strawn } 3000 \mathrm{ft} \text { field) } \\
\text { Assoose Rohus A-240 Sur., Blk } 5,80 \text { ac., } 330 \mathrm{ft} \text { FEL \& } 819 \mathrm{ft} \text { FSL }\end{array}$ & & 3049 & 6/5/1953 & $6 / 12 / 1953$ & Sh \& coal: 198 \\
\hline 406 & $\begin{array}{l}\text { Bridwell Oil Co., Prideaux-Sikes \#3 (dry hole) (Prideaux Strawn } 3000 \mathrm{ft} \mathrm{field)} \\
\text { Assoose Rohus A-240 Sur., Blk 5, } 80 \text { Ac., } 330 \mathrm{ft} F S L \text { \& } 773 \mathrm{ft} \mathrm{FEL} \mathrm{of} \mathrm{Lse,} \\
3 \mathrm{mi} \text { NW fr Markley }\end{array}$ & & 3700 & 7/14/1953 & $7 / 28 / 1953$ & Sh \& coal: 190 \\
\hline 407 & $\begin{array}{l}\text { Bridwell Oil Co., T.J. Routon \#1 (dry hole) } \\
\text { TE\&L Suv. Blk. } 24,45 \text { Acre Tr., } 200 \mathrm{ft} \mathrm{FNLL} \mathrm{\&} 700 \mathrm{ft} \text { FEL }\end{array}$ & & 803 & $1 / 31 / 1932$ & $2 / 9 / 1932$ & $\begin{array}{l}\text { coal: } 120 \\
\text { Sh, blk: } 330 \\
\text { Sh, blk: } 340 \\
\text { Sh, blk: } 663\end{array}$ \\
\hline 408 & $\begin{array}{l}\text { Bridwell 0il Co., S.E. Snider \#2 (dry hole) } \\
\text { R Wilson Sur. A-1276, } 153 \text { ac., } 330 \mathrm{ft} \text { FNL \& } 1263 \mathrm{ft} \text { FEL of Lse, } 4 \text { mi N Markley }\end{array}$ & & 3650 & 12/14/1951 & 12/30/1951 & $\begin{array}{l}\text { Sh; s; \& coal strk: } 83 \\
\text { Sh; s; \& coal strk: } 303 \\
\text { Sh, blk, soft: } 2289\end{array}$ \\
\hline 409 & $\begin{array}{l}\text { Bridwell Oil Co., S.E. Snider \#4 (oil prod) (Markley N Strawn field) } \\
\text { R Wilson Sur. A-1276, } 414.14 \text { ac., } 1677 \mathrm{ft} \mathrm{FNL} \mathrm{\&} 1711 \mathrm{ft} \mathrm{FWL}\end{array}$ & & 3626 & $4 / 8 / 1952$ & $4 / 18 / 1952$ & coal: 335 \\
\hline 410 & $\begin{array}{l}\text { Bridwell Oil Co., S.E. Snider } \# 6 \text { (oil prod)(Markley N Strawn field) } \\
\text { R Wilson Sur. A-1276, } 415 \text { ac., } 990 \mathrm{ft} \text { FNL \& } 330 \mathrm{ft} \text { FWL }\end{array}$ & & 3620 & 8/29/1952 & 9/10/1952 & Sh, blk: 133 \\
\hline 411 & $\begin{array}{l}\text { Bridwell Oil Co., S.E. Snider \#9 (oil prod) (Markley N Strawn field) } \\
\text { R Wilson Sur. A-1276, } 415.14 \text { ac. } 467 \mathrm{ft} F E L \text { \& } 1252 \mathrm{ft} \text { FNL of Lse. }\end{array}$ & & 3618 & $6 / 5 / 1956$ & $6 / 22 / 1956$ & coal: 225 \\
\hline 412 & $\begin{array}{l}\text { Bridwell Oil Co., S.E. Stowe \#1 (dry hole) } \\
\text { TE\&L Co. Suv., Blk 228, } 1050 \mathrm{ft} \text { FNL \& } 150 \mathrm{fr} \text { FWL Sec. }\end{array}$ & $1188 \mathrm{DF}$ & 1085 & $6 / 4 / 1930$ & 6/13/1930 & $\begin{array}{l}\text { Sh, blk: } 344 \\
\text { Sh, blk: } 532 \\
\text { Sh, blk: } 992\end{array}$ \\
\hline 413 & $\begin{array}{l}\text { Bridwell Oil Co., A. Williams \#1 (oil prod) (Cullers field) } \\
\text { Young Co. School Ld BIi 26, } 110 \text { Ac., } 150 \mathrm{ft} f \mathrm{fr} \& \text { \& W lines }\end{array}$ & & 633 & & 8/24/1938 & $\begin{array}{l}\text { Sh, bll: } 205 \\
\text { Sht, blk: } 559 \\
\text { Sh, blk: } 592\end{array}$ \\
\hline 414 & $\begin{array}{l}\text { Bridwell Oil Co., A. Williams \#2 (oil prod) (Cullers field) } \\
\text { Young Co. School Ld Suv., Blk 26, } 110 \text { Ac., } 150 \mathrm{ft} \mathrm{FWL} \mathrm{\&} 450 \mathrm{fr} \text { FSL }\end{array}$ & & 631 & & $8 / 24 / 1938$ & $\begin{array}{l}\text { Sh, blk: } 150 \\
\text { Sh, blk: } 564\end{array}$ \\
\hline 415 & $\begin{array}{l}\text { Bridwell Oil Co., A. Williams \#3 (oil prod) } \\
\text { Young Co. Sch Ld Suv., Blk 26, } 110 \text { Ac., } 150 \mathrm{ft} \mathrm{FWL} \mathrm{\&} 750 \mathrm{fr} \text { FSL, } 300 \mathrm{ft} N \text { of \#2 }\end{array}$ & & 655 & $7 / 29 / 1938$ & $8 / 3 / 1938$ & $\begin{array}{l}\text { Sh, blk: } 165 \\
\text { Sh, blk: } 585\end{array}$ \\
\hline 416 & $\begin{array}{l}\text { Bridwell Oil Co., F.M. Cullers \#1 (oil prod) } \\
\text { J.L. Hyatt Suv., Abst \#2166, } 150 \mathrm{ft} \text { \& \& } 150 \mathrm{ft} \text { W of NE Cor. SW } 40 \text { Ac. }\end{array}$ & 1040.4 & 627 & $9 / 5 / 1929$ & 9/12/1929 & Sh, blk: 545 \\
\hline 417 & $\begin{array}{l}\text { Bridwell \& Mayfield, F.M. Cullers \#2 (oil prod) } \\
\text { J.L. Hyatt Abst } 2156,15 \mathrm{ft} S \& 150 \mathrm{ft} \text { Eof NW Cor. of SW } 40 \text { Ac. }\end{array}$ & 1036 & 628 & 9/15/1929 & 9/22/1929 & Sh, blk: 115 \\
\hline 418 & $\begin{array}{l}\text { Bridwell \& Mayfield, F.M. Cullers \#3 (oil prod) } \\
\text { J.L. Hyatt Suv. Abst \#2166, } 150 \mathrm{ft} S \& 486 \mathrm{ft} \text { of NW Cor. of SW } 40 \mathrm{Ac} \text {. }\end{array}$ & 1039 & 606 & 9/27/1929 & 10/2/1929 & $\begin{array}{l}\text { Sh, blk: } 125 \\
\text { Sh, blk: } 287 \\
\text { Sh, blk: } 546\end{array}$ \\
\hline 419 & $\begin{array}{l}\text { Bridwell \& Mayfield, F.M. Cullers } \# 6 \text { (dry hole) } \\
\text { J.L. Hyatt Suv. Abst } 2166,498 \mathrm{ft} S \& 486 \mathrm{ft} \text { E of NW Cor of SW } 40 \text { Acres }\end{array}$ & & 722 & 12/4/1929 & 12/9/1929 & $\begin{array}{l}\text { Sh, blk: } 121 \\
\text { Sh, blk: } 556\end{array}$ \\
\hline
\end{tabular}




\begin{tabular}{|c|c|c|c|c|c|c|}
\hline $\begin{array}{l}\text { Occurrence } \\
\text { No. }\end{array}$ & $\begin{array}{l}\text { Well name / } \\
\text { location }\end{array}$ & $\begin{array}{c}\text { Elevation } \\
\text { (ft) }\end{array}$ & $\begin{array}{c}\text { Total } \\
\text { depth (ft) }\end{array}$ & $\begin{array}{l}\text { Spud } \\
\text { date }\end{array}$ & $\begin{array}{c}\text { Compl; PB } \\
\text { dates }\end{array}$ & $\begin{array}{l}\text { Coal and black or dark-gray shale } \\
\text { occurrences (depth in ft) }\end{array}$ \\
\hline 420 & $\begin{array}{l}\text { Bridwell Oil Co. \& Petroleum Prod. Co... J.B. Daniel \#1 (oil prod) } \\
\text { TE\&L Co. Suv., Blk 164, } 1083 \mathrm{ft} \text { fr S \& } 1221 \mathrm{ft} \text { fr E L }\end{array}$ & & 901 & $3 / 23 / 1930$ & $4 / 1 / 1930$ & $\begin{array}{l}\text { Sh, blk: } 434 \\
\text { Sh, blk: } 448 \\
\text { Sh, blk: } 489\end{array}$ \\
\hline 421 & $\begin{array}{l}\text { Bridwell 0il Co. \& Petroleum Prod. Co., J.B. Daniel \#2 (dry hole) } \\
\text { TE\&L Co. Suv., BIK } 164,150 \mathrm{ft} \text { fr N \& W lines }\end{array}$ & & 922 & 4/4/1930 & 4/15/1930 & $\begin{array}{l}\text { Sh, blk: } 315 \\
\text { Sh, blk: } 453\end{array}$ \\
\hline 422 & $\begin{array}{l}\text { Bridwell Oil Co. \& \& Petroleum Prod. Co., J.B. Daniel \#3 (oil prod) } \\
\text { TE\&\& Co. Suv., Blk } 164\end{array}$ & & 915 & 4/21/1930 & 4/29/1930 & $\begin{array}{l}\text { Sh, blk: } 449 \\
\text { Sh, blk: } 898\end{array}$ \\
\hline 423 & $\begin{array}{l}\text { Bridwell 0il Co., J.W. Daws \#1 (oil prod) (WC) } \\
\text { TE\&L Co. Suv., Sec 765, } 320 \text { Ac., } 4 \text { mi W fr Proffitt }\end{array}$ & & 1909 & 3/17/1943 & 3/26/1943 & $\begin{array}{c}\text { coal: } 363 \\
\text { coal: } 475 \\
\text { Sh, blk: } 516 \\
\text { Sh, blk: } 536 \\
\text { coal: } 542 \\
\text { Sh, blk: } 1046 \\
\text { Sh, blk: } 1358 \\
\text { coal: } 1440\end{array}$ \\
\hline 424 & $\begin{array}{l}\text { Bridwell 0il Co., J.W. Daws "A" \#1 (dry hole) } \\
\text { TE\&L Co. Suv., Sec 764, } 180 \text { Acres, } 330 \mathrm{ft} \text { FNL \& } 934 \mathrm{ft} \text { FWL, } 4 \text { mi W fr Proffitt }\end{array}$ & & 991 & 3/27/1943 & 4/1/1943 & $\begin{array}{l}\text { coal: } 374 \\
\text { coal: } 486 \\
\text { coal: } 525 \\
\text { coal: } 547 \\
\text { coal: } 551 \\
\text { Sh, blk: } 861\end{array}$ \\
\hline 425 & $\begin{array}{l}\text { Bridwell Oil Co., B.J. Hulse et al \#A-1 (dry hole) } \\
\text { TE\&L Co. Suv., Sec } 767,100 \text { Ac., } 300 \mathrm{ft} \mathrm{FNL} \mathrm{\&} 1166 \mathrm{ft} \mathrm{FEL,} 1167 \mathrm{ft} f \mathrm{fr} \text { WL } \\
\text { A } 100 \text { Ac. Tr., } 4 \text { mi W fr Profffitt }\end{array}$ & & 2005 & 4/5/1943 & 4/11/1943 & $\begin{array}{l}\text { coal: } 411 \\
\text { coal: } 528 \\
\text { coali: } 575 \\
\text { coal: } 603 \\
\text { coal: } 982 \\
\text { Sh, blk: } 1459\end{array}$ \\
\hline 426 & $\begin{array}{l}\text { Bridwell 0il Co., D.L. Wolf \#1 (dry hole) } \\
\text { TE\&L Co. Suv., Blk } 165,572 \mathrm{ft} \text { NL \& } 150 \mathrm{ft} \text { EL of Sec. }\end{array}$ & $1186 \mathrm{DF}$ & 1003 & $12 / 27 / 1929$ & $1 / 6 / 1930$ & $\begin{array}{c}\text { Sh, blk: } 440 \\
\text { coal: } 517 \\
\text { Sh, blk: } 856 \\
\end{array}$ \\
\hline 427 & $\begin{array}{l}\text { Bridwell 0il Co. \& Petroleum Prod. Co., D.L. Wolf \#2 (oil prod) } \\
\text { TE\&L Co. Suv., Blk } 165,783 \mathrm{ft} \text { W \& } 850 \mathrm{ft} \text { S of NE Corner of Blk } 165 \\
\text { Bridwell Oil Co. \& E.J. Stump, T.J. Routen \# \#1 (dry hole) } \\
\text { TE\&L Co. Suv., Blk 181, } 1250 \mathrm{ft} \text { fr S \& } 150 \mathrm{ft} f \mathrm{fr} \text { WL Sec }\end{array}$ & & $\begin{array}{l}962 \\
947\end{array}$ & $\begin{array}{l}4 / 4 / 1932 \\
5 / 3 / 1930\end{array}$ & $\begin{array}{l}/ 26 / 1932 \\
5 / 14 / 1930\end{array}$ & $\begin{array}{l}\text { Sh, blk \& coal: } 309 \\
\text { Sh, bl: } 867 \\
\text { Sh, blk: } 319 \\
\text { Sh, blk: } 473 \\
\text { Sh, blk: } 550 \\
\text { Sh, blk: } 840\end{array}$ \\
\hline 428 & $\begin{array}{l}\text { Brihill Oil Corp., Peter Heirs \#1 (oil prod) } \\
\text { H.F. Peters Suv., } 150 \mathrm{ft} \text { fr } \& \text { \& W lines }\end{array}$ & 1108 & 740 & $4 / 1 / 1930$ & 4/15/1930 & coal: 225 \\
\hline 429 & $\begin{array}{l}\text { Brihill Oil Corp., R.D. Webb \#1 (dry hole) } \\
\text { Assoose Rehus Suv., } 150 \mathrm{ft} f \mathrm{fr} \text { \& E lines }\end{array}$ & 1121 & 827 & 2/25/1930 & 3/14/1930 & $\begin{array}{l}\text { Sh, blk: } 280 \\
\text { Sh, blk: } 691\end{array}$ \\
\hline 430 & $\begin{array}{l}\text { Brihill Oil Corp. \& L.G. Bradstreet Co., C.W. Matthews \#1 (dry hole) } \\
\text { A. Rohus Suv., } 1000 \mathrm{ft} \text { EL \& } 2035 \mathrm{ft} \text { SL }\end{array}$ & 1154 & 789 & $8 / 8 / 1929$ & 8/17/1929 & $\begin{array}{l}\text { Sh, blk: } 280 \\
\text { Sh, blk: } 370 \\
\text { Sh, blk: } 720\end{array}$ \\
\hline 431 & $\begin{array}{l}\text { Brihill Oil Corp. \& L.G. Bradstreet Co.. L.E. Self \#1 (dry hole) } \\
\text { Assoose Rohus Sur., } 150 \mathrm{ft} f \mathrm{fr} \text { \& \& Lines }\end{array}$ & 1143 & 775 & $7 / 4 / 1929$ & $7 / 17 / 1929$ & $\begin{array}{l}\text { (Sh?) blk: } 300 \\
\text { coal: } 371 \\
\text { Sh, blk:712 }\end{array}$ \\
\hline 432 & $\begin{array}{l}\text { The British American Oil Producing Co., M. Barrett \#1 (oil prod) } \\
\text { E.W. Sargent Suvv., } 86.25 \text { Ac. Lse., } 330 \mathrm{ft} N \text { of SL \& } 330 \mathrm{ft} W \text { of EL }\end{array}$ & 1225 & 5530 & $7 / 16 / 1943$ & 8/28/1943 & coal: 172 \\
\hline 433 & $\begin{array}{l}\text { H.R. Britton, Morrison-Kindley \#2 (WC) } \\
\text { TE\&L Co. Sur. \#431 \& 432, A } 638 \text { \& A 639, } 80 \text { ac, } 27 \text { ft FNL \& } 1546 \text { FWL of TE\&L Sur } 432 \\
5.5 \text { mi E Newcastle }\end{array}$ & & 4750 & $7 / 6 / 1961$ & 7/19/1961 & $\begin{array}{l}\text { Sh, blk: } 790 \\
\text { Sh, blk: } 3652 \\
\text { coal: } 4241 \\
\text { coal: } 4369\end{array}$ \\
\hline 434 & $\begin{array}{l}\text { E.C. Brown Trustee, L. Stewart \#1 (dry hole) (WC) } \\
\text { Tex Emigration Lds Sur., Sec } 229,212 \text { ac., } 330 \mathrm{ft} \text { FNL \& } 330 \mathrm{fr} \text { FWL of Lsse, } 2 \text { mi E Olney }\end{array}$ & & 3709 & 3/8/1953 & 3/25/1953 & coal: 598 \\
\hline 435 & $\begin{array}{l}\text { R.J. Brown, W.H. Daily \#1 (dry hole) } \\
\text { Sylphia Tynes Survey } 5,150 \mathrm{ft} E \text { and } 3105 \mathrm{ft} \mathrm{S} \text { of NW Corner of Survey; or } 150 \mathrm{ft} \text { of W \& } \\
150 \mathrm{ft} N \text { of S Lines }\end{array}$ & & 600 & 6/28/1928 & $7 / 6 / 1928$ & $\begin{array}{l}\text { Sh, blk: } 70 \\
\text { Sh, blk: } 143\end{array}$ \\
\hline 436 & $\begin{array}{l}\text { R.J. Brown \& Co., J.W. Hawkins et al \#1 (dry hole) } \\
\text { GB\&N Company Survey, Sec 2, } 150 \mathrm{ft} W \text { of E \& } 840 \mathrm{ft} N \text { of } S \text { Lines of } 70 \text { Acre Tract }\end{array}$ & & 720 & $5 / 4 / 1928$ & 5/25/1928 & $\begin{array}{l}\text { slatey coal: } 150 \\
\text { Sh, blk: } 215 \\
\text { Sh, blk: } 273 \\
\text { Sh, dark } 646\end{array}$ \\
\hline 437 & $\begin{array}{l}\text { G.G. Browning, C.E. Howard \#1 (dry hole) } \\
\text { TE\&L Co. Suv., \#243, Abst. \# 490, } 150 \mathrm{ft} W \text { of E Line \& } 450 \mathrm{ft} N \text { fr } S \text { Line of East } 50 \text { Acres } \\
\text { of N. } 160 \text { Ac. }\end{array}$ & & 735 & 10/12/1932 & $10 / 22 / 1932$ & $\begin{array}{l}\text { Sh, blk: } 104 \\
\text { Sh, blk; coal: } 280 \\
\text { Sh, blk: } 335\end{array}$ \\
\hline 438 & $\begin{array}{l}\text { A.H. Bruner \& Co., B.W. King \#1 (oil prod) (Garvey Strawn field) } \\
\text { Susan Sllee Sur. A-252, } 330 \mathrm{ft} \text { FWL \& } 660 \mathrm{ft} \text { FSL of } 75 \text { ac. }\end{array}$ & 1190 & 3488 & 4/4/1955 & 4/13/1955 & coal: 125 \\
\hline 439 & $\begin{array}{l}\text { R. Bruton, S.H. Jones \#1 (dry hole) } \\
\text { Abst. 40, Block 1, } 80 \text { arres, } 750 \text { ft FNL \& } 150 \mathrm{ft} \text { FEL }\end{array}$ & & 673 & 10/6/1932 & 10/22/1932 & $\begin{array}{l}\text { Sh, blk: } 137 \\
\text { Sh, blk: } 223 \\
\text { Sh, blk: } 477 \\
\text { Sh, blk: } 595\end{array}$ \\
\hline 440 & $\begin{array}{l}\text { Bryson Oil \& Gas Co., J.M. Nall \#1 (oil prod) } \\
\text { TE\&LL Co. Suv., Blk } 1997\end{array}$ & 1271 & 4465 & 11/26/1938 & $6 / 4 / 1939$ & $\begin{array}{l}\text { coul: } 192 \\
\text { Sh, blk: } 925\end{array}$ \\
\hline 441 & $\begin{array}{l}\text { D. Buckalew et al., L.J. Kilcrease \#2 (oil prod) (Young County Regular field) } \\
\text { TE\&L Co. Block \#1809, Sec. } 971,40 \text { ac., } 750 \mathrm{ft} \text { FSL \& } 450 \mathrm{ft} F W L\end{array}$ & 1215 & 1009 & $3 / 1 / 1951$ & $3 / 5 / 1951$ & coal: 563 \\
\hline 442 & $\begin{array}{l}\text { Bullington, Lee, Pendleton \& Bullington, F.L. Booone } \# 7 \text { (oil prod) } \\
\text { BBB\&C Ry Co. Sur., Sec } 1,80 \text { acre tract, } 150 \mathrm{ft} E \text { of } W \& 750 \mathrm{ft} S \text { of } \mathrm{N} \text { lines }\end{array}$ & & 545 & 1/16/1929 & 1/26/1929 & $\begin{array}{l}\text { coal: } 158 \\
\text { Sh, blk: } 394 \\
\text { Sh, blk: } 518\end{array}$ \\
\hline 443 & $\begin{array}{l}\text { Bullington, Lee, Pendleton \& Bullington, J.L. Boone \#3 (oil prod) } \\
\text { BBB\&C Ry Co. Suv. Sec \#1, } 80 \text { acre tract, } 570 \mathrm{ft} S \mathrm{SL} \& 30 \mathrm{ft} \text { WL }\end{array}$ & & 500 & 4/27/1928 & $5 / 7 / 1928$ & $\begin{array}{l}\text { Sh, blk: } 98 \\
\text { Sh, blk: } 167 \\
\text { Sh, blk: } 459\end{array}$ \\
\hline 444 & $\begin{array}{l}\text { Bullington, Lee, Pendleton \& Bullington, J.L. Boone \#4 (oil prod) } \\
80 \text { acre tract, } 450 \mathrm{ft} E \text { of } W \text { line, appx. } 150 \mathrm{ft} f \mathrm{fr} \text { S line }\end{array}$ & & 493.5 & $5 / 10 / 1928$ & 5/16/1928 & $\begin{array}{l}\text { Sh, blk: } 98 \\
\text { Sh, blk: } 167\end{array}$ \\
\hline 445 & $\begin{array}{l}\text { Bullington, Lee, Pendleton \& Bullington, J.L. Boone \#5 (oil prod) } \\
\text { BBB\&C RR Surv., Sec } 1,80 \text { Acres, } 500 \mathrm{ft} E \text { of } \mathrm{W}, 750 \mathrm{ft} S \text { of } \mathrm{N} \text { lines }\end{array}$ & & 497 & $5 / 28 / 1928$ & 6/4/1928 & coal: 95 \\
\hline 446 & $\begin{array}{l}\text { Bullington, Lee, Pendleton \& Bullington, J.L. Boone \#6 (oil prod) } \\
\text { BBB\&C RR Surv., Sec } 1,80 \text { A. Tract, } 740 \mathrm{ft} \text { of W, } 150 \mathrm{ft} N \text { of S lines, } \\
\text { appx. footage fr S line } 80 \text { A. Tract }\end{array}$ & & 496 & 6/11/1928 & 6/17/1928 & $\begin{array}{l}\text { coal: } 95 \\
\text { Sh, blk: } 463 \\
\text { Sh, blk: } 492\end{array}$ \\
\hline 447 & $\begin{array}{l}\text { R. Bullock, R. Bullock \#1 (dry hole) } \\
\text { Mary Hamilton Suv. Abs. } 138,230 \text { Acres, } 270 \mathrm{ft} \text { FSL \& } 221 \mathrm{ft} \text { FWL }\end{array}$ & & 462 & 1/12/1936 & $2 / 3 / 1936$ & $\begin{array}{l}\text { Sh, dark: } 448 \\
\text { Sh, blk: } 460\end{array}$ \\
\hline 448 & $\begin{array}{l}\text { R. Bullock, S.L. Scott \#1 (bol prod)(WC) } \\
\text { TE\&L Co. Sur. Blk } 345,80 \text { ac., } 200 \mathrm{ft} \text { FNL \& } 170 \mathrm{ft} \text { FEL, } 8 \text { mi NW Newcastle }\end{array}$ & & 747 & $4 / 7 / 1955$ & $4 / 26 / 1955$ & $\begin{array}{l}\text { Sh, blik: } 370 \\
\text { coal: } 401 \\
\text { Sh, blk: } 446 \\
\text { Sh, blk: } 741\end{array}$ \\
\hline 449 & $\begin{array}{l}\text { R. Bullock, S.L. Scott \#2 (oil prod) (Yung County Regular field) } \\
\text { TE\&L Surv., Blk. } 345,80 \text { ac., } 475 \mathrm{ft} \mathrm{FEL} \mathrm{\&} 335 \mathrm{ft} \text { FNL, } 300 \mathrm{ft} f \mathrm{fr} \# 1\end{array}$ & & 746 & $7 / 8 / 1955$ & $7 / 16 / 1955$ & $\begin{array}{l}\text { coal: } 290 \\
\text { coal: } 401 \\
\text { Sh, blk: } 445 \\
\text { Sh, blk: } 741\end{array}$ \\
\hline 450 & $\begin{array}{l}\text { J. Bundy et al., J.W. Bryan \#1 (dry hole) } \\
\text { TE\&L Co. Survey, Sec. } 1902\end{array}$ & & 753 & $11 / 19 / 1928$ & $11 / 22 / 1928$ & $\begin{array}{l}\text { coal Sh: } 291 \\
\text { Sh, bl: } 365 \\
\text { Sh, blk: } 743\end{array}$ \\
\hline 451 & $\begin{array}{l}\text { J. Bundy, J.B. Foster \#1 (dry hole) (Jean field) } \\
\text { TE\&\&L Suv., Blk } 620,40 \text { acres, } 150 \text { ft FNL \& } 933 \mathrm{ft} \mathrm{FEL}\end{array}$ & & 600 & 4/26/1936 & $5 / 6 / 1936$ & 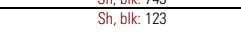 \\
\hline
\end{tabular}




\begin{tabular}{|c|c|c|c|c|c|c|}
\hline $\begin{array}{l}\text { Occurrence } \\
\text { No. }\end{array}$ & $\begin{array}{l}\text { e Well name / } \\
\text { location }\end{array}$ & $\begin{array}{c}\text { Elevation } \\
\text { (ft) }\end{array}$ & $\begin{array}{c}\text { Total } \\
\text { depth (ft) }\end{array}$ & $\begin{array}{l}\text { Spud } \\
\text { date }\end{array}$ & $\begin{array}{c}\text { Compl; PB } \\
\text { dates }\end{array}$ & $\begin{array}{l}\text { Coal and black or dark-gray shale } \\
\text { occurrences (depth in ft) }\end{array}$ \\
\hline 452 & $\begin{array}{l}\text { Burk Royalty Company, The Allar Company \#2 (dry hole) (Allar Hunt field) } \\
\text { TE\&L Co. Suv. Blk 3409, } 224 \text { Acres, } 467 \mathrm{ft} \mathrm{FEL,} 1008 \mathrm{ft} \text { N of \#1, } 10 \text { mi NE fr Southbend }\end{array}$ & 1135 Est. & 3607 & 4/3/1943 & 5/5/1943 & $\begin{array}{c}\text { coal: } 150 \\
\text { coal: } 246 \\
\text { Sh, blk, hd: } 2669\end{array}$ \\
\hline 453 & $\begin{array}{l}\text { Burk Royalty Company, W.D. Daws \#1 (dry hole) (WC) } \\
\text { TE\&LL Co. Suv. Sec } 753,320 \text { Acres, } 330 \mathrm{ft} \text { FEL \& } 330 \mathrm{ft} \text { FSL of Lse., } 6 \mathrm{mi} \text { SW fr Profitt }\end{array}$ & & 2912 & $5 / 8 / 1954$ & $5 / 16 / 1954$ & lignite: 244 \\
\hline 454 & $\begin{array}{l}\text { Burk Royalty Company, Hulse \&f (dry hole) [WC) } \\
\text { TE\&L Co. Suv. \#762, } 40 \text { Ac., } 330 \mathrm{ft} \text { FNL \& } 330 \mathrm{ft} \text { FEL of SW } 40 \text { Ac. of Suv., } 5 \mathrm{mi} \text { NW fr Proffitt }\end{array}$ & & 4889 & 5/22/1948 & 6/22/1948 & $\begin{array}{l}\text { coal: } 351 \\
\text { coal \& Sh: } 459 \\
\text { coal \& Sh: } 539\end{array}$ \\
\hline 455 & $\begin{array}{l}\text { Burk Royalty Company, McCloud \#3 (oil prod) (Sewell field) } \\
\text { BS\&F Suv., } 732 \mathrm{ft} \mathrm{fr} \mathrm{N} \& \mathrm{~W} \text { lines }\end{array}$ & & 2271 & 4/13/1947 & 4/25/1947 & Sh, blk: 1065 \\
\hline 456 & $\begin{array}{l}\text { Burlingame \& Mahan, C.C. Robinson \#1 (dry hole) } \\
\text { TE\&L Co. Suv. Abst. } 489, \text { Sec. } 1650,1690 \mathrm{ft} \text { FNL \& } 1740 \mathrm{ft} \text { FEL of Suv. }\end{array}$ & 1309 & 450 & 6/13/1938 & 6/17/1938 & $\begin{array}{l}\text { coal: } 274 \\
\text { Sh, blk: } 310 \\
\text { Sh, blk: } 448\end{array}$ \\
\hline 457 & $\begin{array}{l}\text { Burma Petroleum Co., J.P. Dunagan } \# 2 \text { ( oil prod)( (N Young County field) } \\
\text { TE\&L Co. Suv. Blk } 163,1700 \mathrm{ft} \mathrm{FEL} \mathrm{\&} 750 \mathrm{ft} F \mathrm{FL}\end{array}$ & & 919 & $3 / 10 / 1941$ & 3/19/1941 & Sh, blk: 919 \\
\hline 458 & $\begin{array}{l}\text { B.M. Burns, J.J. Perkins \#1 (dry hole) (Young County field) } \\
\text { TE\&L Co. Sur. Blk 252, A-499, } 40 \text { acres, } 330 \mathrm{ft} \text { \& } 330 \mathrm{ft} \text { of NW cor., } 2.5 \mathrm{mi} \text { E fr True }\end{array}$ & & 3202 & 1/16/1949 & $2 / 9 / 1949$ & coal: 239 \\
\hline 459 & $\begin{array}{l}\text { B. Burns, M.G. Wellls \#1 (dry hole) (Young County field) } \\
\text { TE\&L Co. Sur. Blk 206, Abst. } 453,330 \mathrm{ft} \text { E \& } 330 \mathrm{ft} \text { S of NW Cor. of S } 1 / 2 \text { TE\&L Co. Suv. \#206, } \\
4.5 \mathrm{mi} \text { SE fr Olney }\end{array}$ & & 5006 & 9/4/1946 & 9/30/1946 & $\begin{array}{c}\text { coal: } 241 \\
\text { coal: } 370 \\
\text { coal: } 391 \\
\text { coal: } 1317 \\
\end{array}$ \\
\hline 460 & $\begin{array}{l}\text { B. Burns, D. Wilborn \#3 (dry hole) (Young County field) } \\
\text { TE\&\&L Co. Sur. Abst. 930, Sec. 1588, } 320 \text { Ac., } 150 \text { ft FWL \& } 750 \text { ft FNL, } 2 \text { mi NE of Spring Creek }\end{array}$ & & 1371 & 6/29/1955 & 6/30/1955 & coal: 1009 \\
\hline 461 & $\begin{array}{l}\text { B. Burns L.T. Burns Operator, W. T. Rogers \#1 (oil prod) (N Young County field) } \\
\text { TE\&L A-466, BIk } 219,150 \mathrm{ft} S \& \text { E of NW Cor. E } 40 \text { ac of W } 80 \text { S } 1 / 2 \text { Suv. A-466, Blk } 219 \\
\text { (Gunsite lime at } 855 \mathrm{ft} \text { ) }\end{array}$ & & 877 & 10/2/1940 & $10 / 7 / 1940$ & coal: 482 \\
\hline 462 & $\begin{array}{l}\text { B. Burn L.T. Burns Operator, W. T. Rogers } \# 2 \text { (oil prod) (N Young County field) } \\
\text { TE\&L Suv. A-466, Blk } 219,150 \mathrm{ft} \text { \& } 450 \mathrm{ft} \text { of NWW Cor. E } 40 \text { ac of W } 80 \text { S 1/2 Suv. A-466, Blk } \\
219 \text { (Gunsite lime at } 855 \mathrm{ft} \text { ) }\end{array}$ & & 880 & 10/8/1940 & 10/8/1940 & coal: 477 \\
\hline 463 & $\begin{array}{l}\text { B. Burns L.T. Burns Operator, W. T. Rogers \#3 (oil prod) (N Young County field) } \\
\text { TE\&L A-466, Blk } 219,450 \mathrm{ft} S \& \mathrm{E} \text { of } \mathrm{NW} \text { Cor. Of E } 40 \text { ac of W } 80 \mathrm{~S} 1 / 2 \text { Suv. (Gunsite lime at } 855 \mathrm{ft} \text { ) }\end{array}$ & & 880 & $10 / 11 / 1940$ & 10/14/1940 & coal: 478 \\
\hline 464 & $\begin{array}{l}\text { L.T. Burns Operator for B. Burns, W. T. Rogers \#4 (dry hole) (N Young County field) } \\
\text { TE\&L Co. Suv., Bk } 219 \text {, Abst. } 466,450 \mathrm{ft} \text { \& } 150 \mathrm{ft} \text { of NW Cor. Of E-40 of W } 80 \text { Ac. of } \\
\text { S1/2 Suv. \#219 (lime G.S. at } 857 \mathrm{ft} \text { ) }\end{array}$ & & 919 & 12/28/1941 & $12 / 30 / 1941$ & Sh, blk: 866 \\
\hline 465 & $\begin{array}{l}\text { B. Burns L.T. Burns Operator, W. T. Rogers \#5 (oil prod) (N Young County field) } \\
\text { TE\&L A-466, Blk } 219,750 \mathrm{ft} E \& 150 \mathrm{ft} \text { S of NW Cor. of E } 40 \text { of W } 80 \text { ac S } 1 / 2 \text { Sur. A-466, 300m ft E } \\
\text { of Well \#2 (Gunsite lime at } 843 \mathrm{ft} \text { ) }\end{array}$ & & 882 & $\begin{array}{l}10 / 14 / 1940 \\
\end{array}$ & 19/17/1940 & $\begin{array}{l}\text { Sh \& coal: } 455 \\
\text { coal: } 479\end{array}$ \\
\hline 466 & $\begin{array}{l}\text { B. Burns L.T. Burns Operator, W. T. Rogers \#6 (oil prod) (N Young County field) } \\
\text { TE\&L A-466, Blk } 219,750 \mathrm{ft} E \& 450 \mathrm{ft} \text { Sof NW Cor. of E } 40 \text { of W } 80 \text { of S } 1 / 2 \text { Suv. A-466, Blk 219, } \\
300 \mathrm{ft} S \text { of \#5 (Gunsite lime at } 842 \mathrm{ft} \text { ) }\end{array}$ & & 890 & 10/17/1940 & 10/20/1940 & coal: 476 \\
\hline 467 & $\begin{array}{l}\text { L.T. Burns, S.J. Allison \#2 (dry hole) (Young County field) } \\
\text { TE\&L Co. Suv., Blk } 150 \text {, Abst. } 397,40 \text { Ac., } 1134.2 \mathrm{ft} \text { W \& } 938.2 \mathrm{ft} \text { S of NW Cor. Suv. \#150, } 330 \mathrm{ft} N \text { \& } \\
\text { W of SE Cor. } 40 \text { Ac Lse., } 1.5 \mathrm{mi} \mathrm{N} \text { of Orth }\end{array}$ & & 3347 & $1 / 5 / 1945$ & 1/28/1945 & $\begin{array}{c}\text { coal: } 460 \\
\text { S, Sh, coal stk: } 909\end{array}$ \\
\hline 468 & $\begin{array}{l}\text { L.T. Burns, Trustee, R.O. Andrews \#3 (oil prod) } \\
\text { TE\&L Co. A-477, } 300 \mathrm{ftt} \text { of Well } \# 2 \\
\text { (lime G.S. at } 844 \mathrm{ft} \text { ) }\end{array}$ & $1166 \mathrm{GL}$ & 920 & $1 / 25 / 1936$ & $2 / 6 / 1936$ & $\begin{array}{l}\text { coal: } 508 \\
\text { Sh, gy, dark: } 540 \\
\text { Sh, blk: } 840 \\
\end{array}$ \\
\hline 469 & $\begin{array}{l}\text { L.T. Burns, Trustee, R.O. Andrews \#4 (oil prod) } \\
\text { TE\&LL Co. Suv. Blk } 1399 \text {, Abst. } 477,300 \mathrm{ft} \text { E of Well \#3, or } 586.6 \mathrm{fW} \& 150 \mathrm{ft} \mathrm{N} \text { of SE Cor. of lease } \\
\text { (lime G.S. at } 842 \mathrm{ft} \text { ) }\end{array}$ & & 918 & $2 / 8 / 1936$ & $2 / 21 / 1936$ & $\begin{array}{l}\text { Sh, blk: } 515 \\
\text { Sh, blk: } 839\end{array}$ \\
\hline 470 & $\begin{array}{l}\text { L.T. Burns, Trustee, R.0. Andrews \#5 (oil prod) } \\
\text { TE\&L Co. Suv. Blk } 1399, \text { Abst. } 477,150 \mathrm{ftE} \text { \& } 450 \mathrm{ft} \mathrm{N} \text { of SW Cor. of Ise or } 300 \mathrm{ft} \mathrm{N} \text { of Well \#2 } \\
\text { (lime GS at } 852 \mathrm{ft} \text { ) }\end{array}$ & & & & & $\begin{array}{l}\text { Sh, blk: } 518 \\
\text { Sh, blk: } 848\end{array}$ \\
\hline 471 & $\begin{array}{l}\text { L.T. Burns, Trustee, R.0. Andrews \#7 (dry hole) } \\
\text { TE\&L Co. Suv. Blk 1399, Abst. } 477,315 \mathrm{ft} N \text { \& } 115 \mathrm{ft} \text { E of Well \#4, or } 471.6 \mathrm{ft} \text { W \& } 465 \mathrm{ft} \mathrm{N} \text { of SE Cor. }\end{array}$ & & 951 & 11/2/1936 & 11/20/936 & $\begin{array}{l}\text { Sh, blk: } 250 \\
\text { Sh, blk: } 435 \\
\text { Sh, blk: } 517 \\
\text { Sh, dark: } 705 \\
\text { Sh. blk: } 841\end{array}$ \\
\hline 472 & $\begin{array}{l}\text { L.T. Burns, W.S. Bailey Jr. \#1 (dry hole) (Young County field) } \\
\text { TE\&L Co. Suv. Blk 60, Sec. A-336, } 150 \mathrm{ft} N \& \text { W of SE Cor. of W } 1 / 2 \text { of TE\&L Co. Suv. \#60, A-336, } \\
0.5 \mathrm{mi} W \text { fr Oth }\end{array}$ & & 875 & 2/10/1941 & 2/12/1941 & $\begin{array}{l}\text { Sh \& coal: } 445 \\
\text { coal: } 520 \\
\text { Sh, blk: } 610\end{array}$ \\
\hline 473 & $\begin{array}{l}\text { L.T. Burns, S.M.J.J. Benson Estate \#1 (dry hole) } \\
\text { TE\&L Co. Suv. Blk 1478, Abst. } 904,1500 \mathrm{ft} N \& 470 \mathrm{ft} \text { W of SE Cor. of } 132 \text { Ac. Lse., being E-132 } \\
\text { Ac. of Suv. }\end{array}$ & & 956 & 3/13/1936 & 3/26/1936 & $\begin{array}{l}\text { Sh, blik: } 525 \\
\text { Sh, blk: } 224\end{array}$ \\
\hline 474 & $\begin{array}{l}\text { L.T. Burns, S.M.J. Benson Estate \#C-1 (dry hole) } \\
\text { TE\&\&L Co. Suv. \#1482, } 800 \mathrm{ft} \text { N \& } 800 \mathrm{ft} \text { S of NE Corner }\end{array}$ & & 952 & $5 / 10 / 1936$ & $5 / 24 / 1936$ & $\begin{array}{l}\text { coal: } 510 \\
\text { Sh, blk: } 864\end{array}$ \\
\hline 475 & $\begin{array}{l}\text { L.T. Burns, J.E. Bishop \#1 (dry hole) } \\
\text { TE\&L Co. Suv. Blk } 162 \text {, Abst. } 409,80 \text { Ac., } 330 \mathrm{ft} \text { each way out of SW Cor. Of N } 1 / 2 \text { of TE\&L Suv. \#162 }\end{array}$ & & 4957 & $12 / 20 / 1945$ & $2 / 8 / 1946$ & $\begin{array}{l}\text { coal: } 540 \\
\text { coal: } 565\end{array}$ \\
\hline 476 & $\begin{array}{l}\text { L.T. Burns B.M. Burns, J.E. Bishop \#2 (oil prod) (Crain-Kunkel field) } \\
\text { TE\&L Co. Suv. Blk 162, Sec. A-409, } 80 \text { Ac., } 330 \mathrm{ft} \text { FWL \& } 660 \mathrm{ft} \text { FSL of N/2 of Surv \#162 }\end{array}$ & & 4912 & $8 / 17 / 1953$ & 9/10/1953 & $\begin{array}{c}\text { coal: } 528 \\
\text { coal: } 1533\end{array}$ \\
\hline 477 & $\begin{array}{l}\text { L.T. Burns, J.M. Bloodworth \#1 (dry hole) (Young County field) } \\
\text { TE\&L Co. Suv. Blk } 210, \text { Abst. } 457,1977 \mathrm{ft} W \& 150 \mathrm{ft} \text { Nof SE Cor. Of Bloodworth Ld out Suv. \#210 }\end{array}$ & & 883 & 11/1/1940 & 11//4/1940 & coal: 464 \\
\hline 478 & $\begin{array}{l}\text { L.T. Burns, H.E. Bloodworth \#1 (dry hole) (Young County field) } \\
\text { TE\&L Co. A-461, Blk 214, } 783 \mathrm{ft} \text { \& \& } 150 \mathrm{ft} \text { of of NW Cor. Blk 24, Suv. A-461 }\end{array}$ & & 903 & $2 / 16 / 1941$ & $2 / 18 / 1941$ & $\begin{array}{l}\text { Sh \& coal: } 445 \\
\text { Sh \& coal: } 485 \\
\text { Sh \& coal: } 504\end{array}$ \\
\hline 479 & $\begin{array}{l}\text { L.T. Burns, H.E. Bloodworth \#2 (dry hole) (Young County field) } \\
\text { TE\&L Co. A-464, Blk } 217,450 \mathrm{ft} W \& 150 \mathrm{ft} \text { Nof SE Cor. of N } 20 \text { of E } 40 \text { of SW } 80 \text { Ac. Suv. } 217 \text { or } \\
1416 \mathrm{ft} E \& 1083 \mathrm{ft} \text { Nof SW Cor. suv. (Gunsight lime @ } 890.5 \mathrm{ft} \text { ) }\end{array}$ & & 952 & $5 / 4 / 1941$ & $5 / 6 / 1941$ & $\begin{array}{l}\text { Sh \& coal: } 467 \\
\text { Sh, blk: } 890\end{array}$ \\
\hline 480 & $\begin{array}{l}\text { L.T. Burns, J.M. Bloodworth (Shell) \#2 (loil prod) (Young County field) } \\
\text { TE\&LL. Co. Suv. Blk } 210,1330 \mathrm{ft} \mathrm{W} \& 750 \mathrm{ftS} \mathrm{of} \mathrm{NE} \mathrm{Cor.} \mathrm{Bloodworth} \mathrm{Land} \mathrm{out} \mathrm{Suv.} \mathrm{\# 210} \mathrm{or} 1330 \mathrm{ft} \text { W \& } \\
1332 \mathrm{ft} \text { Sof NE Cor. of Suv. (G.S. lime @ } 834 \mathrm{ft} \text { ) }\end{array}$ & & 884 & 6/11/1941 & 6/14/1941 & Sh \& coal: 475 \\
\hline 481 & $\begin{array}{l}\text { L.T. Burns, J.M. Bloodworth \#3 (oil prod) (Young County field) } \\
\text { TE\&L Co. Suv. Blk 210, A-457, } 1330 \mathrm{ft} \text { W \& } 450 \mathrm{ft} \text { S of NE Cor. }\end{array}$ & & 885 & 1/16/1941 & 1/21/1941 & coal \& Sh: 407 \\
\hline 482 & $\begin{array}{l}\text { L.T. Burns, J.M. Bloodworth (Shell) \#4 (dry hole) (Young County field) } \\
\text { TE\&L Co. Suv. Blk 210, A-457, } 1330 \mathrm{ft} \text { W \& } 1050 \mathrm{ft} \text { S of NE Cor. TE\&L Suv. A-457, Blk } 210\end{array}$ & & 929 & 1/13/1941 & 1/15/1941 & $\begin{array}{c}\text { coal: } 405 \\
\text { coal: } 471 \\
\text { Sh \& coal: } 580\end{array}$ \\
\hline 483 & $\begin{array}{l}\text { L.T. Burns, J.M. Bloodworth-Shell) \#5 (oil prod) (Young County field) } \\
\text { TE\&\&L Co. Suv. Blk 210, A-457, } 1650 \mathrm{ft} W \& 450 \mathrm{ft} \text { of NE Cor. of Bloodworth land out Suv. Blk } 210\end{array}$ & & 885 & 1/21/1941 & 1/25/1941 & coal: 417 \\
\hline 484 & $\begin{array}{l}\text { L.T. Burns, J.M. Bloodworth-Shell \#7 (oil prod) (Young County field) } \\
\text { TE\&\&L Suv. BIk 210, A-457, } 1630 \mathrm{ft} \text { W \& } 150 \mathrm{ft} \text { S of NE Cor. Suv. A-467, BIk } 210\end{array}$ & & 887 & 1/28/1941 & $2 / 5 / 1941$ & $\begin{array}{l}\text { coal \& Sh: } 401 \\
\text { coal: } 445\end{array}$ \\
\hline 485 & $\begin{array}{l}\text { L.T. Burns, J.M. Bloodworth \#9 (oil prod) (Young County field) } \\
\text { TE\&L Co. Suv. Blk 210, Ab. } 457,1930 \mathrm{ft} W \& 450 \mathrm{ft} \text { S of NE Cor. Bloodworth Ld. out of Suv. \#210. } \\
300 \mathrm{ft} \text { S of } \# 8 \text { (G.S. lime @ } 834 \mathrm{ft} \text { ) }\end{array}$ & & 884 & 6/20/1941 & 6/23/1941 & $\begin{array}{c}\text { coal: } 465 \\
\text { Sh, blk: } 832\end{array}$ \\
\hline 486 & $\begin{array}{l}\text { L.T. Burns, J.M. Bloodworth \#1 10 (oil prod) (Young County field) } \\
\text { TE\&L Co. Suv. Blk } 210,1630 \mathrm{ft} \text { \& } 1332 \mathrm{ft} \text { of of NE Cor. Bloodworth Ld. (G.S. lime @ } 832 \mathrm{ft} \text { ) }\end{array}$ & & 883 & 6/17/1941 & $6 / 20 / 1941$ & $\begin{array}{l}\text { Sh \& coal: } 414 \\
\text { coal: } 466 \\
\text { Sh, blk: } 831\end{array}$ \\
\hline 487 & $\begin{array}{l}\text { L.T. Burns, J.M. Bloodworth \#11 (oil prod)(Young County field) } \\
\text { TE\&L Co. Suv. Blk 210, } 1330 \mathrm{ft} \text { W \& } 750 \mathrm{ft} \text { S of NE Cor. of Bloodworth Ld. Out of TE\&L Suv. \#210 } \\
\text { (G.S. lime @ } 831 \mathrm{ftt} \text { ) }\end{array}$ & & 886 & 6/3/1941 & $6 / 5 / 1941$ & $\begin{array}{l}\text { Coual: } 480 \\
\text { Sh, blk: } 830\end{array}$ \\
\hline 488 & $\begin{array}{l}\text { L.T. Burns, H. Braune \#1 (dry hole) } \\
\text { TE\&L Co. Suv. Blk } 188,150 \mathrm{ft} N \& 450 \mathrm{ft} \text { W of SE Cor. of NW } 1 / 4 \text { of Suv. } 188\end{array}$ & & 1259 & $5 / 2 / 1936$ & $5 / 6 / 1936$ & $\begin{array}{l}\text { coal: } 485 \\
\text { Sh, blk: } 830 \\
\text { Sh, blk: } 1167\end{array}$ \\
\hline 489 & $\begin{array}{l}\text { L.T. Burns, N. Brooks \#1 (oil prod) (Williamson field) } \\
\text { TE\&\&L Co. Suv. \#299, Abst. 546, } 467 \mathrm{ft} \text { each way out of SW Cor. of TE\&L Co. Suv. \#299 }\end{array}$ & & 4952 & 6/22/1944 & 7/21/1944 & coal: 117 \\
\hline 490 & $\begin{array}{l}\text { L.T. Burns, N. Brooks \#3 (dry hole) (Young County field) } \\
\text { TE\&\&L Co. Suv. Blk 299, Abst. } 546,492 \mathrm{ft} N \& 492 \mathrm{ft} \text { of SW Cor. Suv., } 1.75 \mathrm{mi} \text { SW fr Jean }\end{array}$ & & 1420 & 10/2/1946 & 10/11/1946 & $\begin{array}{l}\text { Sh \& coal: } 704 \\
\text { Sh, dark: } 1403\end{array}$ \\
\hline 491 & $\begin{array}{l}\text { L.T. Burns, N. Brooks (Crain) \#1 (oil prod) (WC) } \\
\text { TE\&L Co. Suv. Blk } 169 \text {, Abst. 416, } 330 \mathrm{ft} \text { N \& W of SE Cor. of N1/2 of Suv. 169, 416, being } 1537 \mathrm{ft} \mathrm{S} \mathrm{\&} \\
330 \mathrm{ft} \text { W of NE Corner f Suv. }\end{array}$ & & 4957 & 9/4/1945 & $10 / 13 / 1945$ & $\begin{array}{c}\text { coal: } 526 \\
\text { coal: } 646 \\
\text { coal: } 989 \\
\text { coal: } 1006 \\
\text { coal: } 1555\end{array}$ \\
\hline
\end{tabular}




\begin{tabular}{|c|c|c|c|c|c|c|}
\hline $\begin{array}{c}\text { Occurrence } \\
\text { No. }\end{array}$ & $\begin{array}{l}\text { Well name / } \\
\text { location }\end{array}$ & $\begin{array}{c}\text { Elevation } \\
\text { (ft) }\end{array}$ & $\begin{array}{c}\text { Total } \\
\text { depth (ft) }\end{array}$ & $\begin{array}{l}\text { Spud } \\
\text { date }\end{array}$ & $\begin{array}{c}\text { Compl; PB } \\
\text { dates }\end{array}$ & $\begin{array}{l}\text { Coal and black or dark-gray shale } \\
\text { occurrences (depth in ft) }\end{array}$ \\
\hline 492 & $\begin{array}{l}\text { L.T. Burns, Trustee, E. Burris \#1 (dry hole) (WC) } \\
\text { TE\&\&L Co. Suv. Blk 205, Abst. } 452,240 \text { Ac., } 660 \text { ft N \& } 330 \mathrm{ft} \text { W of SE Cor. Suv. 205, } 2.75 \text { mi NE fr True }\end{array}$ & & 5035 & $5 / 7 / 1944$ & 6/15/1944 & $\begin{array}{c}\text { coal: } 249 \\
\text { coal: } 344 \\
\text { Sh, blk: } 1236\end{array}$ \\
\hline 493 & $\begin{array}{l}\text { L.T. Burns, R. Campbell Est. \#1-B (dry hole) (Young County field) } \\
\text { TE\&\&L Co. Suv. Blk } 1383,80 \text { Ac., } 450 \mathrm{ft} N \text { \& } 750 \mathrm{ft} \text { Wof SE Cor. S1/2 Suv. \#1383 }\end{array}$ & & 1074 & $10 / 21 / 1945$ & $10 / 23 / 1945$ & $\begin{array}{l}\text { Sh, sdy, coal strk: } 608 \\
\text { Sh \& coal: } 653 \\
\text { coal: } 982\end{array}$ \\
\hline 494 & $\begin{array}{l}\text { L.T. Burns, R. Campbell } \# 2 \text { (oil prod) } \\
\text { TE\&L Co. Suv. Blk } 1484,450 \mathrm{ft} S \& 500 \mathrm{ft} W \text { of NE Cor. of lease or } 350 \mathrm{ft} W \text { of } \# 1\end{array}$ & & 920 & 3/8/1936 & 3/24/1936 & Sh, blk: 911 \\
\hline 495 & $\begin{array}{l}\text { L.T. Burns, R. Campbell Est."C" \#1 (oil prod) (Young County field) } \\
\text { TE\&L Co. Suv. Blk 1376, Abst. 264, } 1300 \mathrm{ft} \text { \& } 467 \mathrm{ft} \text { S of NW Cor. of Suv. } 1376\end{array}$ & & 4605 & 5/15/1947 & 6/15/1947 & coal: 276 \\
\hline 496 & $\begin{array}{l}\text { L.T. Burns, B. Campbell Est."E" \#1 (oil prod) (Young County Regular field) } \\
\text { TE\&L Co. Suv. Blk } 1452,430 \text { Ac.,150 ft E \& } 450 \mathrm{ft} \text { S of NW Cor. of Blk } 1452\end{array}$ & & 899 & $6 / 8 / 1948$ & 6/9/1949 & coal: 488 \\
\hline 497 & $\begin{array}{l}\text { L.T. Burns, R. Campbell Est."E" \#2 (oil prod)( (Young County Regular field) } \\
\text { TE\&L Co. Suv. Blk 1452, Abst. } 878,430 \text { Ac..450 ft S \& } 450 \mathrm{ft} \text {. of NWW Cor. of Suv. } 1452,300 \mathrm{ft} \text {. of \#1 }\end{array}$ & & 953 & 6/11/1948 & 6/12/1948 & $\begin{array}{c}\text { coal: } 488 \\
\text { Sh \& coal: } 553\end{array}$ \\
\hline 498 & $\begin{array}{l}\text { L.T. Burns, R. Campbell Est."E" \#3 (oil prod) (Young County Regular field) } \\
\text { TE\&L Co. Suv. Blk 1452, } 430 \text { Ac., } 750 \mathrm{ft} S \& 150 \mathrm{ft} \text { E of NW Cor. of Suv. \#1452, } 300 \mathrm{ft} \mathrm{S} \text { of \#1 }\end{array}$ & & 898 & 6/15/1948 & 6/16/1948 & coal: 479 \\
\hline 499 & $\begin{array}{l}\text { L.T. Burns, R. Campbell Est."E" \#4 (oil prod) (Young County Regular field) } \\
\text { TE\&L Co. Suv. Blk } 1452,430 \text { Ac., } 1050 \mathrm{ft} S \& 150 \mathrm{ft} \text { E of NW Cor. of TE\&L Suv. \#1452, } 300 \mathrm{ft} \text { S of \#3 }\end{array}$ & & 898 & $6 / 18 / 1948$ & $6 / 20 / 1948$ & coal: 477 \\
\hline 500 & $\begin{array}{l}\text { L.T. Burns, R. Campbell Est."E" \#5 (oil prod) (Young County Regular field) } \\
\text { TE\&L Co. Suv. Blk 1452, Ab. } 878,430 \text { Ac., } 1350 \mathrm{ft} S \& 150 \mathrm{ft} \text { Eof NW Cor. of TE\&L Suv. \#1452, } \\
300 \mathrm{ft} \text { S of \#4 }\end{array}$ & & 8894 & 6/21/1948 & 6/22/1948 & $\begin{array}{c}\text { coal: } 472 \\
\text { Sh, blk: } 888\end{array}$ \\
\hline 501 & $\begin{array}{l}\text { L.T. Burns, R. Campbell Est."E" \#6 (oil prod)(Young field); TE\&L Co. Suv. } \\
\text { BIk 1452, Ab. } 878,430 \text { Ac., } 1650 \mathrm{ft} \text { \& } 150 \mathrm{ft} \text { of NW Cor. Suv. } 1452,5.5 \mathrm{mi} \text { Efr Olney }\end{array}$ & & 1108 & 6/26/1948 & 6/29/1948 & Sh, blk: 901 \\
\hline 502 & 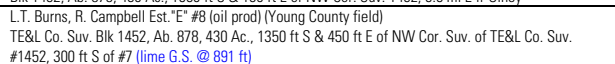 & & 900 & $7 / 8 / 1948$ & $7 / 9 / 1948$ & $\begin{array}{l}\text { Sh \& coal stks: } 875 \\
\text { Sh, blk: } 887\end{array}$ \\
\hline 503 & $\begin{array}{l}\text { L.T. Burns, R. Campbell Est."E" \#10 (dry hole) (Young County field) } \\
\text { TE\&LL Co. Suv. Blk 1452, Ab. } 878,430 \text { Ac., } 900 \mathrm{ft} \text { \& } 750 \mathrm{ft} \text { of NW Cor. of Suv. \#1452, } \\
5.5 \mathrm{mi} \text { E fr Olney (lime G.S. @ } 902 \mathrm{ft} \text { ) }\end{array}$ & & 950 & $7 / 13 / 1948$ & $7 / 16 / 1948$ & Sh, blk: 891 \\
\hline 504 & $\begin{array}{l}\text { L.T. Burns, R. Campbell Est."E" \#11 (oil prod) (Young County field) } \\
\text { TE\&L Co. Suv. Blk 1452, Ab. } 878,1200 \mathrm{ft} \text { \& } 750 \mathrm{ft} \text { of NW Cor. of TE\&L Co. Suv. } 1452\end{array}$ & & 910 & 7/22/1948 & 7/23/1948 & $\begin{array}{c}\text { coal: } 471 \\
\text { Sh, blk: } 896\end{array}$ \\
\hline 505 & $\begin{array}{l}\text { L.T. Burns, , C. Campbell Est."E" \#13 (oil prod) (Young County field) } \\
\text { TE\&L Co. Suv. Blk 1452, Ab. } 878,1500 \mathrm{ft} S \& 750 \mathrm{ftE} \text { of NW Cor. Suv. \#1452,300 ft S of \#11 }\end{array}$ & & 908 & 7/26/1948 & 7/27/1948 & Sh, blk: 900 \\
\hline 506 & 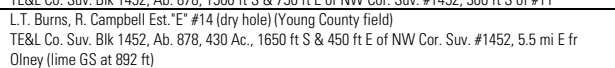 & & 950 & 7/30/1948 & 7/31/1948 & $\begin{array}{c}\text { coal: } 470 \\
\text { Sh, blk: } 890\end{array}$ \\
\hline 507 & $\begin{array}{l}\text { L.T. Burns, R. Campbell Est."E" \#17 (dry hole) (Young County field) } \\
\text { TE\&L Co. Suv. Blk } 1452 \text {, Sec.A-878, } 530 \text { Ac., } 330 \text { ft FEL \& } 1950 \mathrm{ft} \mathrm{FNL,} 6 \text { mi E fr Olney }\end{array}$ & & 984 & $3 / 8 / 1950$ & 3/10/1950 & Sh, blk: 912 \\
\hline 508 & $\begin{array}{l}\text { L.T. Burns, R. Campbell Est."E" \#18 (dry hole) (Young County field) } \\
\text { TE\&L Co. Suv. Blk } 1452 \text {, Sec.A-878, } 530 \text { Ac., } 1350 \mathrm{ft} \text { FNL \& } 330 \mathrm{ft} \text { FEL of Sec A-878, } 6 \text { mi E fr Olney }\end{array}$ & & 950 & $3 / 10 / 1950$ & $3 / 11 / 1950$ & Sh, blk: 906 \\
\hline 509 & $\begin{array}{l}\text { L.T. Burns, J.D. Carpenter \#9 (dry hole) } \\
\text { TE\&L Co. Suv. Blk } 1452 \text {, Abst. } 411 \text {, Sec. } 164,50 \mathrm{ft} \mathrm{N} \& 50 \mathrm{ft} \text { E of SW Cor. E } 40 \text { Ac. of N } 80 \text { Ac. } \\
\text { W1/2 of Suv. } 164\end{array}$ & & 935 & 7/18/1936 & 7/27/1936 & $\begin{array}{l}\text { Sh, blk: } 450 \\
\text { Sh, blk: } 465 \\
\text { Sh, blk: } 540 \\
\text { Sh, blk: } 875\end{array}$ \\
\hline 510 & $\begin{array}{l}\text { L.T. Burns, M.L. Christian \#1 (oil prod) } \\
\text { TE\&L Co. \#255-256, Abst. 502-503, } 1500 \mathrm{ft} \text { S of NE Cor. of Suv. 255, Abst. } 502 \& \text { being in the EL } \\
\text { of Sur. } 255 \& \text { WL of } 256\end{array}$ & 1122 & 4984 & 2/26/1944 & 4/6/1944 & Sh, blk: 1154 \\
\hline 511 & $\begin{array}{l}\text { L.T. Burns, M.L. Christian \#2 (dry hole) (WC) } \\
\text { TE\&L Co. \#255, Abst. } 502,394 \text { Ac., } 920 \mathrm{ft} \& \& 314 \mathrm{ft} \text { W of NE Cor. of Suv. \#255, } 660 \mathrm{ft} \text { NW of \#1 }\end{array}$ & & 2910 & 9/26/1944 & $\begin{array}{l}10 / 9 / 1944 \\
\end{array}$ & Sh, blk: 1167 \\
\hline 512 & $\begin{array}{l}\text { L.T. Burns, MLL. Christian \#3 (oil prod) (WC) } \\
\text { TE\&L Co. } 255-56,2160 \text { ft S of NE Cor. of TE\&L Co. Suv. 255, Abst. } 502 \text { \& being in EL of Suv. } 255 \text { \& } \\
\text { the WL of Suv. } 256\end{array}$ & & 2603 & 10/13/1944 & 10/23/1944 & Sh, blk: 1147 \\
\hline 513 & $\begin{array}{l}\text { L.T. Burns, M.L. Christian \#4 (oil prod) (Burns-Ragland: Strawn field) } \\
\text { TE\&L Co. Suv. Blk 256, Ab. } 503,1807 \mathrm{ft} S \& 572 \mathrm{ft} \text { of NW Cor. of Suv. \#256, } 660 \mathrm{ft} \text { SE of } \# 1,660 \mathrm{ft} \\
\text { NE of \#3 }\end{array}$ & & 2603 & 10/31//1944 & 11/15/1944 & $\begin{array}{l}\text { coal: } 292 \\
\text { caal: } 444 \\
\text { Sh, blk, hd: } 780 \\
\text { Sh, blk: } 1145\end{array}$ \\
\hline 514 & $\begin{array}{l}\text { L.T. Burns, M.L. Christian \#5 (oil prod) (WC) } \\
\text { TE\&L Co. Suv. Blk } 255 \text {, Ab. } 502,1650 \mathrm{ft} \text { \& } 660 \mathrm{ft} \text { W of NE Cor. of Suv. \#255, Abst. } 502 \& 150 \mathrm{ft} \mathrm{S} \& \\
660 \mathrm{ft} \text { W of \#1 well }\end{array}$ & & 2623 & 11/10/1944 & 11/30/1944 & $\begin{array}{c}\text { coal: } 773 \\
\text { Sh, blk: } 1155\end{array}$ \\
\hline 515 & $\begin{array}{l}\text { L.T. Burns, R. Clarke \#1 (dry hole) (Young County field) } \\
\text { TE\&\&L Co. Suv. 212, Abst. } 459,400 \mathrm{ft} N \& \text { E of SW Cor. N 1/2 of TE\&L Co. Suv. 212, Abst. } 459\end{array}$ & & 952 & $7 / 9 / 1941$ & 7/10/1941 & $\begin{array}{c}\text { coal: } 460 \\
\text { Sh, blk: } 867\end{array}$ \\
\hline 516 & $\begin{array}{l}\text { L.T. Burns, J.P. Dunagan \#1 (oil prod) (Young County field) } \\
\text { TE\&L Co. Suv. Blk 170, Abst. 417, } 330 \mathrm{ft} \text { \& } 330 \mathrm{ft} \text { of NE Cor. of TE\&L Co. Suv. 170, Abst. } 417\end{array}$ & & 5240 & 12/13/1945 & $1 / 18 / 1946$ & Sh, blk: 1591 \\
\hline 517 & $\begin{array}{l}\text { L.T. Burns, J.P. Dunagan "C" \#1 (dry hole) (WC) } \\
\text { TE\&LL Co. Suv. Blk 412, Ab. } 619,160 \text { Ac., } 330 \mathrm{ft} \text { FNL \& } 1263 \mathrm{ft} \text { FEL of Lse, } 6 \mathrm{mi} \text { SE fr Olney }\end{array}$ & & 5119 & 3/7/1951 & $3 / 21 / 1951$ & coal: 582 \\
\hline 518 & $\begin{array}{l}\text { L.T. Burns, C. Ellis \#1 (oil prod) (Davis field) } \\
\text { TE\&L Co. Suv. Blk 207. Ab. } 454,1400 \mathrm{ftE} \text { \& } 330 \mathrm{ft} S \text { of NW Cor. S1/2 Suv. \#207 }\end{array}$ & & 4865 & 10/4/1946 & 11/2/1946 & coal: 420 \\
\hline 519 & $\begin{array}{l}\text { L.T. Burns, C. Ellis \#4 (dry hole) (Young field) } \\
\text { TE\&L Co. Suv. Blk } 207, \text { Ab. \#454, } 160 \text { Ac., } 2000 \mathrm{ft} \text { E \& } 450 \mathrm{ft} \text { S of NW Cor. S 1/2 Suv. \#207 }\end{array}$ & & 775 & 10/23/1946 & 10/24/1946 & Sh, blk: 750 \\
\hline 520 & $\begin{array}{l}\text { L.T. Burns, E.J. Erreest \#1 (fry hole) (Young County field) } \\
\text { TE\&\&L Co. Sur. Blk } 64, \text { Ab. \#340, } 300 \text { Ac., } 450 \mathrm{ft} F W L \text { \& } 1650 \mathrm{ft} \text { FSL of TE\&L Suv. \#65 } \\
\text { (G.S. lime at } 905 \mathrm{ft} \text { ) }\end{array}$ & & 1120 & 8/13/1946 & 8/16/1946 & $\begin{array}{l}\text { Sh \& coal stk: } 632 \\
\text { Sh, blk: } 994\end{array}$ \\
\hline 521 & $\begin{array}{l}\text { L.T. Burns, S.J. Furr \#1 (dry hole) } \\
\text { TE\&L Co. Suv. Blk } 3 \text {, Abst. } 313,1080 \mathrm{ft} \text { \& \& } 1466 \mathrm{ft} \text { W of NE Cor. of S } 200 \text { Ac. } \\
\text { (lime Gunsight at } 702 \mathrm{ft} \text { ) }\end{array}$ & 1137 & 788 & $3 / 18 / 1935$ & $3 / 27 / 1935$ & $\begin{array}{c}\text { coal: } 193 \\
\text { caal: } 375 \\
\text { Sh. blk: } 698\end{array}$ \\
\hline 522 & 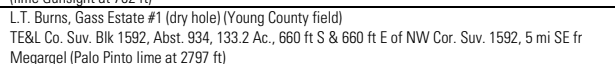 & & $1 / 7 / 1914$ & 8/7/1943 & 10/19/1943 & Chal: 840 \\
\hline 523 & 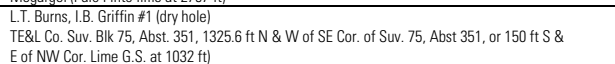 & 1232 DF & 1150 & 10/31/1935 & 11/6/1935 & Sh, blk: 751 \\
\hline 524 & $\begin{array}{l}\text { L.T. Burns, G.B. Hamilton Estate \#1-A (dry hole) } \\
\text { TE\&L Co. Suv. Blk } 1,1670 \mathrm{ft} W \& 942 \mathrm{ft} N \text { of SE Cor. of Suv. 1393. (lime GS at } 852 \mathrm{ft})\end{array}$ & & 974 & $5 / 27 / 1936$ & 6/9/1936 & $\begin{array}{l}\text { Sh, blk: } 510 \\
\text { Sh, dark: } 850\end{array}$ \\
\hline 525 & $\begin{array}{l}\text { L.T. Burns, G.B. Hamilton Est. \#B-1 (dry hole) } \\
\text { TE\&L Co. Suv. Blk 3401, } 557.5 \mathrm{ft} \text { W } 1182.7 \mathrm{ft} \mathrm{N} \text { of SE Cor. of } 163 \text { Ac. of Block } 3401\end{array}$ & & 997 & $2 / 7 / 1937$ & 2/15/1937 & $\begin{array}{l}\text { Sh, blk: } 430 \\
\text { Sh, blk: } 864\end{array}$ \\
\hline 526 & $\begin{array}{l}\text { L.T. Burns., C.E. Howard \#1 (dry hole) (Burns-Ragland fieldd) } \\
\text { TE\&\& Co. Suv. Blk 246, Abst. 493, } 80 \text { Ac., } 467 \mathrm{ft} N \& 467 \mathrm{ft} \text { W of NE Cor. of Suv. \#246, Abst, 493, } \\
4 \text { mi W fr Jean }\end{array}$ & & 4969 & 4/10/1946 & $5 / 20 / 1946$ & coal: 1218 \\
\hline 527 & $\begin{array}{l}\text { L.T. Burns, W.B. Howard \#1 (dry hole) } \\
\text { TE\&L. Co. Suv. Blk 1480, Abst. } 906,225 \text { Ac., } 1000 \mathrm{ft} E \& \text { \& } 750 \mathrm{ft} \text { S of NW Cor. of Suv. \#1480, Abst, } 906\end{array}$ & & 984 & 12/13/1938 & $12 / 21 / 1938$ & $\begin{array}{l}\text { Sh, blk: } 478 \\
\text { Sh, blk: } 545 \\
\text { Sh, blk: } 552 \\
\text { Sh, blk: } 891\end{array}$ \\
\hline 528 & $\begin{array}{l}\text { L.T. Burns, W.B. Howard \#2 (oil prod) (Young County field) } \\
\text { TE\&\&L Co. Suv. Blk } 1480, \text { Abst. 906, } 225 \text { Ac., } 600 \mathrm{ft} N \text { \& } 150 \mathrm{ft} \text { W of SE Cor. TE\&L Co. Suv. \#11480 }\end{array}$ & & 907 & 6/11/1946 & 6/13/1946 & Sh \& coal: 555 \\
\hline 529 & 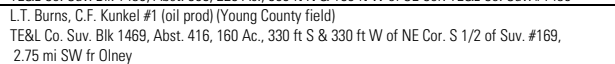 & & 4904 & 10/17/1945 & $11 / 10 / 1945$ & $\begin{array}{l}\text { coal: } 545 \\
\text { coal: } 575\end{array}$ \\
\hline 530 & $\begin{array}{l}\text { L.T. Burns, C.F. Kunkel \#1-A (oil prod) (Young County field) } \\
\text { TE\&L Co. Suv. Blk 1469, Abst. } 416,480 \mathrm{ft} S \& 330 \mathrm{ft} \text { W of NE Cor. S 1/2 of TE\&L Co. Suv. } 169 \text {, } \\
150 \mathrm{ft} \text { of Well \#1 }\end{array}$ & & 4898 & 11/24/1945 & $12 / 27 / 1945$ & coal: 545 \\
\hline 531 & $\begin{array}{l}\text { L.T. Burns, E.J. Larimore \#1 (oil prod) (Young County field); TE\&L Co. } \\
\text { Suv. A-457, Blk 210, } 2077 \mathrm{ft} \text { W } 150 \mathrm{ft} \text { S of NE Cor. A-457, Blk } 210 \text { (Gunsite lime at } 830.5 \mathrm{ft} \text { ) }\end{array}$ & & 876 & $10 / 28 / 1940$ & 10/31/1940 & coal: 476 \\
\hline 532 & 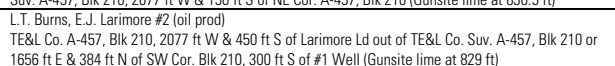 & & 878 & $11 / 5 / 1940$ & $11 / 7 / 1940$ & coal: 475 \\
\hline 533 & 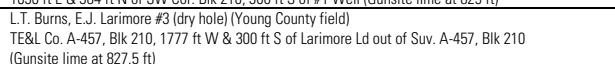 & & 900 & $1 / 1 / 1941$ & $1 / 3 / 1941$ & $\begin{array}{l}\text { coal: } 401 \\
\text { coal: } 467\end{array}$ \\
\hline 534 & $\begin{array}{l}\text { L.T. Burns, E.J.Larimore \#4 (oil prod) (Young County field) } \\
\text { TE\&L Co. A-457, Blk 210, } 2377 \mathrm{ft} \text { W \& } 450 \mathrm{ft} \text { Sof Larimore Ld out of TE\&L Suv. A-457, } 300 \mathrm{ft} \text { W of \#2 }\end{array}$ & & 878 & 1/6/1941 & 1/9/1941 & coal: 474 \\
\hline
\end{tabular}




\begin{tabular}{|c|c|c|c|c|c|c|}
\hline $\begin{array}{l}\text { Occurrence } \\
\text { No. }\end{array}$ & $\begin{array}{l}\text { Well name / } \\
\text { location }\end{array}$ & $\begin{array}{c}\text { Elevation } \\
(f t)\end{array}$ & $\begin{array}{c}\text { Total } \\
\text { depth (ft) }\end{array}$ & $\begin{array}{l}\text { Spud } \\
\text { date }\end{array}$ & $\begin{array}{c}\text { Compl; PB } \\
\text { dates }\end{array}$ & $\begin{array}{l}\text { Coal and black or dark-gray shale } \\
\text { occurrences (depth in ft) }\end{array}$ \\
\hline 535 & $\begin{array}{l}\text { L.T. Burns, E.J. Larimore \#5 (oil prod) (Young County field) } \\
\text { TE\&L Suv. A-457, Blk } 210,2377 \mathrm{ft} \text { of EL \& } 150 \mathrm{ft} \text { S of NL, A-457, Blk 2210, } 300 \mathrm{ft} W \text { of \#1 }\end{array}$ & & 879 & $1 / 9 / 1941$ & $1 / 12 / 1941$ & coal: 474 \\
\hline 536 & $\begin{array}{l}\text { L.T. Burns, E.J. Larimore \#6 (oil prod) (Young County field) } \\
\text { TE\&L Suv. A-457, } 2677 \mathrm{ft} \text { W \& } 450 \mathrm{ft} \text { S of Larimore Ld out TE\&L Co. Suv. } 210,300 \mathrm{ft} \text { W of Well \#4 } \\
\text { (Gunsight lime at } 342.5 \mathrm{ft} \text { ) }\end{array}$ & & 875 & 5/14/1941 & $5 / 26 / 1941$ & Sh \& coal: 475 \\
\hline 537 & $\begin{array}{l}\text { L.T. Burns, E.J. Larimore \#7 (dry hole) (Young County field) } \\
\text { TE\&L Co. Abst. } 457, \text { Blk } 210,2977 \mathrm{ft} \mathrm{W} \& 450 \mathrm{ft} \text { S of Larimore Ld out TE\&LL Co. Suv. \#210, } \\
300 \mathrm{ft} W \text { of Well } \# 6 \text { (G.S. lime at } 832 \mathrm{ft} \text { ) }\end{array}$ & & 887 & 7/15/1941 & 7/17/1941 & $\begin{array}{c}\text { coal: } 477 \\
\text { coal: } 571\end{array}$ \\
\hline 538 & $\begin{array}{l}\text { L.T. Burns, E.J. Larimore \#1-A (oil prod) (Young County field) } \\
\text { TE\&L Co. Suv. Blk } 150 \text {, Ab. } 397,500 \mathrm{ft} S \& \text { E of NW Cor. of E } 200 \text { Ac. Suv. 150, being } 1833 \mathrm{ft} \text { W \& } \\
500 \mathrm{ft} \text { S of NE Cor. of said Survey (limeGS at } 885 \mathrm{ft} \text { ) }\end{array}$ & & 4927 & 3/14/1941 & 4/12/1941 & coal: 1412 \\
\hline 539 & $\begin{array}{l}\text { L.T. Burns Estate, E.J. Larimore A AA-5 (dry hole) (Orth-Caddo field) } \\
\text { TE\&L Co. Suv. Abst. 397, Blk } 150,200 \text { ac., } 330 \mathrm{ft} \text { FSL \& } 330 \mathrm{ft} \text { FEL of Sur., } 0.75 \mathrm{mi} \mathrm{N} \text { fr Orth }\end{array}$ & & 4969 & 1/8/1955 & 1/25/1955 & coal: 248 \\
\hline 540 & $\begin{array}{l}\text { L.T. Burns, Larimore Estate \#2 (oil prod) (Young County field) } \\
\text { TE\&L Co. Suv. Blk 253, Sec. A-500, } 320 \text { acres, } 660 \mathrm{ft} \mathrm{N} \& 902 \mathrm{ft} \text { E of SW cor } 320 \text { acres }\end{array}$ & & 2872 & 2/11/1949 & 2/19/1949 & coal: 239 \\
\hline 541 & $\begin{array}{l}\text { L.T. Burns, Larimore Est. \#3 (dry hole) (Young County field) } \\
\text { TE\&L Co. Suv. Blk 253, Sec. A-500, } 320 \text { acres, } 400 \mathrm{ft} E \& 1252 \mathrm{ft} N \text { of SW cor of sur., } 1.5 \mathrm{mi} \text { SE fr True }\end{array}$ & & 2906 & 2/21/1949 & 2/28/1949 & $\begin{array}{c}\text { coal: } 237 \\
\text { coal: } 1140\end{array}$ \\
\hline 542 & $\begin{array}{l}\text { L.T. Burns, Larimore Est. \#4 (dry hole) (Young County field) } \\
\text { TE\&L Co. Sur. Blk 253, A-500, } 1474 \mathrm{ft} E \& 990 \mathrm{ft} \mathrm{N} \text { of SW cor., being } 660 \mathrm{ft} \mathrm{NE} \text { of } \# 2,1.5 \mathrm{mi} \text { SE fr True }\end{array}$ & & 2880 & $3 / 27 / 1949$ & 4/6/1949 & $\begin{array}{c}\text { coal: } 95 \\
\text { coal: } 252 \\
\text { Sh \& coal: } 470 \\
\text { coal: } 811 \\
\text { coal slate: } 1401\end{array}$ \\
\hline 543 & $\begin{array}{l}\text { L.T. Burns, C.D. Leberman \#1 (dry hole) (WC) } \\
\text { TE\&L Co. Sur. Blk 271, Abst } 518,320 \text { Ac., } 1650 \mathrm{ft} \mathrm{FEL} \mathrm{\&} 1866 \mathrm{ft} \text { FNL of Lse., } 2 \text { mi SE fr True } \\
\end{array}$ & & 3164 & 9/15/1953 & 9/21/1953 & coal \& Sh, sdy: 2867 \\
\hline 544 & $\begin{array}{l}\text { L.T. Burns, H.L. Leberman Jr. \#2 (oil prod) (Young County field) } \\
\text { TE\&\&L Co. Sur. A-457, Blk 210, } 432 \mathrm{ft} \text { \& \& } 1562 \mathrm{ft} \text { W of NE Corner Suv. \#210, } 750 \mathrm{ft} \text { of Well \#1 }\end{array}$ & & 889 & $5 / 17 / 1941$ & $5 / 27 / 1941$ & coal: 475 \\
\hline 545 & $\begin{array}{l}\text { L.T. Burns, H.L. Leberman Jr. \#3 (dry hole) } \\
\text { TE\&L Co. Sur. Blk } 210,432 \mathrm{ft} S \& 1862 \mathrm{ft} W \text { of NE Corner Suv. \#210, or } 300 \mathrm{ft} W \text { of } \# 2 \text { well } \\
\text { (Gunsight lime at } 825.5 \mathrm{ft} \text { ) }\end{array}$ & & 952 & 5/28/1941 & $5 / 31 / 1941$ & Sh, blk: 825 \\
\hline 546 & $\begin{array}{l}\text { L.T. Burns, H.L. Leberman Jr. \#4 (0il prod) (Young County field) } \\
\text { TE\&\&L Co. Sur. A-457, Blk } 210,432 \mathrm{ft} \text { \& \& } 1262 \mathrm{ft} \text { W of NE Corner Suv. \#210 (Gunsight lime at } 831 \mathrm{ft} \text { ) }\end{array}$ & & 889 & $5 / 31 / 1941$ & $6 / 3 / 1941$ & $\begin{array}{c}\text { coal: } 479 \\
\text { Sh, blk: } 830\end{array}$ \\
\hline 547 & $\begin{array}{l}\text { L.T. Burns, D. Logan \#1 (oil prod) (Burns-Ickert field) } \\
\text { I. Holman Crawford Past. Subd. Blk } 18 \text { (W } 1 / 2 \text { ) } 467 \mathrm{ft} \mathrm{FWL} \mathrm{\&} 410 \mathrm{ft} \text { FNL W } 1 / 2 \text { Blk } 18\end{array}$ & 1259 & 4650 & $6 / 3 / 1944$ & $7 / 11 / 1944$ & coal \& Sh stks: 890 \\
\hline 548 & $\begin{array}{l}\text { L.T. Burns, R.J. McCloud \#1 (dry hole) } \\
\text { TE\&L Co. Sur. Abst. } 85 \text { ? Sec. } 1395,700 \mathrm{ft} \text { E \& } 300 \mathrm{ft} \text { N of SW Cor. N } 30 \text { Ac. of } \\
\text { W } 60 \text { Ac. S } 160 \text { Ac. TE\&L Suv }\end{array}$ & & 927 & & & $\begin{array}{l}\text { Sh, dark: } 314 \\
\text { Sh, blk: } 430 \\
\text { Sh, blk: } 508 \\
\text { Sh, dark: } 580 \\
\text { Sh, dark: } 820 \\
\text { Sh, blk: } 825 \\
\text { Sh, dark: } 856\end{array}$ \\
\hline 549 & $\begin{array}{l}\text { L.T. Burns, S.A. Morris \#3 (dry hole) (Burns Ragland } 3200 \text { Strawn field) } \\
\text { TE\&L Co. Sur. Blk 258, Abst 505, } 160 \text { Acres, } 330 \mathrm{ft} \text { FEL \& } 330 \mathrm{ft} \text { FNL }\end{array}$ & & 3163 & 11/26/1953 & 12/1/1953 & Sh, blk: 1160 \\
\hline 550 & $\begin{array}{l}\text { L.T. Burns, G.M. Morrison \#1 (oil prod) (Burns Ragland field) } \\
\text { TE\&L Co. Sur. Blk 246, Sec. A-493, } 1400 \mathrm{ft} \text { \& \& } 467 \mathrm{ft} \text { of SW Cor. Suv. \#246, Abst. } 493\end{array}$ & & 4031 & 9/18/1941 & $\begin{array}{l}10 / 9 / 1941 \\
\end{array}$ & coal: 225 \\
\hline 551 & $\begin{array}{l}\text { L.T. Burns, G.M. Morrison \#3 (dry hole) (Burns Ragland Strawn field) } \\
\text { TE\&LL C. S.r. Blk 256, } 160 \text { Ac., } 330 \mathrm{ft} W \text { \& } 330 \mathrm{ft} \text { of SE Cor. of Suv. \#246, Abst. \#493, } \\
2.5 \mathrm{mi} \mathrm{NE} \text { fr True }\end{array}$ & & & & & $\begin{array}{l}\text { coal \& Sh: } 129 \\
\text { coaal: } 274 \\
\text { Sh, blk: } 1184 \\
\text { Sh, blk: } 1237\end{array}$ \\
\hline 552 & $\begin{array}{l}\text { L.T. Burns, H.M. Orr \#1 (dry hole) } \\
\text { TE\&L Suv. Bik } 631,160 \text { acres, } 150 \mathrm{ft} \text { fr N \& W lines of E } 1 / 2,160 \text { acres. }\end{array}$ & & 653 & $4 / 22 / 1932$ & 4/29/1932 & coal: 162 \\
\hline 553 & $\begin{array}{l}\text { Burns Oil Company, J.L. Rhodes \#1 (dry hole) } \\
\text { B.F. Kutch Sur. Abst. 133, } 505 \mathrm{ft} F S L \& \& 861 \mathrm{ft} \text { fr E \& W of } 40 \text { acres. }\end{array}$ & & 4123 & $11 / 7 / 1939$ & $1 / 6 / 1040$ & $\begin{array}{l}\text { Sh, blk: } 200 \\
\text { Sh, blk: } 484\end{array}$ \\
\hline 554 & $\begin{array}{l}\text { L.T. Burns, C.H. Rogers \#1 (oil prod) (Burns Ragland Miss. Lime field) } \\
\text { TE\&L Co. Sur. \#290, Abst. } 537,495 \mathrm{ft} N \& 495 \mathrm{ft} \text { of SW Cor. of N } 50 \text { Ac. Suv. \#290, being } 89 \mathrm{ft} \text { \& \& } \\
495 \mathrm{ft} \text { E of NW Cor. Suv. }\end{array}$ & & 4881 & $2 / 13 / 1943$ & 3/22/1943 & coal: 1232 \\
\hline 555 & $\begin{array}{l}\text { L.T. Burns, C.H. Rogers \# (dry hole) (Burns Ragland Strawn field) } \\
\text { TE\&LL Co. Sur. \#290, Sec. A-537, } 199 \text { acres, } 330 \mathrm{ft} F \mathrm{FL}, 660 \mathrm{ft} \text { SE of \#1, } 2.5 \mathrm{mi} \text { NE ff True }\end{array}$ & & 3006 & 4/14/1949 & 4/25/1949 & $\begin{array}{c}\text { coal: } 117 \\
\text { coal: } 1232\end{array}$ \\
\hline 556 & $\begin{array}{l}\text { L.T. Burns, W.T. Rogers \#12 (dry hole) (Rogers Pool field) } \\
\text { TE\&L Co. Sur. A-466, Sec. } 219,750 \mathrm{ft} E \& 1350 \mathrm{ft} \text { S of NW Corner of Ise., } 1.5 \mathrm{mi} \text { SE fr Olney } \\
\text { (Gunsight lime at } 860.5 \mathrm{ft} \text { ) }\end{array}$ & & 917 & 4/21/1941 & 4/26/1941 & coal: 482 \\
\hline 557 & $\begin{array}{l}\text { L.T. Burns, W.A. Rushing \#1 (oil prod) } \\
\text { TE\&L Co. Sur. Blk } 3401,1100 \mathrm{ft} N \& 1000 \mathrm{ft} \text { Eof the SW Cor of lease (lime GS at } 901 \mathrm{ft} \text { ) }\end{array}$ & & 903.5 & $2 / 23 / 1936$ & 3/8/1936 & $\begin{array}{l}\text { Sh, blk: } 458 \\
\text { Sh, blk: } 469 \\
\text { coal: } 546 \\
\text { Sh, blk: } 896\end{array}$ \\
\hline 558 & $\begin{array}{l}\text { L.T. Burns, W.A. Rushing \#2 (oil prod) } \\
\text { TE\&LL Co. Sur. Blk } 3401,320 \mathrm{ft} \text { E \& } 162 \mathrm{ft} \text { S of Well \#1 (lime GS at } 891 \mathrm{ft} \text { ) }\end{array}$ & & & & & $\begin{array}{l}\text { Sh, dark: } 550 \\
\text { Sh, blk: } 886\end{array}$ \\
\hline 559 & $\begin{array}{l}\text { L.T. Burns, W.A. Rushing \#3 (oil prod) } \\
\text { TE\&L Co. Suv. Sec. Fraction B, Blk } 3401,914 \mathrm{ft} \text { E \& } 813 \mathrm{ft} \text { Nof most southerly SW Cor. TE\&L Co. } \\
\text { Suv. } 3401 \text { (lime GS at } 897 \mathrm{ft} \text { ) }\end{array}$ & & 907 & $8 / 2 / 1936$ & 8/13/1936 & $\begin{array}{c}\text { Sh, dark: } 300 \\
\text { Sh, blk \& coal: } 474 \\
\text { Sh, blk: } 480 \\
\text { coal: } 484 \\
\text { Sh, blk: } 544 \\
\text { coal \& Sh, blk: } 550 \\
\text { Sh, blk: } 890 \\
\text { Sh, blk: } 892.5\end{array}$ \\
\hline 560 & $\begin{array}{l}\text { L.T. Burns, W.A. Rushing \#4 (oil prod) } \\
\text { TE\&L Co. Suv. Blk } 3401,614 \mathrm{ft} E \& 951 \mathrm{ft} \text { Nof most southerly SW Cor. of Suv. 3401, Fraction B } \\
\text { (lime GS at } 896 \mathrm{ft} \text { ) }\end{array}$ & & 906 & 8/16/1936 & 8/26/1936 & $\begin{array}{c}\text { Sh, blk: } 463 \\
\text { Sh, blk: } 473 \\
\text { coal: } 474 \\
\text { coal: } 560 \\
\text { Sh, blk: } 890 \\
\end{array}$ \\
\hline 561 & $\begin{array}{l}\text { L.T. Burns, W.A. Rushing } \$ 5 \text { (oil prod) } \\
\text { TE\&L Co. Suv. Bll } 3401,651 \mathrm{ft} \mathrm{N} \& 614 \mathrm{ft} \text { E of most southerly SW Cor. of Suv. 3401, Frac. B } \\
\text { (lime GS at } 899.5 \mathrm{ft} \text { ) }\end{array}$ & & 908.5 & $9 / 3 / 1936$ & $9 / 15 / 1936$ & $\begin{array}{c}\text { Sh, blk: } 473 \\
\text { Sh, blk: } 480 \\
\text { coal: } 484 \\
\text { Sh, blk: } 550 \\
\text { coal: } 558 \\
\text { Sh, blk: } 898 \\
\end{array}$ \\
\hline 562 & $\begin{array}{l}\text { L.T. Burns, W.A. Rushing \#6 (oil prod) } \\
\text { TE\&L Co. Suv. Blk } 3401,1214 \mathrm{ft} \text { E \& } 651 \mathrm{ft} \text { of most southerly SW Cor. Suv. } 3401 \text {, Fraction B, } \\
\text { or } 301 \mathrm{ft} \text { (?) of Well \#2 (lime G.S.Sat } 902 \mathrm{ft} \text { ) }\end{array}$ & & 917 & 9/14/1936 & $10 / 2 / 1936$ & $\begin{array}{c}\text { Sh, blk: } 486 \\
\text { Sh, blk: } 500 \\
\text { Sh, blk \& coal: } 564 \\
\text { Sh, dark: } 894 \\
\text { Sh, blk: } 896\end{array}$ \\
\hline 563 & $\begin{array}{l}\text { L.T. Burns, W.A. Rushing \#7 (oil prod) } \\
\text { TE\&L Co. Suv. Blk } 3401, \text { Fract. B, } 614 \mathrm{ft} \text { E \& } 1251 \mathrm{ft} \mathrm{N} \text { of most southerly SW Cor. of Suv. } 3401 \text {, } \\
300 \mathrm{ft} N \# 4 \text { (lime hd Gsat } 897 \mathrm{ft} \text { ) }\end{array}$ & & 906 & $10 / 6 / 1936$ & 10/17/1936 & $\begin{array}{l}\text { Sh, blk: } 290 \\
\text { Sh, blk: } 468 \\
\text { Sh, blk \& coal: } 481 \\
\text { Sh, blk: } 512 \\
\text { coal: } 550 \\
\text { Sh, blk \& coal: } 560 \\
\text { Sh, dark: } 635 \\
\text { Sh, blk: } 775 \\
\text { Sh, blk: } 890 \\
\end{array}$ \\
\hline 564 & $\begin{array}{l}\text { L.T. Burns, W.A. Rushing \#8 (oil prod) } \\
\text { TE\&L Co. Suv. Blk } 3401,914 \mathrm{ft} E \& 1413 \mathrm{ft} \mathrm{N} \text { of most southerly SW Cor. Suv. } 3401 \text {, Frac. B, } \\
\text { or } 300 \mathrm{ft} N \text { Well \#1 (lime hd GS at } 896 \mathrm{ft} \text { ) }\end{array}$ & & 906.5 & 10/19/1936 & 10/31/1936 & $\begin{array}{l}\text { Sh, blk \& coal: } 468 \\
\text { Sh, blk: } 477 \\
\text { caal: } 478 \\
\text { caal: } 550 \\
\text { Sh, blk: } 660 \\
\text { Sh, blk: } 889\end{array}$ \\
\hline 565 & $\begin{array}{l}\text { L.T. Burns, W.A. Rushing \#10 (oil prod) } \\
\text { TE\&L Co. Suv. Blk } 3401 \text {, Frac. B, } 314 \mathrm{ft} \text { E \& } 1101 \mathrm{ft} \text { N of most southerly SW Cor. of Suv. } 3401 \\
\text { (lime G.S. at } 896 \mathrm{ft} \text { ) }\end{array}$ & & 905.5 & $5 / 29 / 1937$ & $6 / 8 / 1937$ & $\begin{array}{l}\text { Sh, dark: } 280 \\
\text { Sh, blk: } 284 \\
\text { Sh, blk: } 460 \\
\text { Sh, blk: } 470 \\
\text { Sh, dark: } 628 \\
\text { Sh, blk: } 720 \\
\text { Sh, blue \& dark: } 725 \\
\text { Sh, blk: } 890\end{array}$ \\
\hline
\end{tabular}




\begin{tabular}{|c|c|c|c|c|c|c|}
\hline $\begin{array}{l}\text { Occurrence } \\
\text { No. }\end{array}$ & $\begin{array}{l}\text { Well name / } \\
\text { location }\end{array}$ & $\begin{array}{c}\text { Elevation } \\
\text { (ft) }\end{array}$ & $\begin{array}{c}\text { Total } \\
\text { depth (ft) }\end{array}$ & $\begin{array}{l}\text { Spud } \\
\text { date }\end{array}$ & $\begin{array}{c}\text { Compl; PB } \\
\text { dates }\end{array}$ & $\begin{array}{l}\text { Coal and black or dark-gray shale } \\
\text { occurrences (depth in ft) }\end{array}$ \\
\hline 566 & $\begin{array}{l}\text { L.T. Burns, W.A. Rushing \#11 (oil prod) } \\
\text { TE\&L Co. Suv. Blk } 3401 \text {, Sec. Fr-B, } 1214 \mathrm{ft} \text { E \& } 1251 \mathrm{ft} \text { Nof most southerly SW Cor. Suv. } 3401 \text {, } \\
300 \mathrm{ft} \text { Nof \#2 (lime G.S. at } 895 \mathrm{ft} \text { ) }\end{array}$ & & 902 & 6/14/1937 & 6/25/1937 & $\begin{array}{l}\text { coal: } 461 \\
\text { Sh, blk: } 538 \\
\text { Sh, dark: } 547 \\
\text { Sh, blk: } 890 \\
\end{array}$ \\
\hline 567 & $\begin{array}{l}\text { L.T. Burns, L. Williams \#1 (oil prod) (Young County Burns Ragland field) } \\
\text { TE\&\&L Co. \#257, } 467 \mathrm{ft} S \& 1436 \mathrm{ft} \text { W of NE Cor. of TE\&L Suv. \#257, } 2.5 \mathrm{mi} \text { N \& Efr True }\end{array}$ & & 5014 & 10/21/1941 & 7/27/1942 & $\begin{array}{l}\text { coal: }: 317 \\
\text { coal \& Sh, blk: } 1183 \\
\text { coal: } 1211\end{array}$ \\
\hline 568 & $\begin{array}{l}\text { L.T. Burns, L. Williams \#2 (oil prod) (Burns Ragland-Mississippi Lime field) } \\
\text { TE\&L Co. Blk 257, Ab. 504, } 1400 \mathrm{ft} \text { \& \& } 467 \mathrm{ft} \text { W of NE Cor. of TE\&L Suv. 257, Ab. } 504\end{array}$ & 1187 & 4895 & 4/19/1944 & 5/17/1944 & $\begin{array}{c}\text { coal: } 251 \\
\text { coal: } 1208\end{array}$ \\
\hline 569 & 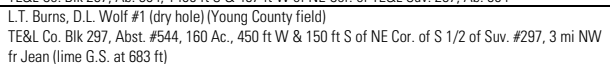 & & 750 & $2 / 4 / 1948$ & $2 / 11 / 1948$ & $\begin{array}{l}\text { Sh, blk: } 456 \\
\text { Sh, blk: } 682\end{array}$ \\
\hline 560 & $\begin{array}{l}\text { L.T. Burns \& E.M. Stilley, J.D. Spencer \#1 (dry hole) } \\
\text { TE\&L Co. Suvv., Blk 1396, Abst. } 854,1869 \mathrm{ft} \text { W } 150 \mathrm{ft} \text { Nof SE Cor. of N } 160 \text { Ac. (lime G.S. at } 795 \mathrm{ft} \text { ) }\end{array}$ & & 864 & 6/20/1936 & 6/29/1936 & $\begin{array}{l}\text { Sh, blk: } 214 \\
\text { Sh, blk: } 371 \\
\text { Sh, blk: } 788\end{array}$ \\
\hline 561 & $\begin{array}{l}\text { P.C. Burns, A.A. Bernheart \#1 (dry hole) (WC Young County field) } \\
\text { TE\&L Co. Suv, Blk } 238,150 \mathrm{ft} \text { N \& W lines S } 40 \text { Ac. of E } 80 \text { Ac. of S. } 160 \text { Ac. of Blk } 238 \\
\text { (G.S. Lime at } 987 \mathrm{ft})\end{array}$ & 1218 & 1068 & 6/24/1941 & 6/27/1941 & Sh, dark: 986 \\
\hline 562 & $\begin{array}{l}\text { P.C. Burns, J.M. Bloodworth \#1 (dry hole) } \\
\text { TE\&L Co. Suv., Blk 210, } 50 \text { Ac., } 150 \mathrm{ft} \text { \& \& E lines S } 50 \text { Ac. Tr., } 150 \mathrm{ft} \text { fr S \& E lines, } 4 \text { mi S fr Olney } \\
\text { (G.S. lime at } 820 \mathrm{ft} \text { ) }\end{array}$ & 1166 & 871 & $7 / 12 / 1941$ & 7/16/1941 & $\begin{array}{c}\text { Coal: } 467 \\
\text { Sh, blk: } 819\end{array}$ \\
\hline 563 & $\begin{array}{l}\text { P.C. Burns, R. Campbell \#4 (oil prod) (North Young Dist. 9) } \\
\text { TE\&L Co. Suv., Blk 231, Abst. \#478, } 450 \mathrm{ft} \text { S \& } 150 \mathrm{ft} \text { W of NE Corner Blk \#231, Abst. } 478\end{array}$ & & 1000 & 9/28/1940 & 10/1/1940 & coal: 620 \\
\hline 564 & $\begin{array}{l}\text { P.C. Burns, F. Clark \#1 (dry hole) (Young County field) } \\
\text { TE\&L Co. Suv. Blk } 218,40 \text { Ac. } 1050 \mathrm{ft} \text { SL \& } 150 \mathrm{ft} \mathrm{WL.} 3 \mathrm{mi} \text { SE fr Olney (G.S. lime at } 887 \mathrm{ft} \text { ) }\end{array}$ & & 929 & $5 / 7 / 1941$ & $5 / 10 / 1941$ & $\begin{array}{l}\text { Sh \& coal: } 439 \\
\text { Sh, blk: } 886.5\end{array}$ \\
\hline 565 & $\begin{array}{l}\text { P.C. Burns, E.J. Larimore \#10 (oil prod) (Young County Regular field) } \\
\text { TE\&L Co. Suv., Blk 210, } 196 \text { Ac., } 850 \mathrm{ft} \text { FNL \& } 2400 \mathrm{FEL} \text { of } 196 \mathrm{Ac} \text {. (G. S. lime at } 833 \mathrm{ft})\end{array}$ & & 885 & $4 / 30 / 1960$ & $5 / 1 / 1960$ & Sh \& coal: 405 \\
\hline 566 & $\begin{array}{l}\text { Burris \& Jackson Drilling Co., D.L. Bishop \#1 (dry hole) (Young County field) } \\
\text { TE\&L Co. Suv., Bik } 1593,92.53 \text { ac., } 150 \mathrm{ft} \mathrm{FEL} \mathrm{\&} 150 \mathrm{ft} \text { FNL of Lse., } 7 \text { mi W fr Olney } \\
\text { (G. S. lime at } 833 \mathrm{ft} \text { ) }\end{array}$ & & 1225 & $8 / 4 / 1953$ & 8/6/1953 & $\begin{array}{l}\text { Sh, sdy; coal: } 810 \\
\text { Sh; coal: } 840 \\
\text { Sh, sdy; coal: } 922 \\
\text { Sh, blk: } 1217 \\
\end{array}$ \\
\hline 567 & $\begin{array}{l}\text { Burris \& Jackson Drilling Co., Gadberry \#1 (dry hole) (Young County Regular field) } \\
\text { TE\&L Co. Suv., Blk } 227,79 \text { ac., } 450 \mathrm{ft} \text { FEL \& } 450 \mathrm{ft} \text { FSL of Lse., } 79 \text { ac., } 3 \text { mi SE fr Olney } \\
\text { (G S lime at } 962 \mathrm{ft} \text { ) }\end{array}$ & & 981 & 8/18/1954 & 8/20/1954 & $\begin{array}{l}\text { Sh; coal: } 512 \\
\text { Sh; coal: } 690 \\
\text { Sh, blk: } 954 \\
\text { Sh, dk gray: } 960\end{array}$ \\
\hline 568 & $\begin{array}{l}\text { Burris \& Jackson, E. McClatchy \#1 (dry hole) (Young County field) } \\
\text { BBB\&C, Abst. 419, BI 172, } 40 \text { Ac., } 450 \mathrm{ft} \text { FNL \& FWL of } 40 \text { Ac., } 2 \text { mi SW of S of Olney }\end{array}$ & & 1111 & 3/28/1956 & 3/30/1956 & $\begin{array}{l}\text { Sh \& coal: } 471 \\
\text { Sh \& coal stks: } 800 \\
\text { Sh, dk gray: } 920 \\
\text { Sh, blk: } 1100\end{array}$ \\
\hline 569 & $\begin{array}{l}\text { Burris \& Jackson, R.C. Self \#1 (dry hole) (Young County field) } \\
\text { TE\&L Co. Suv., Blk 75, } 160 \text { Ac., } 462 \mathrm{ft} f \mathrm{fr} \& \& \text { EL of SE/4 of SW/4 of Blk } 75,6 \mathrm{mi} \text { SW of Olney }\end{array}$ & & 1095 & $7 / 27 / 1955$ & $7 / 28 / 1955$ & $\begin{array}{l}\text { Sh \& \& \& coal: } 736 \\
\text { Sh, gy \& coal stks: } 795 \\
\text { coal \& Sh: } 891 \\
\text { Sh blk: } 1067\end{array}$ \\
\hline 570 & $\begin{array}{l}\text { R.H. Busby et al., Cusenmary (Cuisenbery) \#1 (1) (oil prod) (WC); Young County Sch. } \\
\text { Land Sur., Blk 18, Sec. A-1285, } 160 \text { Ac., } 1030 \mathrm{ft} \text { Nof SL \& } 330 \mathrm{ft} \text { W of EL, in Murray }\end{array}$ & & 3664 & 6/9/1955 & $6 / 201955$ & $\begin{array}{c}\text { conal: } 191 \\
\end{array}$ \\
\hline 571 & $\begin{array}{l}\text { R.H. Busby et al., J. Latimer \#1 (dry hole) (Murray field) } \\
\text { Young County Sch. Land Sur., Blk 17, Sec. A-1285, } 136.5 \text { Ac., } 467 \mathrm{ft} \text { FWL \& } 1263 \mathrm{ft} \text { FNL of lease }\end{array}$ & & 3678 & 8/9/1954 & 8/24/1954 & coal; Sh: 236 \\
\hline 572 & $\begin{array}{l}\text { G.T. Buskirk, F.U. Powell \#1 * *dry hole) (WC) } \\
\text { TE\&L Blk } 1586,80 \text { Ac., } 250 \mathrm{ft} \text { FNL \& } 150 \mathrm{ft} F \mathrm{FL}, 4.5 \mathrm{mi} \text { S \& } 0.5 \mathrm{mi} \text { E of Megargel ( } G \text { S lime at } 1348 \mathrm{ft} \text { ) }\end{array}$ & & 1350 & 4/7/1956 & $4 / 9 / 1956$ & $\begin{array}{c}\text { coal: } 932 \\
\text { Sh, blk: } 1344\end{array}$ \\
\hline 573 & $\begin{array}{l}\text { F.E. Butler, J.L. Boone \#1 (dry hole) (N Young field) } \\
\text { BBB\&C Suv., } 80 \text { Ac., NE } 1 / 4 \text { of Tr., distance from lines } n \text { given, } 3 \text { mi N fr Loving }\end{array}$ & & 532 & 12/9/1941 & 12/22/1941 & $\begin{array}{c}\text { Sh, boli: } 1344 \\
\text { coal: } 152\end{array}$ \\
\hline 574 & $\begin{array}{l}\text { F.E. Butler et al., W.H. Clarida \#1 (dry hole) } \\
\text { L.E. Hardin Suv., Abst \#1682, well located in NW Corner of } 40 \text { Ac }\end{array}$ & & 645 & $11 / 19 / 1933$ & $12 / 10 / 1933$ & $\begin{array}{l}\text { coal: } 170 \\
\text { coal: } 225 \\
\text { Sh, blk: } 580\end{array}$ \\
\hline 575 & $\begin{array}{l}\text { F.E. Butler, L.R. Davis \#1 (dry hole) } \\
\text { G\&BN Co. \#4 Suv., } 80 \text { Acres, } 750 \text { ft FNL, } 150 \mathrm{ft} \text { FEL }\end{array}$ & & 804 & $3 / 30 / 1937$ & $5 / 22 / 1937$ & $\begin{array}{l}\text { Sh, blk: } 300 \\
\text { Sh, blk: } 378 \\
\text { Sh, blk: } 770 \\
\text { Sh. blk: } 780\end{array}$ \\
\hline 576 & $\begin{array}{l}\text { F.E. Butler et al., W.H. Dyer \#1 (dry hole) } \\
\text { L.E. Hardin Suv., } 150 \text { ft each way fr NE Cor. Of L.E. Hardin Survey }\end{array}$ & & 632 & 8/2/1934 & $8 / 19 / 1934$ & $\begin{array}{l}\text { Sh, blk \& brn: } 130 \\
\text { Sh, bl: } 215 \\
\text { Sh, blk: } 560\end{array}$ \\
\hline 577 & $\begin{array}{l}\text { F.E. Butler, R. C. Howard \#1 (dry hole) } \\
\text { TE\&L Co. Suv., Blk } 243,150 \mathrm{ft} \text { each way fr SE Cor. N } 160 \text { Ac. Blk } 243\end{array}$ & & 800 & $8 / 27 / 1931$ & 9/6/1931 & $\begin{array}{l}\text { Sh, blk: } 110 \\
\text { coli: } 260 \\
\text { Sh, blk: } 290 \\
\text { Sh, blk: } 342\end{array}$ \\
\hline 578 & $\begin{array}{l}\text { F.E. Butler et al., C.W. Johnson Estate \#1 (dry hole) } \\
\text { E.W. Sergeant Suv. Abst. } 253,150 \mathrm{ft} \text { FEL \& } 450 \mathrm{ft} \text { FNL of Middle } 60 \text { Ac of E } 120 \text { Ac. S } 200 \text { Ac. }\end{array}$ & & 555 & 4/14/1936 & 4/24/1936 & $\begin{array}{l}\text { coal: } 100 \\
\text { Sh, blk: } 467\end{array}$ \\
\hline 579 & $\begin{array}{l}\text { F.E. Butler, R. E. Jones \#1 (dry hole) } \\
\text { Susan Salle Suv. Blk 252, } 40 \text { Ac., } 450 \mathrm{ft} \mathrm{FSL} \mathrm{\&} 450 \mathrm{ft} \text { FWL } 40 \text { Ac. }\end{array}$ & & 631 & $8 / 23 / 1933$ & 9/1/1933 & $\begin{array}{c}\text { coal: } 30 \\
\text { Sh, blk: } 205\end{array}$ \\
\hline 580 & $\begin{array}{l}\text { F.E. Butler, W.R. Ledbetter \#1 (dry hole) } \\
\text { Sylphia Tynes Suv., Abst. } 274,40 \text { Acre, } 150 \mathrm{ft} \text { FWL \& } 200 \mathrm{ft} F \mathrm{FL}\end{array}$ & & 600 & 2/20/1935 & $3 / 8 / 1935$ & $\begin{array}{c}\text { coal: } 85 \\
\text { Sh, blk: } 425\end{array}$ \\
\hline 581 & $\begin{array}{l}\text { F.E. Butler et al., E. Bernhardt \#f (dry hole) } \\
\text { TE\&L Co. B Blk 238, } 150 \mathrm{ft} \text { fr N \& W lines, } 40 \text { Ac. Tr., NE } 1 / 4 \text { Sec. }\end{array}$ & & 1042 & $7 / 13 / 1936$ & $8 / 27 / 1936$ & $\begin{array}{l}\text { Sh, blk: } 590 \\
\text { Sh, blk: } 680\end{array}$ \\
\hline 582 & $\begin{array}{l}\text { Butter et al., W.R. Farmer et al. } 1 \text {. (dry hole) } \\
\text { John Anderson Suv., } 150 \mathrm{ft} \text { E of center of WL }\end{array}$ & & 1102 & $12 / 11 / 1929$ & $1 / 5 / 1930$ & Sh, blk: 1000 \\
\hline 583 & $\begin{array}{l}\text { F.E. Butler, R.O. Prideaux \#1 (dry hole) } \\
\text { A. McMullen Suv., Blk } 198,196 \mathrm{ft} \mathrm{FNL} \mathrm{\&} 150 \mathrm{ft} F \mathrm{FE}, 20 \mathrm{Ac} \text {. }\end{array}$ & & 800 & $8 / 18 / 1938$ & 9/7/1938 & $\begin{array}{l}\text { Sh, blk: } 335 \\
\text { Sh, blk: } 426 \\
\text { [Sh?), blk: } 515 \\
\text { (Sh?), blk: } 748\end{array}$ \\
\hline 584 & $\begin{array}{l}\text { Butler Calhoun \& Soyton, S.M.J. Benson Estate \#2 (oil prod) } \\
\text { TE\&L Co. Suv, Blk 1398, } 150 \mathrm{ft} \text { fr S \& E lines, S } 40 \text { Ac., W } 100 \text { Ac., E } 200 \text { Ac. S } 250 \text { Ac. of } 320 \text { Ac. Tr. }\end{array}$ & & 937 & 5/15/1938 & $5 / 30 / 1938$ & $\begin{array}{l}\text { Sh, blk: } 472 \\
\text { Sh, blk: } 552 \\
\text { Sh, blk: } 892\end{array}$ \\
\hline 585 & $\begin{array}{l}\text { Butler \& McAlister, J.K. Thomas \#1 (oil prod) } \\
\text { TE\&L Co. Survey, } 150 \mathrm{ft} \text { fr } \& \& \text { W lines }\end{array}$ & 1201 & 1002 & $3 / 26 / 1929$ & $4 / 13 / 1929$ & $\begin{array}{l}\text { Sh, dark: } 540 \\
\text { Sh, blk: } 575 \\
\text { Slate, blk: } 660\end{array}$ \\
\hline 586 & $\begin{array}{l}\text { Buttram \& Newby, Ferguson \#1 (dry hole) } \\
\text { L.W. Smith Suv., } 160 \text { Ac., } 768 \text { ft FNL \& } 150 \text { ft FEL, } 3 \text { mi S fr Markley }\end{array}$ & & 590 & $7 / 1 / 1941$ & $7 / 20 / 1941$ & 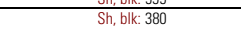 \\
\hline 587 & $\begin{array}{l}\text { Buttram \& Newby, Ferguson \#1 (dry hole)(WC) } \\
\text { S. Thynes Suv., } 209 \text { Acres, } 622 \mathrm{ft} \mathrm{S}, 1125 \mathrm{ft} \text { West line, } 3 \mathrm{mi} \text { S fr Markley }\end{array}$ & & 563 & $6 / 13 / 1941$ & $6 / 25 / 1941$ & $\begin{array}{l}\text { Sh, blk: } 40 \\
\text { Sh, blk: } 385\end{array}$ \\
\hline 588 & $\begin{array}{l}\text { C.C. Butts, R.E. Daily \#1 (Young County Regular field) } \\
\text { TE\&L Co. Survey, Blk } 167,150 \mathrm{ft} F E L \text { \& } 450 \mathrm{ft} F \mathrm{FL}\end{array}$ & & 902 & $11 / 3 / 1949$ & $11 / 4 / 1949$ & $\begin{array}{l}\text { coal \& slate: } 328 \\
\text { coal: } 552\end{array}$ \\
\hline 589 & 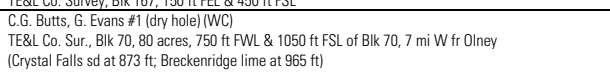 & & 1308 & $5 / 18 / 1950$ & $5 / 20 / 1950$ & $\begin{array}{l}\text { Sh \& coal: } 850 \\
\text { coal \& slate: } 886 \\
\text { Sh, blk \& lime shell: } 1260\end{array}$ \\
\hline 590 & $\begin{array}{l}\text { C.G. Butts, G. Rice \#2 (dry hole) (Young County Area field) } \\
\text { I. Holman Sur., Sec. A-130, } 20 \text { acres, } 750 \mathrm{ft} \text { FWL \& } 150 \mathrm{ft} \text { FNL of Ise. } 7 \mathrm{mi} \text { W fr Olney }\end{array}$ & 1267 & 1296 & $3 / 8 / 1949$ & $3 / 10 / 1949$ & Sh, blk: 1251 \\
\hline 591 & $\begin{array}{l}\text { C.G. Butts, L. Smith \#1 (dry hole) (WC) } \\
\text { TE\&L Co. Sur., Blk 1365, } 40 \text { acres, } 150 \mathrm{ft} f \mathrm{fr} \text { \& E lines of Ise., } 7 \mathrm{mi} \text { SE fr Olney (b.s. lime at } 832 \mathrm{ft} \text { ) }\end{array}$ & & 917 & $6 / 20 / 1950$ & $6 / 22 / 1950$ & $\begin{array}{l}\text { coal: } 434 \\
\text { Sh \& coal: } 476 \\
\text { coal: } 480 \\
\text { Sh bl: } 831\end{array}$ \\
\hline 592 & $\begin{array}{l}\text { C.G. Butts \& L. Mitchell, D.E. Logan Estate \#3 (dry hole) (Young County Regular field) } \\
\text { I. Holman Blk } 18,4875 \text { ac. } 450 \mathrm{ft} F \mathrm{~S} \& \text { WL of W/2 of Blk } 18,5 \mathrm{mi} \text { SE of Megargel }\end{array}$ & & 1262 & $12 / 5 / 1956$ & $12 / 6 / 1956$ & $\begin{array}{l}\text { Sh, blk: } 831 \\
\text { Sh, blk: } 1226\end{array}$ \\
\hline 593 & $\begin{array}{l}\text { C.G. Butts \& C.V. Morgan, M.U. Howard \#1 (dry hole (Young County North field); TE\&L Co. } \\
\text { Sur., Blk 1459, Sec. } 1459,54.8 \text { acres, } 450 \mathrm{ft} \text { ff S \& E Ins., } 6 \text { mi SE fr Olney (G S lime at } 784 \mathrm{ft} \text { ) }\end{array}$ & & 834 & $1 / 18 / 1950$ & $1 / 19 / 1950$ & Sh, blk: 782 \\
\hline 594 & 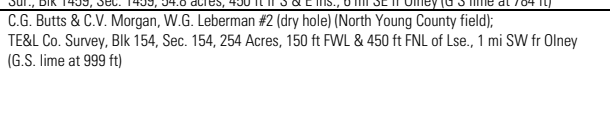 & 1246 & 1032 & 1/7/1951 & 1/8/1951 & $\begin{array}{c}\text { coal, Sh \&slate: } 356 \\
\text { coal: } 365 \\
\text { caal: } 386 \\
\text { caal: } 605 \\
\text { Sh, blk: } 998 \\
\text { Sh. blk \& coal: } 1016\end{array}$ \\
\hline
\end{tabular}




\begin{tabular}{|c|c|c|c|c|c|c|}
\hline $\begin{array}{c}\text { Occurrence } \\
\text { No. }\end{array}$ & $\begin{array}{l}\text { Well name / } \\
\text { location }\end{array}$ & $\begin{array}{c}\text { Elevation } \\
\text { (ft) }\end{array}$ & $\begin{array}{c}\text { Total } \\
\text { depth (ft) }\end{array}$ & $\begin{array}{l}\text { Spud } \\
\text { date }\end{array}$ & $\begin{array}{c}\text { Compl; PB } \\
\text { dates }\end{array}$ & $\begin{array}{l}\text { Coal and black or dark-gray shale } \\
\text { occurrences (depth in ft) }\end{array}$ \\
\hline 595 & $\begin{array}{l}\text { C.G. Butts \& C.V. Morgan, S.P. Ligon et al., \#1 (oil prod)(Young County Regular field) } \\
\text { TE\&L Suv., Block 1998, Sec. } 1998\end{array}$ & & 3599 & 3/1/1958 & 3/10/1958 & $\begin{array}{l}\text { Sh \& Sd with coal stks: } 440 \\
\text { Sh with coal \& lime stks: } 548\end{array}$ \\
\hline 596 & $\begin{array}{l}\text { J.D. Byron, E.A. Edwards \#1 (dry hole) } \\
\text { TE\&\&L Survey, Blk } 1499,74 \text { Acres, } 900 \mathrm{ft} \text { E of W \& } 150 \mathrm{ft} \mathrm{N} \text { of S lines, TE\&L Survey, Blk } 1499\end{array}$ & & 1001 & $5 / 11 / 1928$ & $6 / 20 / 1928$ & $\begin{array}{c}\text { Slate, blk: } 365 \\
\text { Slate, blk: } 798 \\
\text { Sh, blk: } 895\end{array}$ \\
\hline 597 & $\begin{array}{l}\text { J.D. Byron, J.W. Edwards \#1 (dry hole) } \\
\text { TE\&\& Survey, Blk } 1499,450 \mathrm{ft} \text { of } W \& 450 \mathrm{ft} S \text { of } \mathrm{N} \text { lines }\end{array}$ & 1188 & 867 & 4/3/1928 & $4 / 30 / 1928$ & $\begin{array}{c}\text { coal: } 409 \\
\text { Sh, blk: } 805\end{array}$ \\
\hline
\end{tabular}




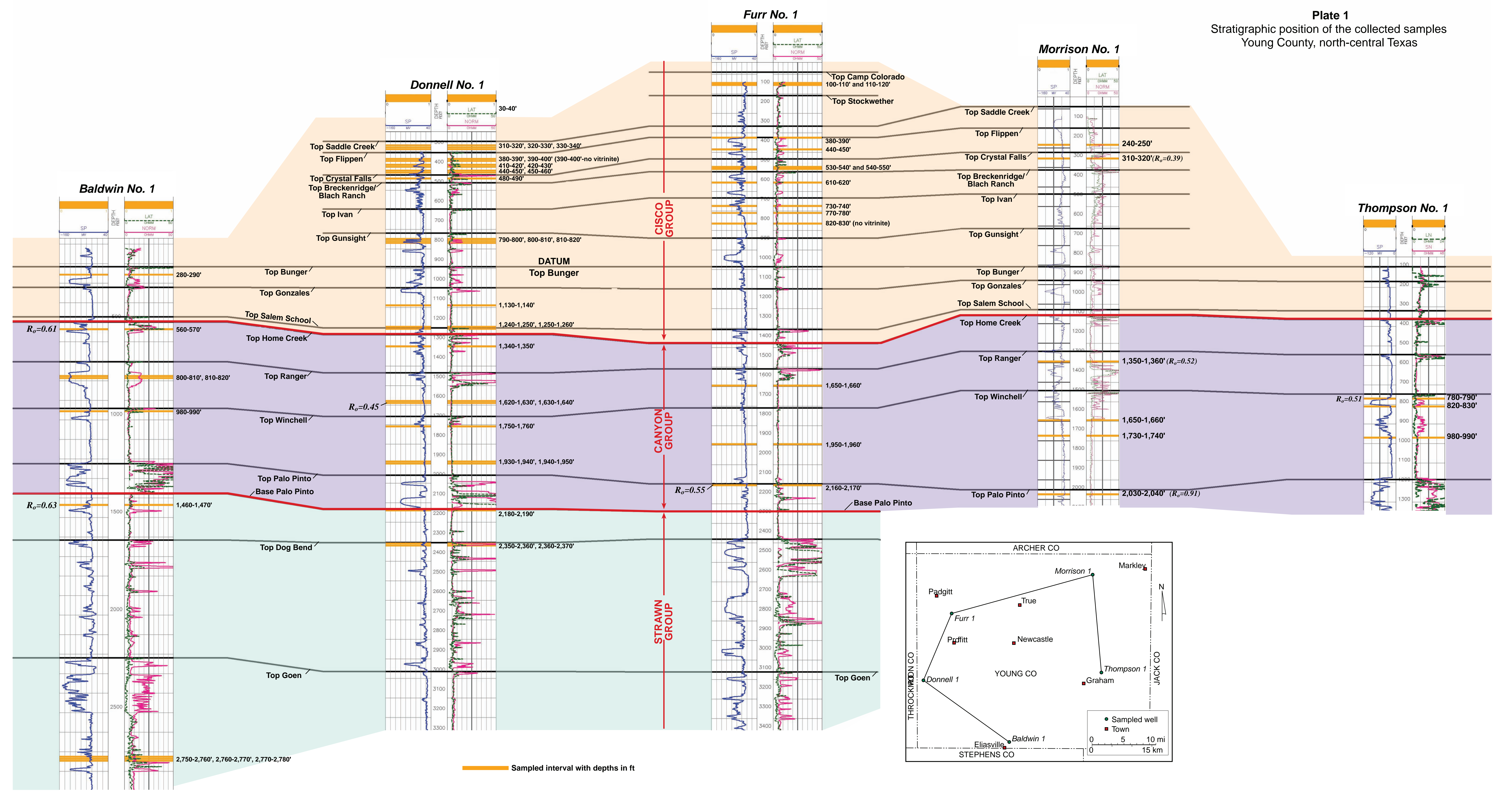

